\title{
Dissecting gating mechanisms of Orai calcium channel paralogs using constitutively active Orai mutants that mimic STIM1-gated state
}

Bartłomiej Augustynek ${ }^{1}$, Gergely Gyimesi ${ }^{1}$, Jan Dernič ${ }^{1}$, Matthias Sallinger ${ }^{2}$, Giuseppe Albano ${ }^{1}$, Gabriel J. Klesse ${ }^{1}$, Palanivel Kandasamy ${ }^{1}$, Herwig Grabmayr ${ }^{2}$, Irene Frischauf ${ }^{2}$, Daniel G. Fuster ${ }^{1}$, Christine Peinelt ${ }^{3}$, Matthias A. Hediger $^{1 *}$ and Rajesh Bhardwaj ${ }^{1,4 *}$

${ }^{1}$ Membrane Transport Discovery Lab, Department of Nephrology and Hypertension and Department of Biomedical Research, Inselspital, University of Bern, Freiburgstrasse 15, CH-3010 Bern, Switzerland.

${ }^{2}$ Institute of Biophysics, JKU Life Science Center, Johannes Kepler University Linz, A-4020 Linz, Austria.

${ }^{3}$ Institute of Biochemistry and Molecular Medicine, University of Bern, Bühlstrasse 28, 3012 Bern, Switzerland.

${ }^{4}$ Current address: Signal Transduction Laboratory, National Institute of Environmental Health Sciences, NIH, 111 TW Alexander Drive, NC 27709, USA.

*For correspondence: Matthias A. Hediger (matthias.hediger@ibmm.unibe.ch); Rajesh Bhardwaj (rajesh.bhardwaj@nih.gov) 
Augustynek et al.

Gating mechanisms of Orai calcium channels

\section{Abstract}

In humans, there are three paralogs of the Orai $\mathrm{Ca}^{2+}$ channel, which lie at the heart of the store-operated calcium entry (SOCE) machinery. While the STIM-mediated gating mechanism of Orai channels is still being actively investigated, several artificial and natural variants are known to cause constitutive activity of the human Orai1 channel. Surprisingly, little is known about the conservation of the gating mechanism among the different human Orai paralogs and orthologs in other species. In our work, we show that the mutation corresponding to the activating mutation H134A in transmembrane helix 2 (TM2) of human Orai1 also activates Orai2 and Orai3, likely via a similar mechanism. However, this cross-paralog conservation does not apply to the "ANSGA" nexus mutations in TM4 of human Orai1 which mimic the STIM1-activated state of the channel. Investigating the mechanistic background of these differences, we identified two positions, H171 and F246 in human Orai1, which directly control the channel activation triggered by the "ANSGA" mutations in Orai1. Our results shed new light on these important gating checkpoints and show that the gating mechanism of the Orai channels is affected by multiple factors that are not necessarily evolutionarily conserved, such as the TM4-TM3 coupling.

\section{Introduction}

Store-operated calcium entry (SOCE) is a ubiquitous mechanism by which non-excitable cells regulate basal cytosolic calcium levels and the replenishment of intracellular $\mathrm{Ca}^{2+}$ stores. This mechanism is critically important as calcium participates in many different signaling pathways that play central roles in a wide range of cellular processes such as cell division, growth, differentiation, metabolism, gene expression, immune function and others. The SOCE machinery is a multi-component system in which the key components known as Orai proteins are present in the plasma membrane, where they form the pores of the $\mathrm{Ca}^{2+}$ release-activated $\mathrm{Ca}^{2+}(\mathrm{CRAC})$ channels (Feske et al., 2006; Prakriya et al., 2006; Vig et al., 2006), and the $\mathrm{Ca}^{2+}$-sensing STIM proteins are anchored in the ER membrane, from where they regulate gating and activity of the Orai channels (Liou et al., 2005; Roos et al., 2005). In humans, there are three known Orai paralogs (Orai1-3; referred to as hO1, hO2 and hO3, respectively) and two STIM paralogs (STIM1 and STIM2). Furthermore, due to the tissue-specific mRNA splicing, several splice variants of these proteins have been identified (Berna-Erro, Jardin, Salido, \& Rosado, 2017; Darbellay, Arnaudeau, Bader, Konig, \& Bernheim, 2011; Fukushima, Tomita, Janoshazi, \& Putney, 2012; Knapp et al., 2020; Miederer et al., 2015; Niemeyer, 2016; Ramesh et al., 2021; Rana et al., 2015). 
Augustynek et al.

Gating mechanisms of Orai calcium channels

Several structural studies have shown that the CRAC channel pore comprises a hexameric arrangement of Orai proteins (Hou, Burstein, \& Long, 2018; Hou, Outhwaite, Pedi, \& Long, 2020; Hou, Pedi, Diver, \& Long, 2012; Liu et al., 2019). The Orai protein itself has four transmembrane (TM) helices, which are arranged radially around the central pore that is formed out of six TM1 helices, one from each subunit (Figure 1). Additional twelve TM helices (6x TM2 and 6x TM3) are arranged in a second, interwoven ring wrapping around the pore-forming TM1 helices. Finally, six TM4 helices form a third ring at the distal region (Hou et al., 2012). Interfaces between the four helices TM1-4 show tight packing of amino acid sidechains. The N-terminus of TM1 that extends into the cytoplasm with residues of unresolved structure encompasses a region (residues 39-59) dispensable for SOCE but critical for ensuring coupling of hO1-mediated local $\mathrm{Ca}^{2+}$ entry to the activation of NFAT1 (Nuclear Factor of Activated Tcells) transcription factor (Kar et al., 2021). Notably, the TM4 features a kink at a highly conserved transmembrane proline residue (Pro245 in h01), followed by another hinge region with the conserved cytosolic sequence of LVSHK (L261-K265 in h01) residues, also called the "nexus" region (Y. Zhou et al., 2016), and finally a C-terminal, cytosolic extension helix (TM4ext). The first structural studies based on Drosophila melanogaster Orai (dOrai) described TM4ext as being locked in a so-called "latched" state, nearly parallel to the membrane bilayer by coiled coil interactions of pairs of antiparallel TM4ext helices between neighboring Orai subunits (Hou et al., 2012). Whether the latched state is a true representation of the native quiescent state of the Orai 1 channel is contested and is discussed later including insights from new structural findings.

Mutations in several key regions of the Orai proteins have shown constitutive activity independent of STIM1 gating. These variants have been instrumental to understand the gating mechanism of Orai channels (Krizova, Maltan, \& Derler, 2019; Yeung, Yamashita, \& Prakriya, 2020). The hO1 pore-lining residues F99 and V102 constitute the hydrophobic gate of the CRAC channel (McNally, Somasundaram, Yamashita, \& Prakriya, 2012; Yamashita et al., 2017) (Figure 1). Pore mutations in TM1, such as V102C, cause constitutive channel opening by physically removing gating barriers within the pore (McNally et al., 2012; Yamashita et al., 2017). However, several gain-offunction mutations are known that are not located in the pore, but in the second (TM2-TM3) or third ring (TM4) of interacting transmembrane helices. These mutant variants are interesting because they can potentially trigger gating-related conformational changes that are downstream to STIM1-based activation (Krizova et al., 2019; Yeung, Yamashita, et al., 2020). Unexpectedly, mutation of residue H134 in hO1, which is located in the TM2 region (second ring of TM helices), constitutively activates hO1 as well as Drosophila melanogaster Orai (dOrai) and retains all hallmark properties of CRAC channel activity, such as $\mathrm{Ca}^{2+}$ selectivity (Frischauf et al., 2017; Hou 


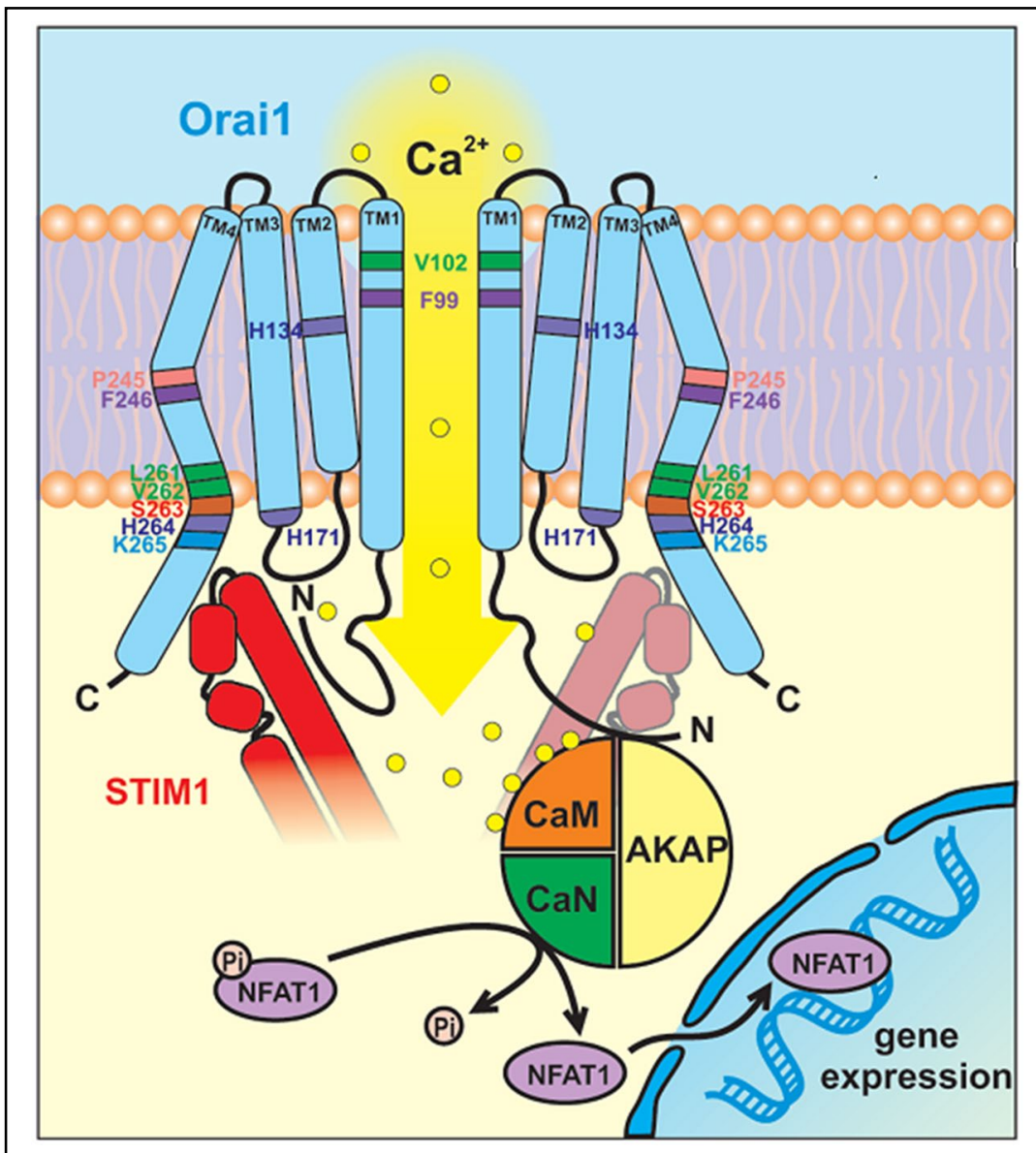

Figure 1. Cartoon representation of human Orai1 showing its four transmembrane (TM) helices TM1-4.

Only two out of six subunits are shown for simplicity, highlighting the hydrophobic gate residues F99 and V102 in the TM1 pore helix, H134 in the TM2, H171 in TM3, P245 and F246 in TM4 and LVHSK residues in the TM4 extension (TM4ext). Binding of STIM1/SOAR (shown in red) to the C-terminus of Orai1 leads to conformational changes within Orai1 propagating from TM4 to TM1 leading to opening of the channel pore and influx of $\mathrm{Ca}^{2+}$. The association of A-kinase anchoring protein (AKAP79) accommodating calmodulin (CaM) and calcineurin $(\mathrm{CaN})$ with the $\mathrm{N}$-terminus of Orai1 delimits the dephosphorylation of NFAT1 at the mouth of the channel, and thus facilitating its rapid translocation to the nucleus, wherein it modifies expression of target genes.

et al., 2018; Yeung et al., 2018). The H134 position in hO1 (H206 in dOrai) seems to constitute a "steric brake", which, when replaced by a residue with a smaller sidechain, causes a significant dilation of the pore (Frischauf et al., 2017; Hou et al., 2018; Hou et al., 2020; Yeung et al., 2018) and "rotation" of the TM1 helix (Bulla et al., 2019; Frischauf et al., 2017; Hou et al., 2018; Yeung et al., 2018). The latter in turn, causes a rearrangement of the porelining hydrophobic gate, formed by residues F99 and V102, which is measured as the rotation of the F99 phenyl ring relative to the axis of the pore (Yeung et al., 2018). Opening of the pore via the rearrangement of F99 seems to be mediated by a so-called sulfur-aromatic latch, where M101 of a given hO1 subunit contacts F99 of the neighboring hO1 subunit, stabilizing the open channel conformation. Conversely, in the closed state, M101 forms contacts with F187 (on TM3), highlighting the role of TM1-TM3 crosstalk in channel gating (Bonhenry, Schober, \& Schindl, 2021; Yeung, Ing, Yamashita, Pomes, \& Prakriya, 2020). Other TM1-TM3 contacts towards the cytoplasmic side have also been shown to be essential for proper channel gating (Dong et al., 2019; Liu et al., 2019; Y. Zhou et al., 2016). On the other hand, the rotation of the TM1 helix was not observed in the molecular dynamics (MD) simulations of the hO1-H134A homology model (Frischauf et al., 2017). Further, the TM1 helix rotation was also not apparent from a recent 3.3 $\AA$ resolution cryo-EM structure of dOrai-H206A (corresponding to 
Augustynek et al.

Gating mechanisms of Orai calcium channels

$\mathrm{H} 134$ in hOrai1). However, the displacement of F171 (corresponding to hO1 F99) away from the pore was observed, which likely resulted from the rigid-body outward movement of each dOrai-H206A subunit (Hou et al., 2020). Overall, a common consensus among these findings suggests that sustained Orai 1 channel opening requires the displacement of F99 away from the pore.

More enigmatic is the mechanism of how mutations in the third ring (TM4) lead to constitutive channel opening. The notorious P245L mutation of h01 was found as a gain-of-function mutation in patients with tubular aggregate myopathy (TAM) (Liu et al., 2019; Nesin et al., 2014), rendering the hO1 channel constitutively active, albeit with a significant loss of selectivity towards $\mathrm{Ca}^{2+}$ (Liu et al., 2019; Palty, Stanley, \& Isacoff, 2015). Structure of P288L dOrai (corresponding to P245L hO1) shows a clear straightening of the kink in TM4 at P288 (Liu et al., 2019). Nevertheless, the relevance of this conformational change has been contested due to establishment of extensive artificial contacts between Orai hexamers, present only in protein crystals but not in physiological conditions, and to the fact that it shows an apparently closed pore geometry (Hou et al., 2020). Interestingly, a non-natural mutant variant of TM4 in hO1, in which the "LVSHK" hinge sequence is replaced by "ANSGA", reproduces all hallmark properties of CRAC current (ICRAC), including inward rectification and $\mathrm{Ca}^{2+}$-selectivity. This contrasts with the P245L variant (Y. Zhou et al., 2016). Thus, the ANSGA substitution, being closest to the STIM1-binding site in TM4ext (Frischauf et al., 2009; Z. Li et al., 2011; Muik et al., 2008; Navarro-Borelly et al., 2008; Palty et al., 2015; Park et al., 2009; Tirado-Lee, Yamashita, \& Prakriya, 2015; Y. Zhou et al., 2016), makes this variant a potentially useful tool to study STIM1-like activation of Orai channels. However, currently no structural information is available about the ANSGA variant and its gating mechanism.

One of the interesting features of the X-ray-based structures of dOrai is that the TM4ext helices protrude either in a "latched" ("quiescent") (Hou et al., 2012) or an "unlatched" state, where the latter state displays the dissociation of the TM4ext bundles, a clear straightening of the kinks in TM4 at both the P288 and the nexus position, and rupture of contacts between TM3 and TM4 (Hou et al., 2018; Liu et al., 2019; Y. Zhou et al., 2019). Importantly, it was concluded that the latched state prevents pore opening, and unlatching is a necessary, but not sufficient condition of channel opening (Hou et al., 2018). However, the physiological relevance of these TM4ext bundles has recently been disputed, as they might be artificially stabilized by crystal contacts, and therefore the role of unlatching in channel activation and pore opening became less clear (Y. Zhou et al., 2019). Further, the interactions between plasma membrane targeted C-terminal TM4ext peptides of hO1 or hO3 could not be observed in a recent FRET-based study (Baraniak et al., 2021) which further questions the formation of antiparallel 
Augustynek et al.

Gating mechanisms of Orai calcium channels

TM4ext interaction pairs between neighboring Orai subunits as reported in the closed dOrai structure (Hou et al., 2012). Future structural studies addressing the closed state of the Orai channel should settle this debate. In context of the channel activation pathway, in particular, TM3-TM4 contacts were proposed to be integral parts (Liu et al., 2019; Y. Zhou et al., 2016; Y. Zhou et al., 2019), since cross-linking studies of positions L174-L261 showed that TM3 and TM4 can also form contacts in the STIM1-activated state, leading to further enhancement of the CRAC current (Y. Zhou et al., 2016). Consistently, disruption of a cluster of hydrophobic residues between TM3 and TM4 (L174, F178 in TM3 and L261, F257 in TM4) attenuates channel activation (Liu et al., 2019; Y. Zhou et al., 2016). Indeed, cryo-EM studies, which avoid the formation of crystal contacts, have reported an apparently semiunlatched state of dOrai P288L (Dong et al., 2019; Liu et al., 2019; Y. Zhou et al., 2019), and also an open state of H206A mutant with intact TM3-TM4 contacts (Hou et al., 2020). It is important to mention that while the relevance of TM4ext latching and unlatching is still debated, dissociation of TM4ext bundles is expected since known interaction sites of Orai1 with STIM1 (L273 and L276) lie in the coiled-coil TM4ext region of Orai (Frischauf et al., 2009; Z. Li et al., 2011; Muik et al., 2008; Navarro-Borelly et al., 2008; Palty et al., 2015; Tirado-Lee et al., 2015). Nevertheless, it is intriguing to speculate whether and how the ANSGA substitution may interfere with the TM4ext regions or TM3-TM4 contacts. The contacts between cytoplasmic regions of TM1 and TM3 may also be essential for the constitutive activity of hO1-ANSGA channel (Y. Zhou et al., 2016). In fact, the K85E and L174D hO1 mutations that abrogate ICRAC (Lis, Zierler, Peinelt, Fleig, \& Penner, 2010; McNally, Somasundaram, Jairaman, Yamashita, \& Prakriya, 2013; Y. Zhou et al., 2016) likely by disrupting the TM1-TM3 and TM3-TM4 cytoplasmic contacts, respectively (Dong et al., 2019; Liu et al., 2019; Y. Zhou et al., 2016), not only abolished the constitutive activity of ANSGA but also that of the P245L mutant channel (Derler et al., 2018; Y. Zhou et al., 2016). It remains puzzling why hO1-ANSGA shows CRAC-like activation while P245L does not, despite their proximity and their location on TM4, indicating that factors other than unlatching or TM4 rearrangement are also affecting the selectivity of ion permeation through the Orai hexamer.

Although all three hOrai paralogs undergo STIM1-mediated activation of CRAC currents, hO2 and hO3 generate 2-3 fold lower currents compared to hO1 (Frischauf et al., 2009; Lis et al., 2007; Mercer et al., 2006). Certain regions of the Orai channels are responsible for paralog-specific differences in gating by STIM1 (Fahrner et al., 2018). Deletion of more than 74 residues from the $\mathrm{N}$-terminus of hO1 completely abolishes STIM1-gated CRAC currents and results in significantly attenuated association of hO1 with STIM1 (Derler et al., 2013; Z. Li et al., 2007; Lis et al., 2010; McNally et al., 2013; Muik et al., 2008; Park et al., 2009; Zheng et al., 2013). Interestingly, N- 
Augustynek et al.

Gating mechanisms of Orai calcium channels

terminal deletion mutants of hO1 can be rescued by replacing loop2 of hO1 (the cytoplasmic region between TM2 and TM3) with that of hO3 (Fahrner et al., 2018). This is surprising given the fact that loop2 is very conserved among Orai paralogs, and the effect of loop2 replacement can be narrowed down to the replacement of 5 amino acids from hO1 with the corresponding ones from hO3 (N147H/K161H/E162Q/E166Q/H171Y), reproducing the rescue effect (Fahrner et al., 2018). These results indicate an extensive crosstalk between TM1 and loop2, which, considering the role of TM1-TM3 contacts in the transduction of gating signals, likely shape paralog-specific differences in gating among human Orai channels. Another recent study elucidates the paralog-specific function of hO1 vs. hO3 gating checkpoints in TM3 (Tiffner et al., 2021). Additional paralog-specific differences in gating of human Orais stem from the differential interaction of their C-termini with STIM1 (Alansary, Bogeski, \& Niemeyer, 2015; Baraniak et al., 2021; Frischauf et al., 2009; S. Li et al., 2019; Niu et al., 2020). Despite these differences in gating between Orai paralogs, the effects of mutations causing constitutive activity mimicking a STIM1-bound state on various Orai channels have not been studied in detail.

The main objective of the current study was to generate new insights into the gating mechanisms of Orai channels and to investigate if there are paralog-specific differences in conformational rearrangements that are linked to Orai channel gating. To address this, we used the H134A and ANSGA hO1 mutants that are reported to largely mimic the STIM1-gated state of the Orai1 channel (Frischauf et al., 2017; Yeung et al., 2018; Y. Zhou et al., 2016). We constructed their corresponding hO2 and hO3 variants and assessed their constitutive activity by $\mathrm{Ca}^{2+}$ imaging on Fluorometric Imaging Plate Reader (FLIPR), electrophysiology as well as NFAT1 nuclear translocation assays. Herein, we show that the conformational coupling between TM2 and TM1 is conserved among all three human Orai paralogs. We investigated whether and how, based on their location, the ANSGA and other residues in Orai paralogs adjacent to the ANSGA sequence might interfere with the TM4ext regions or TM3-TM4 contacts, and thus with the channel activation per se. Our data reveal that the TM4-TM3 coupling implicated in the activation of hO1 likely works differently for the paralogs hO2 and hO3. Overall, our data provides novel insights into the gating mechanisms of Orai channels. 
bioRxiv preprint doi: https://doi.org/10.1101/2021.10.26.465861; this version posted October 26, 2021. The copyright holder for this preprint (which was not certified by peer review) is the author/funder. All rights reserved. No reuse allowed without permission.

Augustynek et al.

Gating mechanisms of Orai calcium channels

Results

The human Orai1 TM2 H134A as well as TM3 F187C corresponding mutations in Orai2 and Orai3 renders

The h01 TM2 residue H134 (Figure 2A), which makes the channel constitutively active when mutated to alanine

by displacing the TM1-F99 residue away from the pore axis, is highly conserved across multiple species (mouse,

Xenopus and Drosophila) as well as in the hO2 and hO3 paralogs (Figure 2B). In order to investigate whether the

$\mathrm{H} 134 \mathrm{~A}$ corresponding mutation in hO2 and hO3 mimics the constitutive activation effect, we generated hO2-

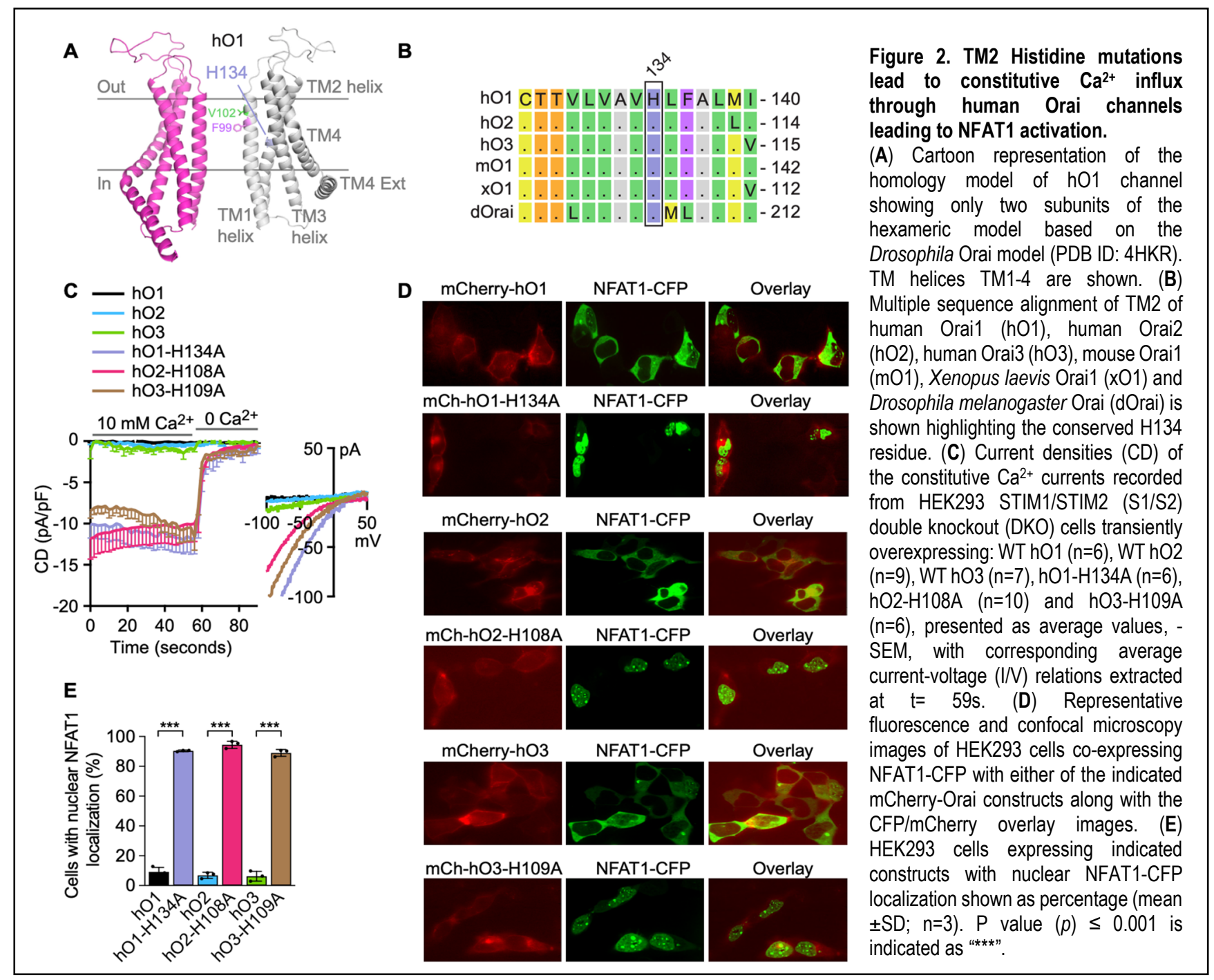


Augustynek et al.

Gating mechanisms of Orai calcium channels

successful ablation of $\mathrm{S} 1$ and $\mathrm{S} 2$ proteins was confirmed at mRNA and protein levels (Figure 2-figure

supplement 1A,B). As expected, SOCE was nearly completely absent in the DKO cells (Figure 2-figure

supplement 1C,D). When expressed in these cells, the hO2-H108A and hO3-H109A mutants showed constitutive

currents similar to hO1-H134A, which disappeared upon removal of $\mathrm{Ca}^{2+}$ from the bath solution, whereas the wild

type (WT) Orai channels did not show any constitutive activity (Figure 2C). Consistent with this finding, nearly

90\% of HEK293 cells expressing either hO1-H134A, hO2-H108A or hO3-H109A mutant displayed translocation

of the CFP labeled NFAT1 transcription factor from cytosol to nuclei, whereas only less than $10 \%$ of the cells

expressing WT Orai proteins showed nuclear translocation of NFAT1 (Figure 2D,E). Overall, these results suggest

that the substitution of $\mathrm{H} 108$ of hO2 and $\mathrm{H} 109$ of hO3 by alanine exerts a similar constitutive activation effect as

known for the h01-H134A mutant.

Recently, it has been proposed that intersubunit F99-M101 interactions support the h01 channel opening, whereas intersubunit M101-F187 (TM1-TM3) interactions facilitate the closing of the channel (Bonhenry et al., 2021; Yeung,

Ing, et al., 2020). Our investigations of these interactions using MD simulations revealed that H134S/H134A hO1

open channel mutants have a higher frequency of intersubunit F99-M101 contacts than WT, whereas no difference

was observed in M101-F187 interactions (Figure 2-figure supplement 2C,D,E). These observations are in line

with the recently published findings on dOrai-H206Q/C channels (Yeung, Ing, et al., 2020). In view of the role of

M101-F187 interactions in maintaining channel closure (Yeung, Ing, et al., 2020); using the FLIPR-based $\mathrm{Ca}^{2+}$ imaging technique, we first validated the earlier published finding that $\mathrm{F} 187 \mathrm{C}$ substitution in hO1 results in its

M101 and F187 residues in hO2 and hO3 (Figure 2-figure supplement 2A,B), we investigated if the mutations

corresponding to hO1 F187C , F161C and F162C in hO2 and hO3, respectively, also lead to their constitutive

activation. Our results showing the constitutive activation of hO2-F161C and hO3-F162C (Figure 2-figure

supplement $2 \mathrm{H}, \mathrm{I})$ suggest that intersubunit TM1-TM3 (M101-F187) interactions facilitating the closed state of the

hO1 channel (Bonhenry et al., 2021; Yeung, Ing, et al., 2020) may also be conserved in hO2 and hO3. 
Augustynek et al.

Gating mechanisms of Orai calcium channels

191

192

193

194

195

196

197

198

199

200

201

202

203

204

205

206

207

208

209

210

211

extension, are mutated to ANSGA residues (Figure 3A). The LVSHK nexus motif is completely conserved in hO1, mouse Orai1 (mO1), Xenopus laevis Orai1 (xO1) and dOrai, whereas the serine at 263 residue position in corresponding hO2 and hO3 sequences is substituted with arginine and alanine, respectively (Figure 3B). The hO1-ANSGA nexus mutant expressed in S1/S2 DKO cells, as expected, exhibited constitutive influx of $\mathrm{Ca}^{2+}$ in both $\mathrm{Ca}^{2+}$ imaging (Figure 3C,D) and patch-clamp measurements (Figure 3G). Strikingly, analogous experiments with the corresponding hO2-ANSGA or hO3-ANSGA variants revealed no constitutive entry of $\mathrm{Ca}^{2+}$ through these mutants (Figure 3E,F,G). Consistently, the hO2-ANGSA or hO3-ANSGA expression did not result in any significant increase in the percentage of HEK293 cells with nuclear translocation of NFAT1 compared to the expression of their WT counterparts (Figure 3J,K). However, nearly 90\% of cells expressing hO1-ANSGA showed nuclear translocation of NFAT1 compared to less than 10\% for WT hO1 (Figure 3J,K). Next, we investigated whether introduction of the ANSGA mutation in the nexus region of hO1 orthologues found in lower organisms can render them constitutively active. Despite the conserved LVSHK motif in both $x O 1$ and dOrai, only the xO1-ANSGA mutant exhibited constitutive $\mathrm{Ca}^{2+}$ entry (Figure $3 \mathbf{H}$ ) while dOrai remained inactive similarly to its WT counterpart (Figure 3I). In order to rule out the possibility that introduction of the ANSGA mutation compromised surface expression of hO2-ANSGA, hO3-ANSGA and dOrai-ANSGA, we introduced an additional, well-established mutation (corresponding to V102A pore-opening mutation of hO1) directly into the TM1 of these mutants. Indeed, the introduction of V76A, V77A and V174A in hO2-ANSGA, hO3-ANSGA and dOrai-ANSGA, respectively, resulted in constitutive $\mathrm{Ca}^{2+}$ influx through these mutants, suggesting that ANSGA mutation does not affect their cell surface localization or proper assembly (Figure 3-figure supplement 1A,B,C). Altogether, these data establish that mutation of nexus residues to ANSGA does not lead to constitutive activation of hO2, hO3 and dOrai, but constitutively activates hO1 and xO1. 
bioRxiv preprint doi: https://doi.org/10.1101/2021.10.26.465861; this version posted October 26, 2021. The copyright holder for this preprint (which was not certified by peer review) is the author/funder. All rights reserved. No reuse allowed without permission.

Augustynek et al. Gating mechanisms of Orai calcium channels

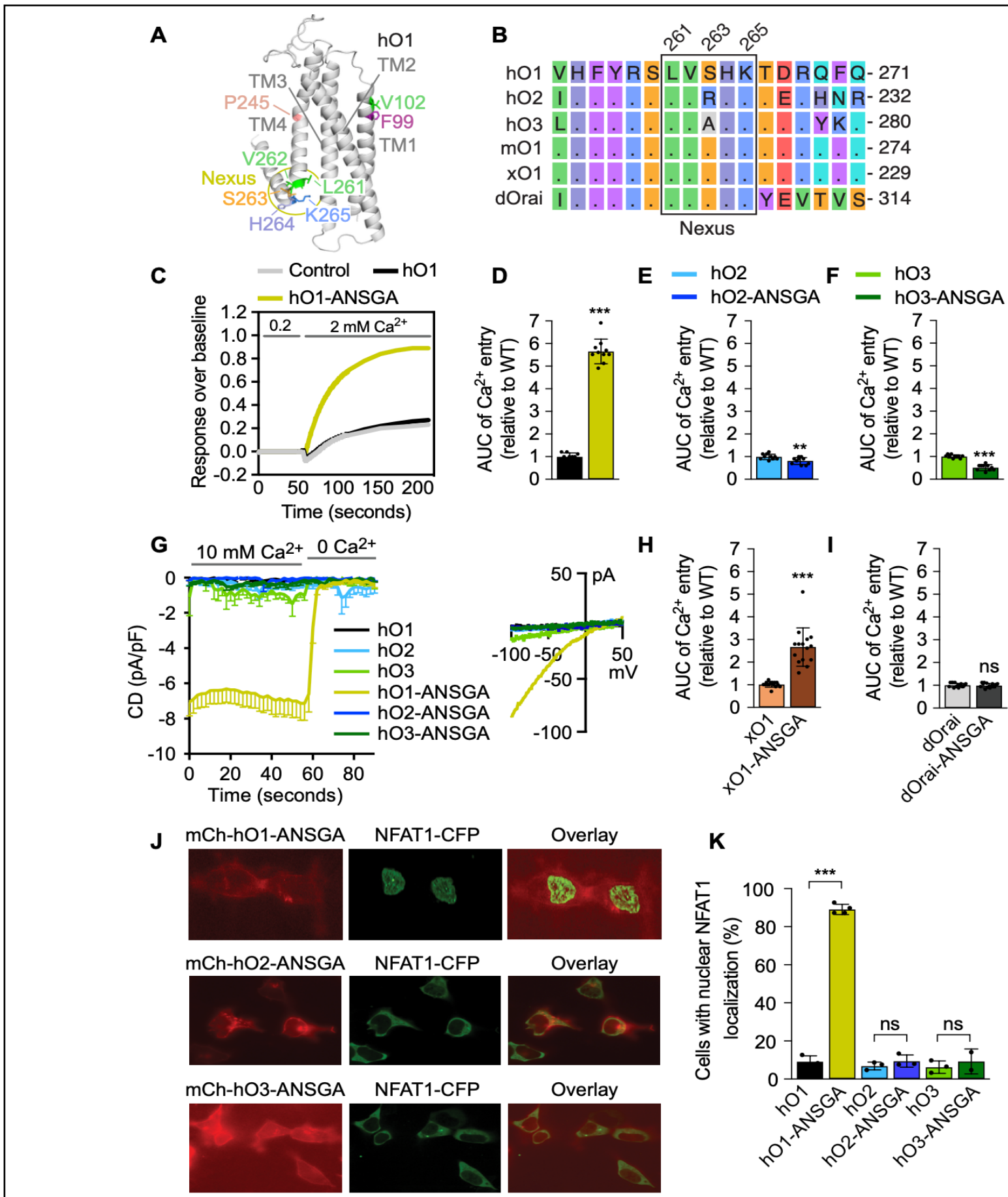

Figure 3. ANSGA substitution of the Orai nexus residues does not exert a constitutive activation effect among Orai homologs.

(A) Cartoon representation of a single subunit of h01 homology model depicting indicated residues along with the LVSHK (261-265) nexus residues. (B) Multiple sequence alignment of TM3 of h01, hO2, hO3, m01, xO1 and dOrai is shown, highlighting the conserved LVXHK residues. (C) Representative constitutive $\mathrm{Ca}^{2+}$ entry traces recorded from HEK293 S1/S2 DKO cells transfected with mCherry control vector, WT h01 and hO1ANSGA constructs with initial baseline recording in $0.2 \mathrm{mM} \mathrm{CaCl}_{2}$, followed by addition of $2 \mathrm{mM} \mathrm{CaCl}_{2}$. The quantified AUC of Ca ${ }^{2+}$ entry peak from HEK293 S1/S2 DKO cells expressing (D) WT hO1 and h01-ANSGA, (E) WT hO2 and hO2-ANSGA and (F) WT hO3 and hO3-ANSGA (mean \pm SD; $\mathrm{n}=10) . p \leq 0.001$ is indicated as "***" and $0.001<p \leq 0.01$ is indicated as "**". (G) Current densities (CD) of the constitutive Ca ${ }^{2+}$ currents recorded from HEK293 S1/S2 DKO cells transiently overexpressing: WT h01 ( $n=6)$, WT hO2 ( $n=9)$, WT hO3 (n=7), h01-ANSGA ( $n=23)$, hO2-ANSGA ( $n=6)$ and hO3-ANSGA $(n=7)$, presented as average values, -SEM, with corresponding average current-voltage $(I / V)$ relations extracted at $t=59 s$. (H) AUC of the constitutive $\mathrm{Ca}^{2+}$ entry traces from WT x01 and x01-ANSGA expressing HEK293 cells (mean $\pm \mathrm{SD} ; n=15$ ). $p \leq 0.001$ is indicated as "***". (I) AUC of the constitutive $\mathrm{Ca}^{2+}$ entry traces from S1/S2 DKO HEK293 cells expressing WT dOrai and dOrai-ANSGA constructs (mean \pm SD; $n=15$ ). $p$ $\geq 0.05$ is indicated as "ns". (J) Representative confocal microscopy images of HEK293 cells co-expressing NFAT1-CFP with either of the indicated mCherry-Orai-ANSGA constructs along with the CFP/mCherry overlay images. (K) HEK293 cells expressing indicated constructs with nuclear NFAT1CFP localization shown as percentaqe (mean $\pm \mathrm{SD} ; \mathrm{n} \geq 3$ ). $p \leq 0.001$ is indicated as "***" and $p \geq 0.05$ is indicated as "ns". 
bioRxiv preprint doi: https://doi.org/10.1101/2021.10.26.465861; this version posted October 26, 2021. The copyright holder for this preprint (which was not certified by peer review) is the author/funder. All rights reserved. No reuse allowed without permission.

Augustynek et al.

Gating mechanisms of Orai calcium channels

The H171Y/F, but not H171A substitution in the TM3 helix of human Orai1-ANSGA abolishes its constitutive activity

We next targeted the molecular determinants that lead to these paralog-specific differences in ANSGA substitution-mediated constitutive activity of Orai channels. For this purpose, we looked in our homology-based model of h01 for the conservation of residues that are in close proximity to the nexus. We identified a histidine residue at position 171 of hO1 that directly faces the nexus region and is conserved in $\mathrm{mO} 1$ and $\mathrm{xO} 1$, but is substituted by a tyrosine residue in hO2, hO3 and dOrai (Figure 4A,B). Based on this and in light of the previous

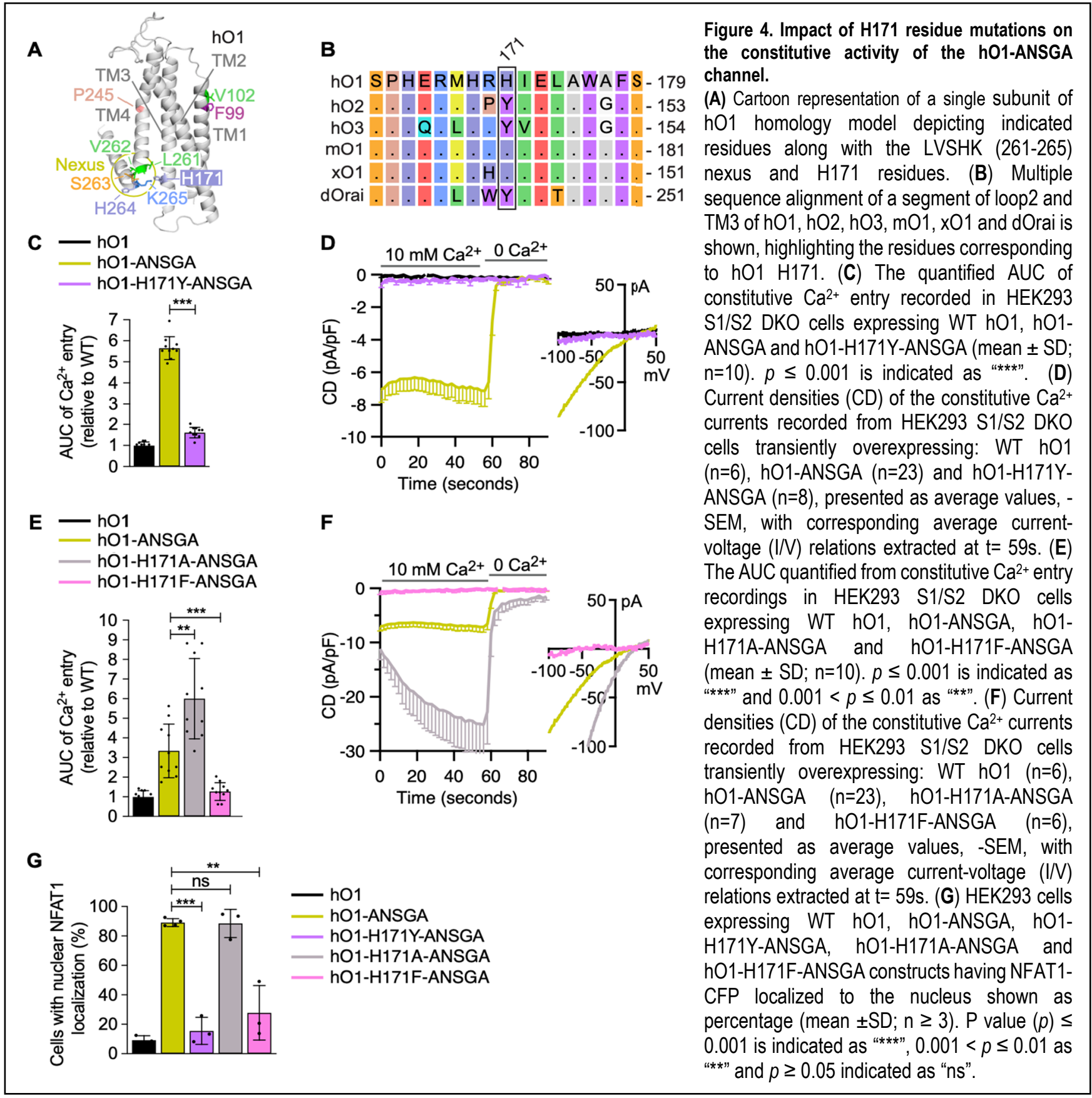


Augustynek et al.

Gating mechanisms of Orai calcium channels

finding that ANSGA substitution of nexus constitutively activates hO1 and $\mathrm{xO}$ (Figure 3D,H), but not hO2, hO3 and dOrai (Figure 3E,F,I), we have hypothesized that the H171Y mutation could interfere with the constitutive activity of the h01-ANSGA mutant. Indeed, $\mathrm{H} 171 \mathrm{Y}$ substitution resulted in complete attenuation of the constitutive activity of hO1-ANSGA channel (Figure 4C,D). To investigate whether mislocalization or altered assembly were the reasons for abolished constitutive activity of the hO1-H171Y-ANSGA channel, we used surface biotinylation experiments and confirmed the expression of hO1-H171Y-ANSGA in the plasma membrane (Figure 4-figure supplement $1 \mathrm{~A}$ ). In addition, the introduction of the $\mathrm{V} 102 \mathrm{C}$ pore mutation rescued the constitutive activity of the hO1-H171Y-ANSGA channel and ensured the correct assembly of the channel (Figure 4-figure supplement 1B). We next tested further substitutions of $\mathrm{H} 171$, of which the H171F, but not $\mathrm{H} 171 \mathrm{~A}$ abolished the constitutive activity of the hO1-ANSGA mutant (Figure 4E,F). Consistently, both the H171Y and the H171F but not the H171A substitutions prevented the nuclear translocation of NFAT1 (Figure 4G, Figure 4-figure supplement 1C).

\section{The H171Y substitution in human Orai1-ANSGA affects the $\alpha$-helical propensity of the TM4 helix extension} as well as the local coupling between TM3 and TM4

We sought to understand the mechanism behind $\mathrm{H} 171 \mathrm{Y}$-mediated inhibition of the constitutive activity of the hO1ANSGA channel. We hypothesized that the H171Y substitution may disrupt the ANSGA-mediated proposed flexion of the nexus hinge (Y. Zhou et al., 2016) and/or TM4-TM3 coupling (Liu et al., 2019; Y. Zhou et al., 2016; Y. Zhou et al., 2019) within hO1-ANSGA, both of which are considered critical for the constitutive activity of hO1-ANSGA. No difference in the helicity of the TM4 helix region (residues 240-250), encompassing the proline bend caused by P245 was observed in the molecular dynamics (MD) simulations of h01-ANSGA and hO1 WT (Figure 5A, Figure 5-figure supplement 1A). Contrariwise, the tubular aggregate myopathy (TAM)-associated P245L mutant showed a significantly higher $\alpha$-helical propensity of the TM4 fragment upstream of L245 (residues 241244) compared to WT (Figure 5-figure supplement 1A). On the other hand, we observed a modest increase in the $\alpha$-helical propensity of the nexus hinge upon the substitution of the nexus residues to ANSGA (Figure 5B). Further, our MD simulations reveal that $\mathrm{H} 171 \mathrm{Y}$ but not $\mathrm{H} 171 \mathrm{~A}$ substitution led to a decrease in $\alpha$-helical propensity of residues 265 to 267 of hO1-ANSGA channel (Figure 5B), which may contribute to the loss of constitutive activity of the hO1-H171Y mutant ANSGA channel.

Next, we addressed whether ANSGA and further H171Y/A substitutions within hO1-ANSGA affect the frequency of contacts between known, functionally important, interacting residues at the interface of TM3 and TM4, namely, 
bioRxiv preprint doi: https://doi.org/10.1101/2021.10.26.465861; this version posted October 26, 2021. The copyright holder for this preprint (which was not certified by peer review) is the author/funder. All rights reserved. No reuse allowed without permission.

Augustynek et al.

Gating mechanisms of Orai calcium channels

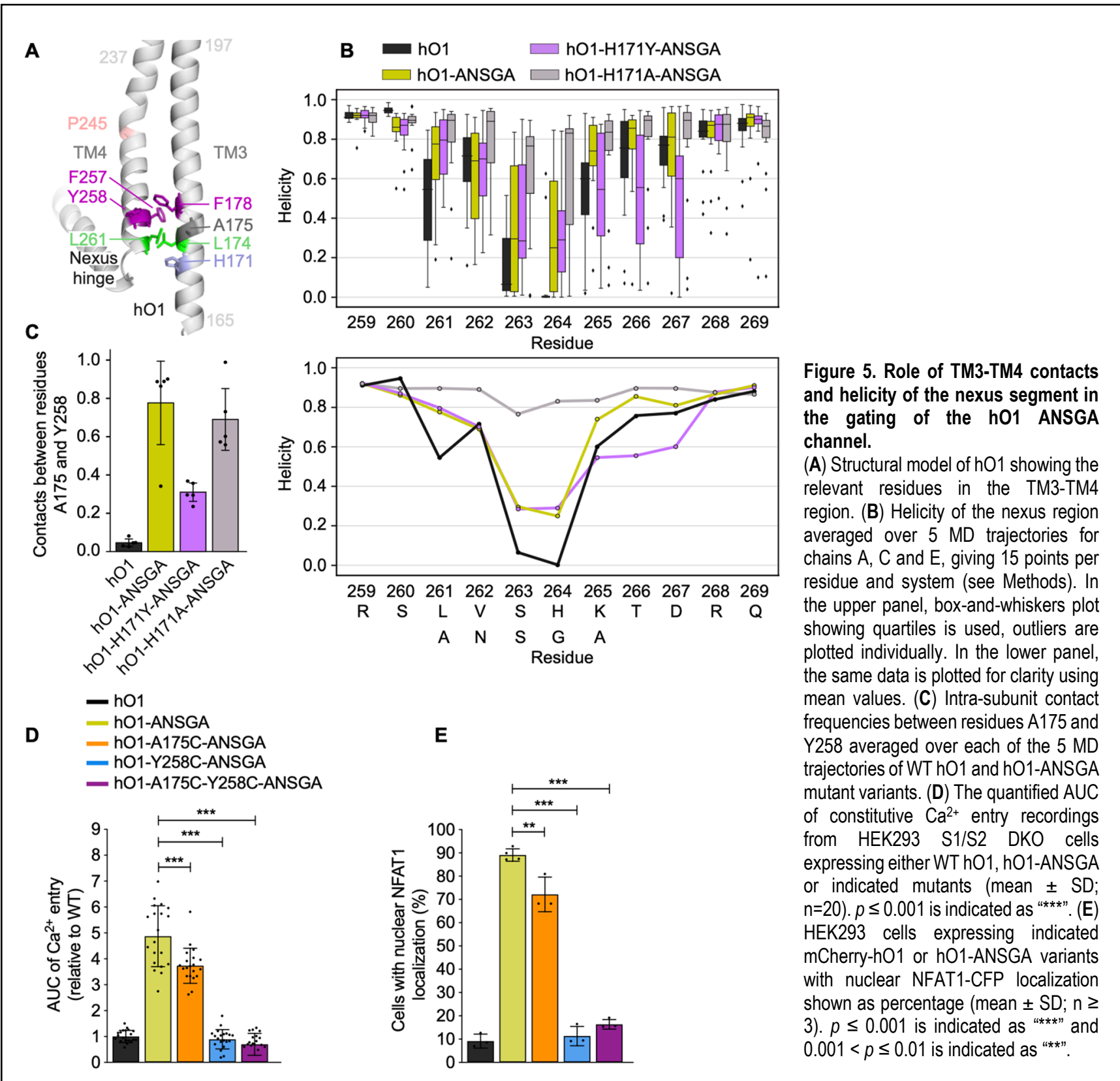

L174(TM3)-L/A261(TM4) and F178(TM3)-F257(TM4) (Figure 5A). No differences could be observed in the contact frequencies between these residues in the MD simulations of h01 WT, hO1-ANSGA, hO1-H171Y-ANSGA and hO1-H171A-ANSGA (Figure 5-figure supplement 1B). Interestingly, further screening and contact analysis led to the identification of different contact frequencies between A175 (TM3) and Y258 (TM4). The MD simulations of the hO1-ANSGA channel showed higher A175-Y258 contact frequency compared to the WT channel. Substitution of $\mathrm{H} 171$ with tyrosine but not alanine residue resulted in a reduction of A175-Y258 contacts (Figure 5C) indicating that the H171Y substitution attenuates the local coupling between TM3 and TM4 in the hO1-ANSGA channel. In line with the previous findings of others, the L174D mutation in the h01-ANSGA channel abolished its 
Augustynek et al.

Gating mechanisms of Orai calcium channels

constitutive activity (Y. Zhou et al., 2016). Moreover, both F178A and F257A substitutions, reported to affect the STIM1-mediated gating of the WT Orai1 channel (Liu et al., 2019), diminished the constitutive activity of the hO1ANSGA channel, further highlighting the importance of these residues in the channel activation (Figure 5-figure supplement 1C). Finally, we addressed whether A175 and Y258 are indispensable for the hO1-ANSGA channel activation. While the $\mathrm{A} 175 \mathrm{C}$ substitution led to only a small reduction, $\mathrm{Y} 258 \mathrm{C}$ resulted in complete loss of constitutive activity of the h01-ANSGA channel without affecting the cell surface localization (Figure 5D, Figure 5-figure supplement 1D). As expected, the A175C-Y258C mutant of the hO1-ANSGA channel did not exhibit constitutive activation. The corresponding effect was observed in NFAT1 activation experiments, wherein expression of hO1-A175C-ANSGA led to only small decrease in percentage of cells showing nuclear translocation of NFAT1 compared to hO1-ANSGA. Conversely, the expression of hO1-Y258C-ANSGA and hO1-A175C-Y258CANSGA mutants reduced the number of cells with nuclear NFAT1 to background level, i.e., the cells expressing hO1 WT (Figure 5E, Figure 5-figure supplement 1E).

We furthermore investigated whether the pore helix rotation reported for the dOrai mutant corresponding to hO1 H134S (Yeung et al., 2018) or pore dilation reported for human H134A Orai1 mutant (Frischauf et al., 2017), could also be observed in the h01-ANSGA mutant, and whether the effect of H171Y substitution in hO1-ANSGA channel alters these parameters. As reported earlier, we could observe rotation of the F99 residue indicative of TM1 helix rotation in hO1-H134S MD simulations (Bulla et al., 2019), which was even more evident in h01-H134A mutant channel (Figure 5-figure supplement 2A). However, this effect could neither be observed in constitutively open hO1-P245L TM4 mutant nor in h01-ANSGA mutant channel in course of 200 ns long simulations (Figure 5figure supplement 2A). On the other hand, in comparison to h01 WT, no considerable pore dilation was clearly evident in the MD simulations of any of the tested hO1 mutants including hO1-ANSGA (Figure 5-figure supplement 2B,C). Since neither TM1 helix rotation nor pore dilation was evident in the MD simulations of hO1ANSGA, we did not analyze these parameters for the H171 mutants of the hO1-ANSGA channel. Overall, these results indicate that the substitution of $\mathrm{H} 171$ by tyrosine in human Orai1-ANSGA decreases the $\alpha$-helical propensity of the TM4 helix extension (residues 265-267) as well as attenuates the local coupling between TM3 and TM4 (A175-Y258). Additionally, we identified Y258 as a critical residue for the constitutive activity of the hO1ANSGA mutant channel. 
bioRxiv preprint doi: https://doi.org/10.1101/2021.10.26.465861; this version posted October 26, 2021. The copyright holder for this preprint (which was not certified by peer review) is the author/funder. All rights reserved. No reuse allowed without permission.

Augustynek et al.

Gating mechanisms of Orai calcium channels

The H171Y substitution does not abolish the constitutive activity of human Orai1 TM1 V102C, TM2

H134A and TM4 P245L and F250C mutants

285

286

287

288

289

290

291

292

293

Next, we aimed to determine if $\mathrm{H} 171 \mathrm{Y}$ mutation also abolishes the activity of other known constitutively open Orai1 mutants, or if its effect is specific to the ANSGA-dependent constitutive activity of hO1. The hO1 TM4 mutants, P245L or F250C as well as TM2 mutant $\mathrm{H} 134 \mathrm{~A}$, did not show any deficit in constitutive $\mathrm{Ca}^{2+}$ influx when additional H171Y mutation was introduced in these channels. Also, the TM1 pore mutant hO1-V102C upon introduction of H171Y substitution resulted in only a minor drop of constitutive activity (Figure 6A,B) suggesting the inhibitory effect of $\mathrm{H} 171 \mathrm{Y}$ is specific to hO1-ANSGA. Further, the effect of $\mathrm{H} 171 \mathrm{Y}$ on the constitutive activity of the hO1ANSGA mutant channel was independent of the TM4 cytosolic extension beyond the ANSGA region, as $\mathrm{H} 171 \mathrm{Y}$ mutation still abrogated the constitutive $\mathrm{Ca}^{2+}$ influx through h01-ANSGA channel lacking the residues 266-301 (hO1-ANSGA- $\triangle \mathrm{CT}$ ) (Figure 6-figure supplement 1).

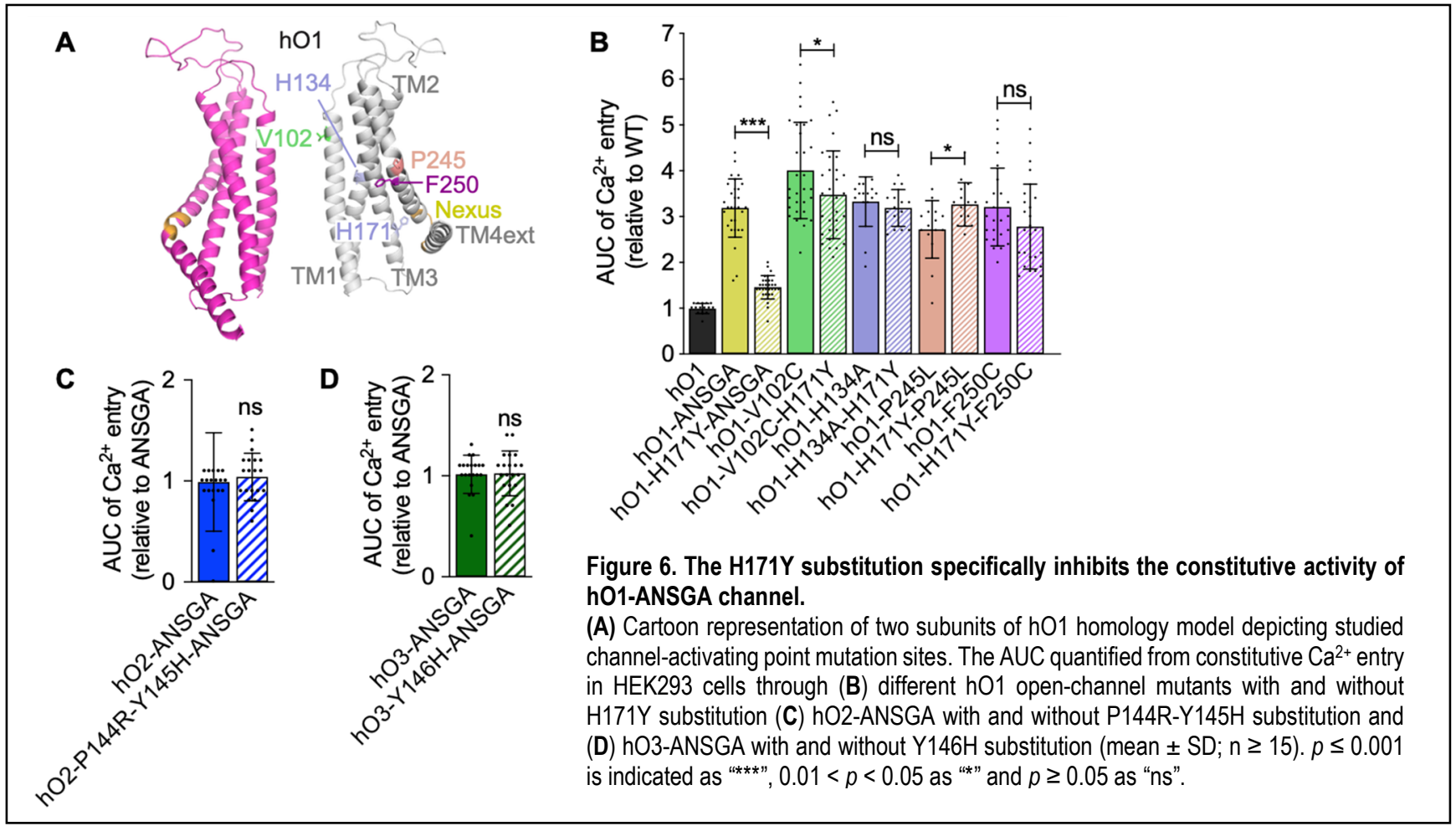

Finally, we addressed whether reverting the native tyrosine in hO2- and hO3-ANSGA channels, corresponding to residue position 171 of hO1, to histidine would render these mutant channels constitutively active. We incorporated the P144R-Y145H and $\mathrm{Y} 146 \mathrm{H}$ substitutions in the hO2- and hO3-ANSGA channels, respectively, to mimic the residues present in hO1. Interestingly, it did not restore their ability to constitutively permeate $\mathrm{Ca}^{2+}$ ions (Figure 
bioRxiv preprint doi: https://doi.org/10.1101/2021.10.26.465861; this version posted October 26, 2021. The copyright holder for this preprint (which was not certified by peer review) is the author/funder. All rights reserved. No reuse allowed without permission.

Augustynek et al.

Gating mechanisms of Orai calcium channels

inactivity of the hO2- and hO3-ANSGA nexus mutants, there may be additional substitutions in these Orai paralogs that do not allow their nexus mutants to be constitutively active.

Substituting TM4 residue(s) of human Orai1 by human Orai2 or Orai3 or mouse Orai1 residue(s) abolishes the constitutive activity of human Orai1-ANSGA

Unlike in case of ANSGA, the hO2 P206L and hO3 P254L mutants corresponding to hO1 P245L showed constitutive $\mathrm{Ca}^{2+}$ entry (Figure 7-figure supplement 1A,B,C). Unexpectedly, however, the hO1 F250Canalogous hO2-F211C and hO3-F259C mutants did not show constitutive activity (Figure 7-figure supplement 1A,B,C), leading us to believe that differences in the TM4 segment between P245 and the nexus residues may contribute to the different outcomes of the ANSGA mutants of human Orai paralogs (Figure 7A). To address this, we first replaced several non-conserved residues spanning 246-255 positions within hO1-ANSGA by the residues present in either hO2 or hO3. The resulting hO1-F246V-I251V-A254T-V255I-ANSGA (mimicking hO2-ANSGA) and hO1-F246V-I249V-I251V-V252A-V255L-ANSGA (mimicking hO3-ANSGA) showed drastically lower or no constitutive $\mathrm{Ca}^{2+}$ influx in comparison to the hO1-ANSGA channel (Figure 7B,C). In the following experiments, we started systematically re-introducing these substitutions into a hO1-ANSGA background to pinpoint the role of individual amino acids in preventing the ANSGA-mediated conformational transition leading to hO1 channel opening. First, we limited the introduced substitutions to F246V and I251V, mimicking both hO2 and hO3, since these are the only two common residues in hO2 and hO3 within the TM4 segment corresponding to residues 246255 of hO1 (Figure 7A). The I251V substitution alone did not affect the constitutive activity of the hO1-ANSGA channel (Figure 7D), whereas the F246V substitution completely prevented its constitutive activation (Figure 7E,F). Consistently, the h01-F246V-ANSGA mutant showed a major drop in percentage of cells showing nuclear NFAT1 translocation compared to the hO1-ANSGA channel (Figure 7G, Figure 7-figure supplement 1D). Importantly, the F246V mutation did not affect the surface expression of the hO1-ANSGA protein (Figure 7-

figure supplement 1G). Interestingly, $\mathrm{mO1}$ contained a cysteine at the residue position 249 corresponding to F246 in hO1 (Figure 7A), and F246C substitution in hO1-ANSGA also abolished its constitutive activity but not the surface expression (Figure 7H, Figure 7-figure supplement 1G). On the other hand, the constitutive activity of the more conservative variant hO1-F246Y-ANSGA was preserved (Figure 7H). Also, a consistent effect of F246C and F246Y substitutions in h01-ANSGA was observed in the NFAT1 nuclear translocation experiments 
bioRxiv preprint doi: https://doi.org/10.1101/2021.10.26.465861; this version posted October 26, 2021. The copyright holder for this preprint (which was not certified by peer review) is the author/funder. All rights reserved. No reuse allowed without permission.

Augustynek et al.

Gating mechanisms of Orai calcium channels

Figure 7. Identification of F246 residue as a gating checkpoint controlling the constitutive activity of the h01-ANSGA channel.

(A) Cartoon representation of a single subunit of h01 model depicting TM4 residues P245-K265 along with the multiple sequence alignment of mentioned residues of $\mathrm{hO} 1, \mathrm{hO}$, h03, m01, xO1 and dOrai is shown. The highlighted residues in boxes are numbered on top according to corresponding $\mathrm{hO} 1$ positions. (B) The quantified AUC of constitutive $\mathrm{Ca}^{2+}$ entry recorded in HEK293 S1/S2 DKO cells expressing WT h01, h01ANSGA and indicated hO2-mimicking and hO3-mimicking TM4 segment h01-ANSGA mutants (mean \pm SD; $\mathrm{n}=20) . p \leq 0.001$ is indicated as "***". (C) Current densities (CD) of the constitutive $\mathrm{Ca}^{2+}$ currents recorded from HEK293 S1/S2 DKO cells transiently overexpressing: WT h01 $(n=6)$, h01-ANSGA (n=23), h01F246V-I251V-A254T-V255I-ANSGA $(n=8)$ and h01-F246V-I249V-I251VV252A-V255L-ANSGA $\quad(n=8)$, presented as average values, -SEM, with corresponding average currentvoltage $(I / V)$ relations extracted at $t=$ 59s. (D) The quantified AUC of constitutive $\mathrm{Ca}^{2+}$ entry recorded in HEK293 S1/S2 DKO cells expressing WT h01, h01-ANSGA and h01I251V-ANSGA mutants (mean \pm SD; $n \geq 20$ ). $p \geq 0.05$ indicated as " $n s$ ". (E) The AUC quantified from constitutive $\mathrm{Ca}^{2+}$ entry in HEK293 S1/S2 DKO cells through h01-ANSGA and h01F246V-ANSGA channel (mean \pm SD; $\mathrm{n} \geq 20$ ). $p \leq 0.001$ is indicated as " "***”. (F) Current densities (CD) of the constitutive $\mathrm{Ca}^{2+}$ currents recorded from HEK293 S1/S2 DKO cells transiently overexpressing: WT h01 $(n=6)$, h01-ANSGA $(n=23)$ and h01F246V-ANSGA $(n=6)$, presented as average values, -SEM, with corresponding average currentvoltage relations extracted at $\mathrm{t}=59 \mathrm{~s}$.

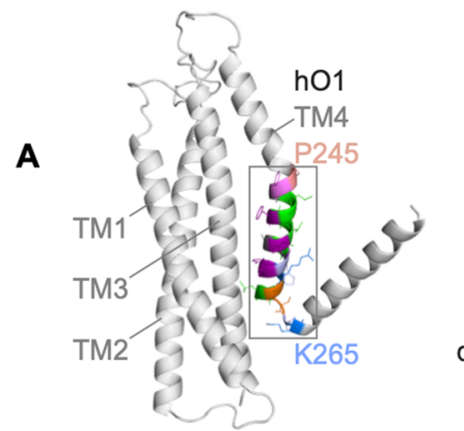

B

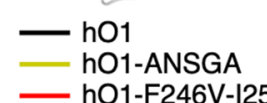

C

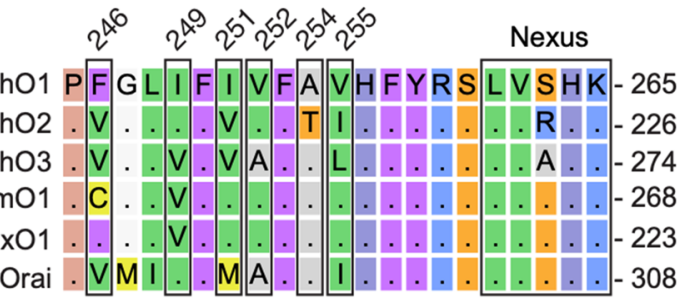

- hO1-F246V-I249V-I251V-V252A-V255L-ANSGA
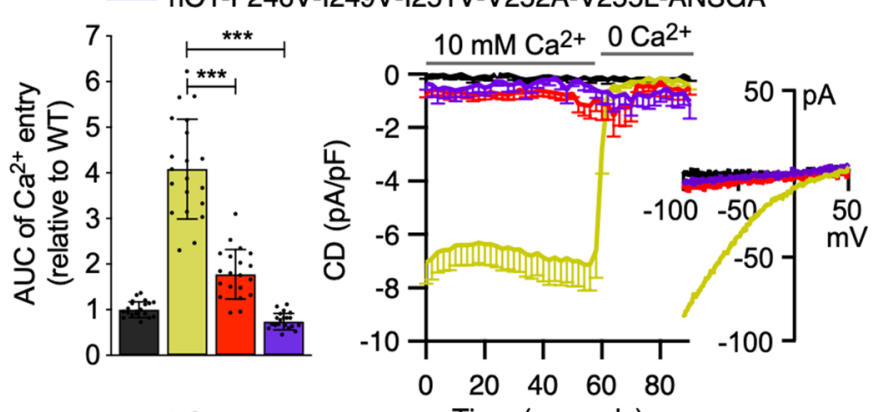

D - h01

- h01-ANSGA

- h01-I251V-ANSGA

E
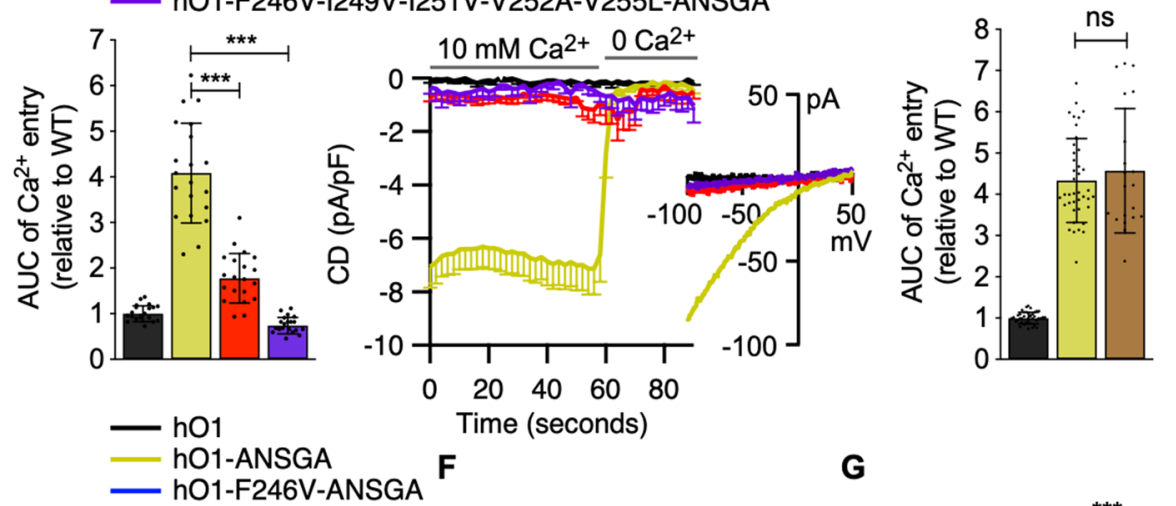

G

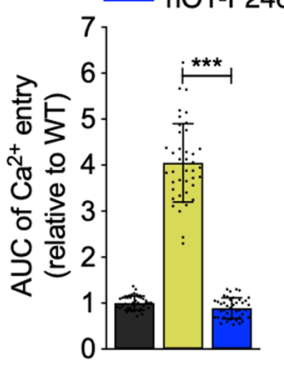

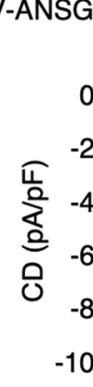

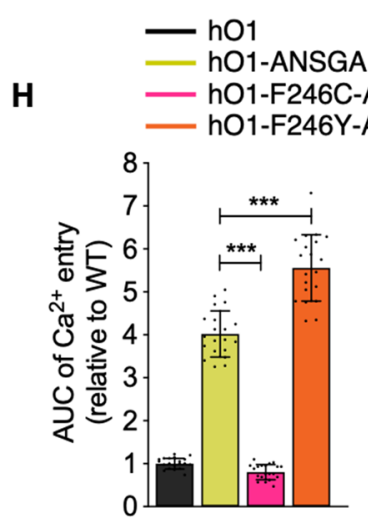

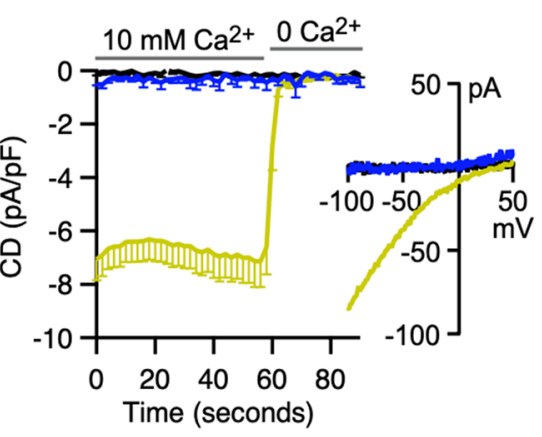

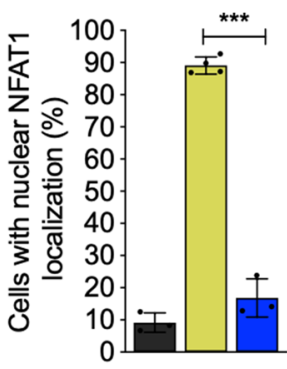

J
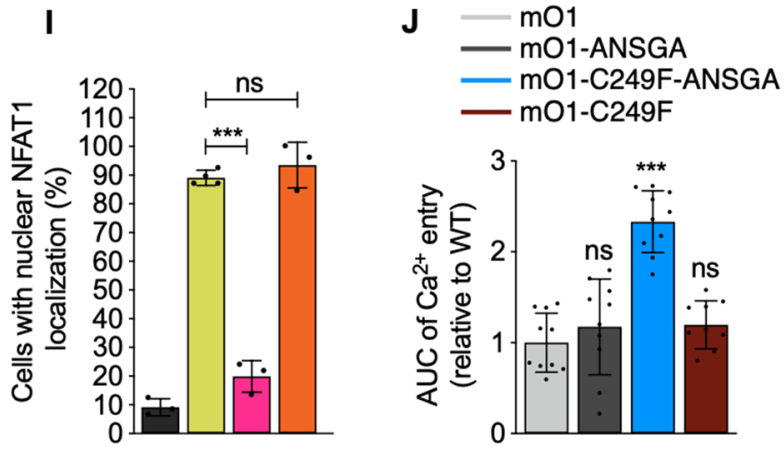

(G) HEK293 cells expressing WT h01, h01-ANSGA and h01-F246V-ANSGA constructs having NFAT1-CFP localized to the nucleus shown as percentage (mean $\pm S D ; n \geq 3$ ). $p \leq 0.001$ is indicated as "***". (H) The quantified AUC of constitutive $\mathrm{Ca}^{2+}$ entry recordings from HEK293 S1/S2 DKO cells expressing h01-ANSGA, h01-F246C-ANSGA and h01-F246Y-ANSGA mutants relative to WT h01 (mean \pm SD; $n=20$ ). $p \leq 0.001$ is indicated as " "***". (I) HEK293 cells expressing WT h01, h01-ANSGA, h01-F246C-ANSGA and h01-F246Y-ANSGA with nuclear localization of NFAT1-CFP are shown as percentage (mean $\pm \mathrm{SD} ; \mathrm{n} \geq 3$ ). $p \leq 0.001$ is indicated as "***" and $p \geq 0.05$ as "ns". (J) The AUC of constitutive Ca ${ }^{2+}$ influx recorded in HEK293 S1/S2 DKO cells expressing WT m01, m01-ANSGA, m01-C249F-ANSGA and m01-C249F mutants (mean \pm SD; $n=10$ ). $p \leq 0.001$ is indicated as "***" and $p \geq 0.05$ as "ns". 
Augustynek et al.

Gating mechanisms of Orai calcium channels

C249 residue at the position of hO1 F246 (Figure 7A). Indeed, mO1-ANSGA was not constitutively active (Figure 7J). However, additional C249F substitution resulted in the activation of the mO1-C249F-ANSGA but not the mO1C249F channel, consistent with our hypothesis (Figure 7J).

We next systematically evaluated the impact of other hO2- or hO3-like substitutions within the hO1 249-255 residues segment on the constitutive activity of h01-ANSGA nexus mutant. The combined A254T and V255I mutagenesis to mimic hO2-like residues in hO1-ANSGA did not abolish the constitutive $\mathrm{Ca}^{2+}$ influx through the resulting h01-A254T-V255I-ANSGA mutant channel (Figure 7-figure supplement 1E,F). On the contrary, the combination of I249V, V252A and V255L mutations to mimic hO3-like residues in hO1-ANSGA abolished the constitutive activity of hO1-I249V-V252A-V255L-ANSGA mutant channel but not its cell surface expression

(Figure 7-figure supplement 1E,F,G). Individual I249V, V252A, V255L or combined I249V-V255L substitutions caused only minor decrease in the constitutive $\mathrm{Ca}^{2+}$ influx through the hO1-ANSGA channel indicating an additive effect of these mutations in abolishment of the constitutive activity of hO1-ANSGA (Figure 7-figure supplement 2A). Consistently, the h01-ANSGA mutants with individual I249V, V252A, V255L or combined I249V-V255L substitutions showed a smaller drop in percentage of cells with nuclear translocation of NFAT1 compared to the h01-I249V-V252A-V255L-ANSGA mutant channel (Figure 7-figure supplement 2B,C). Altogether, these findings highlight the relevance of TM4 residues in the constitutive activation of the hO1-ANSGA channel.

The F246V/C substitution in human Orai1-ANSGA affects the coupling between TM4 and TM3 as well as $\alpha$-helical propensity of the TM4 helix extension

Next, to understand the mechanism of F246V-mediated inhibition of the h01-ANSGA channel, we carried out a set of MD simulations to determine whether the F246 substitutions affect the helicity of the TM4 helix extension spanning residues $259-269$. The F246V mutation in hO1-ANSGA caused a reduction in the $\alpha$-helical propensity of the residues 260-264 which is also evident for the F246C mutation except for residue 262 (Figure 8B). Contrarily, the $\alpha$-helicity of hO1-F246Y-ANSGA showed a similar trend as hO1-ANSGA. Continuing the MD analysis further, we investigated whether the F246 substitutions affect the TM4-TM3 coupling within the hO1ANSGA nexus mutant channel (Figure 8A). While the mutation F246V in the h01-ANSGA channel decreased the contact frequency between A175 and Y258 only slightly, F246C showed considerably lower A175-Y258 contacts. 
bioRxiv preprint doi: https://doi.org/10.1101/2021.10.26.465861; this version posted October 26, 2021. The copyright holder for this preprint (which was not certified by peer review) is the author/funder. All rights reserved. No reuse allowed without permission.

Augustynek et al.

Gating mechanisms of Orai calcium channels

in hO1-ANSGA (Figure 8-figure supplement 1). In summary, our simulations suggest that substitution of F246

in TM4 of the hO1-ANSGA channel to valine or cysteine, but not tyrosine, affects the $\alpha$-helicity of the TM4 helix

extension spanning residues $259-269$ as well as disrupts the local TM3-TM4 coupling.

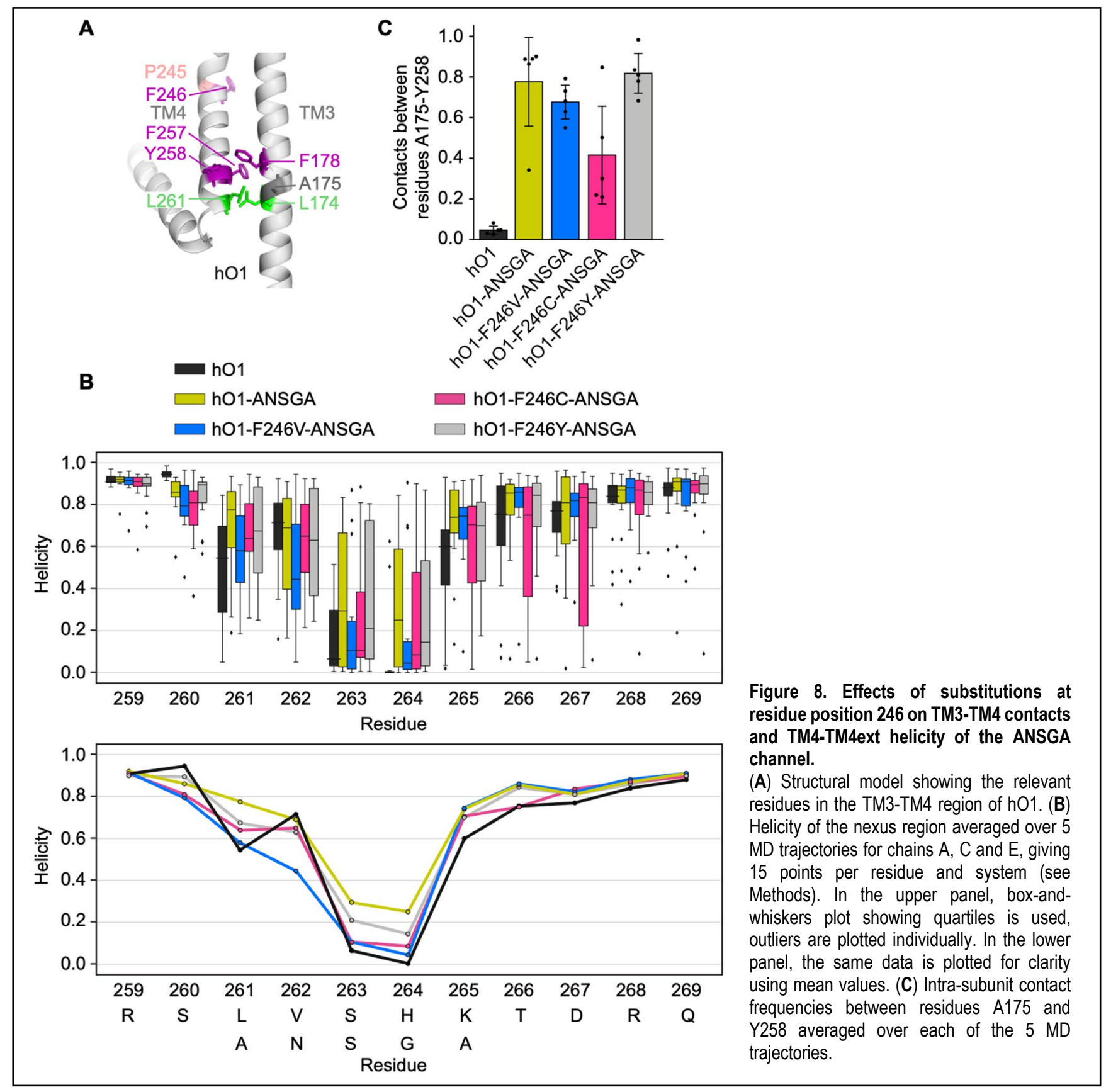
mutants, but abolishes the constitutive activity of human Orai1-F250C mutant 
bioRxiv preprint doi: https://doi.org/10.1101/2021.10.26.465861; this version posted October 26, 2021. The copyright holder for this preprint (which was not certified by peer review) is the author/funder. All rights reserved. No reuse allowed without permission.

Augustynek et al.

Gating mechanisms of Orai calcium channels

363

364

365

366

367

A
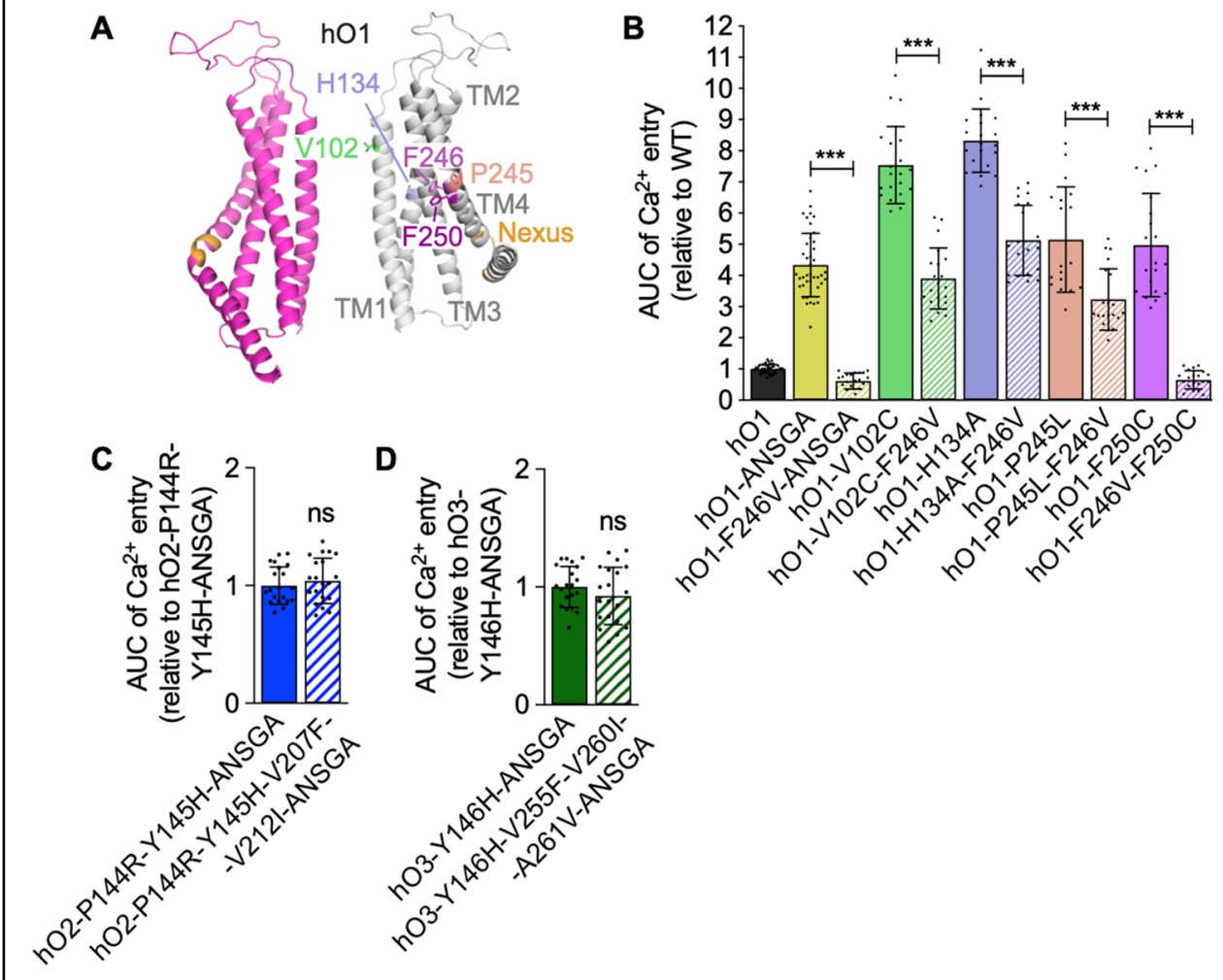

Figure 9. Impact of F246V mutation on the constitutive activity of ANSGA, V102C, H134A, P245L and F250C mutants of h01.

(A) Cartoon representation of two subunits of h01 homology model depicting V102, H134, P245, F246 and F250 residues. Nexus region is also indicated. (B) The AUC quantified from constitutive $\mathrm{Ca}^{2+}$ entry in HEK293 S1/S2 DKO cells through V102C, H134A, P245L, F250C and ANSGA h01 mutants, without and with additional $\mathrm{F} 246 \mathrm{~V}$ substitution (mean $\pm \mathrm{SD} ; \quad \mathrm{n}=20$ ). $\quad \mathrm{AUC}$ quantifications of constitutive $\mathrm{Ca}^{2+}$ measurements in HEK293 S1/S2 DKO cells expressing (C) hO2P144R-Y145H-V207F-V212IANSGA or (D) hO3-Y146H-V255FV260I-A261V-ANSGA mutants (mean $\pm \mathrm{SD}$; $\mathrm{n}=20$ ). $p \leq 0.001$ is indicated as "***" and $p \geq 0.05$ as "ns".

constitutive activity.

As we have identified the F246V (hO2- and hO3-mimicking) and the $1249 \mathrm{~V}-\mathrm{V} 252 \mathrm{~A}-\mathrm{V} 255 \mathrm{~L}$ (hO3-mimicking) substitutions in hO1 paralogs to abrogate constitutive activity of their ANSGA variants, we were interested whether the reverse, hO1-mimicking mutations in a hO2-ANSGA and hO3-ANSGA background would enable constitutive activity of these channels. To this end, we used our hO2-P144R-Y145H-ANSGA and hO3-Y146H-ANSGA mutants as background, which already contained hO1-mimicking substitutions at positions corresponding to $\mathrm{H} 171$ and R170 in hO1 (Figure 4B). In these hO2 and hO3 constructs, we introduced further substitutions mimicking the hO1 F246 (V207F in hO2 and V255F in hO3), 251 (V212I in hO2 and V260l in hO3) and V252 (A261V in hO3) residues (Figure 7A). However, both of these hO1-like hO2-ANSGA and hO3-ANSGA channels remained closed (Figure 9C,D). In addition to the above-mentioned reversal mutations, the hO3-ANSGA mutant further containing the V258I-A261V-L264V substitutions, corresponding to the reversal of h01 I249V-V252A-V255L mutations 
bioRxiv preprint doi: https://doi.org/10.1101/2021.10.26.465861; this version posted October 26, 2021. The copyright holder for this preprint (which was not certified by peer review) is the author/funder. All rights reserved. No reuse allowed without permission.

Augustynek et al.

Gating mechanisms of Orai calcium channels

379 (Figure 7A) that inhibited hO1-ANSGA activity, also remained constitutively inactive (data not shown). Clearly, 380 there are further differences among the human Orai paralogs which render hO2 and hO3 incapable of being 381 activated by the ANSGA nexus mutation.

\section{Human Orai1 H171Y as well as F246V mutants can be gated by STIM1}

Finally, we aimed to determine whether the H171Y and F246V mutations also interfere with the canonical STIM1-

dependent activation of hO1. To this end, we generated relevant hO1 mutants without any mutations in the nexus residues, expressed them in the S1/S2 DKO HEK293 cells along with the STIM1 protein, and measured ICRAC upon stimulation with inositol triphosphate $\left(\mathrm{IP}_{3}\right)$. Similarly, we tested the h01-H171Y-ANSGA and hO1-F246V-

ANSGA mutants. The obtained results showed that both hO1-H171Y and hO1-F246Y mutants could be activated

by STIM1, while the corresponding ANSGA nexus mutants remained inactive (Figure 10). Notably, the hO1H171Y mutant, gated by STIM1, displayed ICRAC characteristics typical for the WT channel (Figure 10A). The hO1-

F246V mutant gated by STIM1, however, evoked ICRAC characterized by different kinetics and current size.

Moreover, it exhibited a clear inactivation component, which was not present in the WT hO1- or hO1-H171Y-

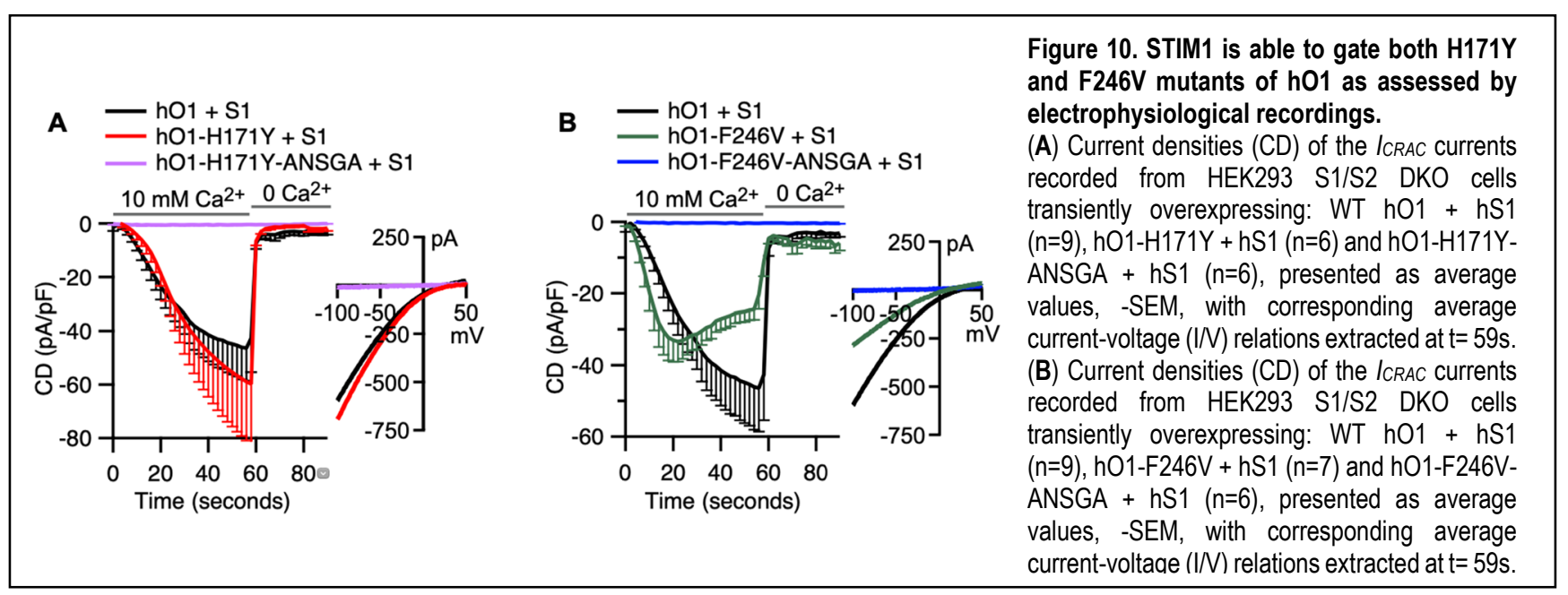


Augustynek et al.

Gating mechanisms of Orai calcium channels

\section{Discussion}

In recent years, several studies have put forward different models for hO1 channel activation, which include the rotation of the TM1 helix (Yamashita et al., 2017), twist-to-open (Dong et al., 2019), counter-ion assisted permeation (Liu et al., 2019) and a pore dilation model (Frischauf et al., 2017; Hou et al., 2018; Hou et al., 2020). However, the exact gating mechanism of the h01 channel by STIM1 is far from being fully understood and is awaiting structural studies of the STIM1-Orai1 complex. Nevertheless, numerous studies have led to the current understanding that the binding of STIM1 at the C-terminus of Orai1 initiates a series of conformational changes that propagate through numerous gating checkpoints within Orai1 subunits and thereby culminate in CRAC channel activation (Fahrner et al., 2018; Frischauf et al., 2017; Palty et al., 2015; Tiffner et al., 2020; Yeung et al., 2018; Y. Zhou et al., 2016). A major goal of our study was to investigate whether these checkpoints and gating elements are conserved among the three human Orai paralogs.

We first investigated whether the TM1-TM2 conformational coupling was also conserved in hO2 and hO3 by 406 mutagenesis of the residues corresponding to $\mathrm{H} 134$, a "checkpoint" residue of this coupling in hO1 (Frischauf et al., 2017; Yeung et al., 2018). The constitutive $\mathrm{Ca}^{2+}$ current observed in both hO2-H108A and hO3-H109A (Figure 2C) suggests a conserved TM1-TM2 interplay in the gating of human Orai channels. Further, constitutive $\mathrm{Ca}^{2+}$ influx through dOrai-H206A was reported recently (Hou et al., 2020). Moreover, in line with our data, a recent report published during the preparation of our manuscript demonstrated constitutive current through the H109A mutant of hO3 but did not test the same for hO2 (Tiffner et al., 2021). Also, the study of Tiffner et al. did not investigate if hO3-H109A or hO2-H108A would lead to nuclear translocation of NFAT1 (Figure 2D,E). On the other hand, the H134A mutant of hO1 has been shown to effectively cause the translocation of NFAT1 to the nucleus 414 (Frischauf et al., 2017). In summary, our results point in the direction that the sequence of events during the gating mechanism downstream of the $\mathrm{H} 134$ checkpoint are conserved in a wide range of Orai proteins.

While both earlier studies related to the $\mathrm{H} 134$ checkpoint suggest a conformational coupling mechanism between

417 TM2 and TM1 of hO1 leading to channel opening, different mechanistic explanations have been proposed. 418 Frischauf et al. suggest that TM2 H134 forms a hydrogen bond with the TM1 S93 residue and this serves as a 419 hydrogen bond-dependent trigger that gates hO1 channels (Frischauf et al., 2017). On the contrary, Yeung et al. 420 argue against the formation of this hydrogen bond and rather suggest that substitution of the bulky side chain of 421 H134 by smaller amino acids allows rotation of the TM1 F99 away from the pore axis (Yeung et al., 2018), a 
Augustynek et al.

Gating mechanisms of Orai calcium channels

phenomenon that was not observed by Frischauf and co-workers (Frischauf et al., 2017). Although the rotation of the TM1 helix was also not evident from the recent structure of dOrai-H206A (corresponding to hO1-H134A), the rigid-body outward movement of each dOrai-H206A subunit resulted in repositioning of F171 (corresponding to h01 F99), which slides away from the pore, widening the hydrophobic region (Hou et al., 2020). In contrast, we could observe the TM1 (F99) rotation in h01-H134A MD simulations as well as reproduce our earlier findings using a homology-based model of hO1 bearing the H134S substitution (Bulla et al., 2019) (Figure 5-figure supplement 2A). Overall, our MD simulations confirm that sustained Orai1 channel opening requires the displacement of F99 away from the pore.

Our results also indicate that the mechanistic function of the sulfur-aromatic latch is conserved in all three human Orai channels. The M101 residue in h01 TM1 has been suggested to function as a "gate latch" by engaging the hydrophobic gate (F99) of the neighboring subunit to sustain the open conformation of the hexameric channel (Yeung, Ing, et al., 2020). Consistently, our MD simulations have confirmed a higher contact frequency between F99-M101 in h01-H134A/S compared to the WT channel (Figure 2-figure supplement 2C,D,E). Our finding is in line with the report on narrowing of the distance between F171 and M173 of dOrai (corresponding to hO1 F99 and M101, respectively) in the open state of the channel (dOrai H206C/Q) compared to WT (Yeung, Ing, et al., 2020). In the other, resting state of the "gate latch", it has been suggested that M101 partners with F187 in the TM3 of the adjacent subunit, stabilizing the M101-F187 resting interaction. This, in turn, may arrest the hO1 channel in a closed state, likely due to the release of F99 side chain into the pore-facing conformation (Yeung, Ing, et al., 2020). Interestingly, hO1-F187C was reported constitutively active (Yeung et al., 2018) and in light of the recent findings from Prakriya's laboratory, it is reasonable to envision that due to the inability of $\mathrm{C} 187$ to engage in the M101-dependent gate latch, M101 instead permanently arrests F99 out of the pore axis, supporting the constitutively active state of the hO1-F187C channel (Yeung, Ing, et al., 2020). Our findings that the hO1-F187C and the corresponding hO2-F161C and hO3-F162C channels are constitutively active (Figure 2-figure supplement $2 \mathrm{~F}, \mathrm{G}, \mathrm{H}, \mathrm{I})$ suggest that the recently proposed M101-F187 interactions which support hO1 channel closing (Bonhenry et al., 2021; Yeung, Ing, et al., 2020) are also conserved in hO2 and hO3. Overall, our data show that the inter-subunit conformational coupling of hO1 between TM1-TM2 (F99-H134), as well as TM1-TM3 (M101-F187), are also conserved in hO2 and hO3 paralogs.

Moving more distal from the pore, we showed that the TM4 mutations corresponding to the position hO1 P245, namely P206C and P254C in hO2 and hO3, respectively, rendered them constitutively active (Figure 7-figure 
Augustynek et al.

Gating mechanisms of Orai calcium channels

supplement $1 \mathrm{~A}, \mathrm{~B}, \mathrm{C})$. Consistently, constitutive current through hO3-P254L has also been recorded in a previous report (Derler et al., 2018). The structure of P288L dOrai (corresponding to P245L hO1) shows a clear straightening of the kink in TM4 at P288 (Liu et al., 2019). The conserved constitutive activation effect of P245, P206 and P254 substitutions in hO1, hO2 and hO3, respectively, suggests that these mutations may trigger similar structural rearrangements among Orai paralogs. In our MD simulations, the tubular aggregate myopathy (TAM)associated P245L mutant showed a significantly higher $\alpha$-helical propensity of the TM4 fragment upstream of L245 (residues 241-244) compared to WT (Figure 5-figure supplement 1A), indicating the unbending of the proline kink as a possible mechanism of how this mutation opens the channel.

In contrast to the above-mentioned gating checkpoints that we show to be conserved among human Orai channels, we also report positions that could represent steric brakes that are not conserved among the three human Orai channels. Interestingly, these positions lie further away from the pore, in the TM4 and TM4ext regions.

One of these positions is residue F250 within TM4 of hO1, the mutation of which (F250C) was identified in a cysteine screen of hO1 TMs (Yeung et al., 2018) to lead to the constitutive activation of hO1 (Tiffner et al., 2020; Yeung et al., 2018). Our data shows that unlike the mutants of hO2 and hO3 corresponding to hO1 P245L, the hO2-F211C and hO3-F259C mutants, corresponding to hO1-F250C, do not exhibit any constitutive activity (Figure 7-figure supplement $1 \mathrm{~A}, \mathrm{~B}, \mathrm{C})$.

Importantly and central to our work, we also show that the ANSGA "nexus" mutations, further along the protein chain in the hinge region between TM4 and TM4ext, likely also exert their activating effect through a non-conserved mechanism. Based on hO1-ANSGA, we expected the ANSGA mutants of hO2 and hO3 to also display constitutive activity and NFAT1 activation. However, this was not the case (Figure 3C,D,E,F,G,J,K). Also, despite dOrai, xO1 and mO1 all having the LVSHK nexus residues conserved, only the ANSGA mutant of $\mathrm{xO} 1$ exhibited the constitutive activity (Figure $3 \mathrm{H}, \mathrm{I}$ and Figure $7 \mathrm{~J}$ ).

Although all of the P245, F250 and the LVXHK nexus residues of hO1 are conserved among different human Orai paralogs as well as in Orai channels of lower organisms, the neighboring residues within TM4 are not very well conserved, e.g. Drosophila Orai1 protein, which shares only $47 \%$ overall identity with hO1, has several additional non-conserved residues downstream from P288 corresponding to P245 of hO1 (Figure 7A). Thus, the differences in sequence in this region might interfere with constitutive activation by ANSGA in certain Orai channels and may also explain differences in gating mechanism in various Orai paralogs and orthologs. 
Augustynek et al.

Gating mechanisms of Orai calcium channels

479 Current knowledge on the mechanism by which STIM1 or ANSGA nexus mutations activate Orai1 is incomplete and does not explain our observed lack of activation by the ANSGA mutation in hO2, hO3 and certain Orai orthologs. The gating-related conformational changes that initiate within the TM4 helix of hO1 upon binding of STIM1 to its C-terminus and propagate to the channel's pore-forming TM1 presumably rely on flexion of the hO1 C-terminal nexus hinge residues (Y. Zhou et al., 2016). In support of this mechanism, we observed a notable increase in the calculated helicity of the LVSHK region when substituted with ANSGA residues, which may be indicative of the flexion of TM4ext (Figure 5B). Disrupting the flexibility of the LVSHK hinge, for example by the S263P mutation, abolished ICRAC (Tirado-Lee et al., 2015) even when h01 was covalently linked with two active fragments of STIM1, suggesting a critical role of hinge flexibility in gating of hO1 by STIM1 (Palty et al., 2015). The motifs corresponding to LVSHK in hO2 and hO3 contain either an arginine or an alanine residue, respectively, in the place of serine (Figure 3B). These substitutions, however, are not expected to change the overall structure or flexibility of the LVSHK hinge, as the mutation of S263 to either arginine or alanine did not affect the interaction between hO1 and channel activation domain of STIM1 (Tirado-Lee et al., 2015). Therefore, hO2 and hO3 are envisioned to have an overall similar structural architecture of the nexus hinge as hO1. Furthermore, mutations of hO2 and hO3 residues corresponding to the earlier mentioned L273 and L276 residues of hO1 also affected the interaction of hO2 and hO3 with STIM1, as well as diminished the ICRAC (Baraniak et al., 2021; Frischauf et al., 2009; Frischauf et al., 2011; Niu et al., 2020). The mechanism how ANSGA causes the constitutive activity of the hO1 channel is also likely different from that of the P245L mutation, since the helicity changes observed in our MD simulations upon ANSGA substitution of LVSHK residues in hO1 remained restricted around the nexus region without affecting the helicity of the TM4 helix region (residues 240-250) encompassing the proline bend caused by P245 (Figure 5A, Figure 5-figure supplement 1A).

Interestingly, we were able to uncover three positions that seem to interfere with ANSGA-induced activation of hO1, all of which point at the importance of TM4-TM3 contacts in transducing gating-relevant conformational changes from the distal TM rings towards the central pore. The first of these positions, which we have identified through multiple sequence alignments and homology-based modeling, is $\mathrm{H} 171$, located in the cytosolic extension of TM3 helix (TM3ext), proximal to the LVSHK motif, and not conserved in hO2, hO3 and dOrai (Figure 4A,B). When $\mathrm{H} 171$ was substituted by tyrosine, which is the native residue at the corresponding position in hO2, hO3 and dOrai, both the constitutive activation of hO1-ANSGA and NFAT1 activation were lost (Figure 4C,D,G). 
Augustynek et al.

Gating mechanisms of Orai calcium channels

508

509

510

511

512

513

514

515

516

517

518

519

520

521

522

523

524

525

526

527

528

529

530

531

532

533

534

535

536

channel mutants such as TM1 V102C (McNally et al., 2012; Yamashita et al., 2017), TM2 H134A (Frischauf et al., 2017; Yeung et al., 2018), TM4 P245L (Nesin et al., 2014; Palty et al., 2015) and TM4 F250C (Yeung et al., 2018) (Figure 6A,B). Similarly, the H171Y substitution in hO1 with native nexus "LVSHK" residues did not affect STIM1mediated gating of the channel (Figure 10A). Nevertheless, the importance of the $\mathrm{H} 171$ residue in gating is also corroborated by a recent report showing that the H171D substitution, identified through randomized mutations, minimized the activity of the engineered light-operated h01-P245T channel in dark, while retaining its activity under blue light illumination (He et al., 2021). Residue $\mathrm{H} 171$ of hO1 appears to be a feature in the Orai1 proteins of vertebrates, whereas a conserved tyrosine residue takes the corresponding position in invertebrates. A study suggesting diversification of Orai gating mechanisms between invertebrates and mammals showed that the F192R-Y193H substitution in the Orai channel of C. elegans to mimic hO1 residues R170-H171 led to STIM1independent constitutive activation of $C$. elegans Orai channel, as well as nuclear translocation of NFAT1 (Kim et al., 2018). Thus, we propose that the $\mathrm{H} 171$ position is essential to Orai channel gating, nevertheless its role in hO1 might be limited to local, "weak" stabilization of TM3-TM4 contacts. Thus, a H171Y substitution, native to hO2 and hO3 at this position, abrogates the effect of the ANSGA mutation in hO1, but not that of other activating mutations, and it also does not affect normal STIM-mediated gating.

In pursuit of understanding the mechanism of action of the H171Y-mediated selective inhibition of hO1-ANSGA constitutive activity, we show that TM3-TM4 contacts are essential for ANSGA-induced activation. In particular, TM3-TM4 interaction pairs F178-F257 (Liu et al., 2019) and L174-L/A261 (Y. Zhou et al., 2016) have been previously shown to be important for hO1 channel activation, and we found that these residues, as well as Y258 (TM4) are indispensable for the activity of the hO1-ANSGA channel as well (Figure 5D,E and Figure 5-figure supplement 1C). While the ANSGA substitution did not affect the contact frequencies of these TM3-TM4 residue pairs in our MD simulations, it did result in significantly enhanced contact frequencies of another TM3-TM4 residue pair, A175 and Y258, which were in turn impaired by a further H171Y substitution (Figure 5A,C). Furthermore, we found that all the residues past the ANSGA sequence do not take part in shaping the constitutive activity of the channel, since the constitutive activity of hO1-ANSGA- $\Delta$ CT channel was not compromised (Figure 6-figure supplement 1 and Figure 9-figure supplement 1), which is consistent with earlier reports (L. Zhou et al., 2018; Y. Zhou et al., 2016). In addition, the H171Y mutation still inhibited the constitutive activity of the hO1-ANSGA channel even after the removal of residues 266-301 (Figure 6-figure supplement 1). Therefore, even though the H171Y substitution reduced the $\alpha$-helical propensity of hO1-ANSGA residues $265-267$ C-terminal to the 
Augustynek et al.

Gating mechanisms of Orai calcium channels

537

538

539

540

541

542

543

544

545

546

547

548

549

550

551

552

553

554

555

556

557

558

559

560

561

562

563

564

ANSGA motif (Figure 5B) in our MD simulations, the last 36 residues beyond residue 265 are dispensable for ANSGA-induced gating. Based on these results, we believe that the impairment in the local coupling between TM3-TM4 (A175-Y258) may be the relevant reason for the disruption of hO1-ANSGA channel activity by the H171Y substitution.

The second position that we found critical in controlling the constitutive activity of hO1-ANSGA to allow influx of $\mathrm{Ca}^{2+}$ in a constitutive manner with subsequent nuclear translocation of NFAT1 was residue F246. F246 in hO1 is conserved in $\mathrm{xO} 1$ but neither in hO2, hO3 and dOrai, where it is replaced by a valine residue, nor in mouse Orai1, where it is replaced by cysteine. Herein, both F246V and F246C substitutions in hO1-ANSGA prevented the constitutive $\mathrm{Ca}^{2+}$ influx and subsequent nuclear translocation of NFAT1 (Figure 7E,F,G,H,I and Figure 7-figure supplement 1D). Thus, we can speculate that the valine residue in hO2 and hO3 at the positions corresponding to F246 of hO1 participates in exerting a brake on the putative constitutive activity of the hO2- and hO3-ANSGA channels.

Although the F246V mutation did not abolish the activity of other constitutively active hO1 mutants such as V102C, $\mathrm{H} 134 \mathrm{~A}$ and P245L, it significantly decreased the measured influx of $\mathrm{Ca}^{2+}$. However, unexpectedly, substitution of F246 to valine completely abolished the constitutive influx of $\mathrm{Ca}^{2+}$ through the hO1-F250C and hO1-ANSGA mutants (Figure 9A,B, Figure 7E). Since F246V did not alter the surface trafficking of hO1-ANSGA (Figure 7figure supplement 1G), it is likely that this mutation does not alter the trafficking of V102C, H134A, P245L or F250C mutants either. Similarly to H171Y, our MD simulations suggest that TM4 mutations (F246V/C) that interfere with the constitutive activation of hO1-ANSGA also impair local coupling between TM3-TM4 (A175-Y258) and $\alpha$-helicity of the nexus segment (Figure 8). Since we also observed a significant decrease in the amplitude of STIM1-activated ICRAC mediated by hO1-F246V in comparison to the WT hO1 (Figure 10B), it is likely that this TM4 residue is important in regulating the channel activity. In comparison to the H171 position, F246 seems to be a "strong" effector of gating in hO1, since it is able to abolish the effects of the activating mutations ANSGA and F250C, while at the same time to significantly attenuate $\mathrm{Ca}^{2+}$ influx mediated either by the other tested constitutively active mutant variants of hO1, or by STIM1-mediated gating.

In addition, as a third position affecting the gating of hO1-ANSGA, we found that a combination of other residue substitutions mimicking the residues of hO3 TM4 on hO1 (I249V-V252A-V255L) leads to abrogation of the constitutive activity of hO1-ANSGA (Figure 7-figure supplement 1E,F and Figure 7-figure supplement 2). 
Augustynek et al.

Gating mechanisms of Orai calcium channels

565 These positions seem to have an additive effect, which implies that there could be several other positions making contributions to shape the energetic barrier of constitutive activity.

567 Interestingly, while the substitution of $\mathrm{H} 171$ by tyrosine abrogated the constitutive activity of hO1-ANSGA, the reverse substitution of the corresponding hO2 Y145 and hO3 Y146 residues by histidine was not sufficient, either alone or in combination with TM4 mutations, to evoke a constitutive $\mathrm{Ca}^{2+}$ influx through hO2- or hO3-ANSGA mutant channels (Figure 6C,D, Figure 9C,D). These data provide further evidence that other residue differences in hO1 and hO2/hO3 can shape the overall energetics of channel gating. Mouse Orai1 protein, which shares $90 \%$ sequence identity with hO1, has a cysteine at the residue position corresponding to F246 of hO1 with most of the downstream residues conserved compared to hO1 (Figure 7A). Strikingly, the ANSGA mutant of mO1, which was otherwise constitutively inactive, resulted in a modest constitutive activation when C249 was substituted with a phenylalanine residue to mimic the native residue (F246) of hO1 (Figure 7J). This interesting result suggests that at least in certain Orai channels, the energetic barrier of activation can be lowered enough by a single point mutation so that activating mutations such as ANSGA can induce constitutive activity in the channel.

Earlier, it was shown that the addition of the ANSGA substitution in the otherwise non-selective h01-V102C pore mutant channel renders selectivity towards $\mathrm{Ca}^{2+}$ and that the mutations that abrogate STIM1-mediated activation of hO1, such as the L174D mutation, also eliminate the constitutive activity of hO1-ANSGA channel (Y. Zhou et al., 2016). Based on these findings it was suggested that the ANSGA substitution of the "LVSHK" nexus residues of hO1 mimics the STIM1 gating trigger and causes a conformational change in the hO1 TM4ext leading to the opening of the channel pore formed by TM1. However, we show here that two different hO1 single point mutations, one in TM3ext (H171Y) and another in TM4 (F246V), adopted from other Orai homologs, completely abolish the constitutive activity of the 01-ANSGA channel but do not shut down the permeation of $\mathrm{Ca}^{2+}$ through STIM1-gated hO1 channels. This provokes us to reconsider how well the ANSGA mutant resembles the actual STIM1-gated open state of hO1. As the conformational trigger by the ANSGA substitution leading to the opening of hO1 channel is obstructed by changes that still allow gating by STIM1, it is likely that either the ANSGA channel does not follow the same order of conformational rearrangements that lead to the channel opening, or that the gating stimulus provided by STIM1 supersedes the stimulus provided by ANSGA (Figure 11). In other words, we could expect the ANSGA nexus mutations to be "weak" activators, while STIM1 binding provides a "strong" stimulus that is able to overcome higher energetic barriers to gate the channel as depicted in our hypothetical model (Figure 11B,C). We propose that mutations of TM3ext H171 and TM4 F246 in hO1 interfere with the ANSGA-mediated gating signal 
bioRxiv preprint doi: https://doi.org/10.1101/2021.10.26.465861; this version posted October 26, 2021. The copyright holder for this preprint (which was not certified by peer review) is the author/funder. All rights reserved. No reuse allowed without permission.

Augustynek et al.

Gating mechanisms of Orai calcium channels

594

\section{B WT Orai1 + STIM1}

A WT Orai1

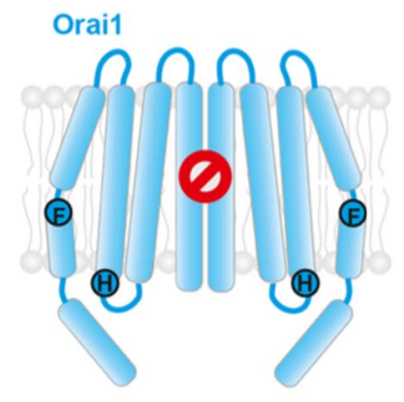

D "Brake" Orai1 + STIM1

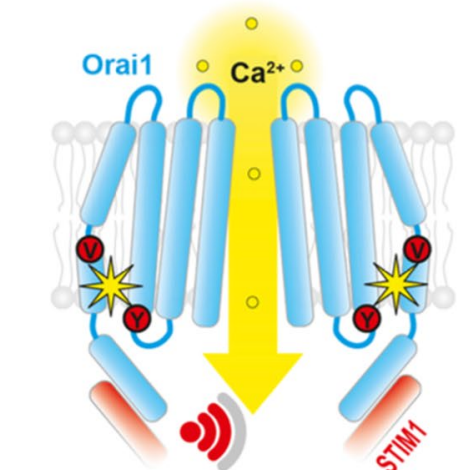

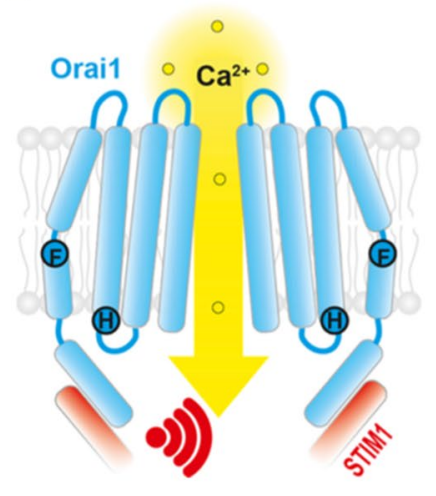

E "Brake" Orai1 + ANSGA

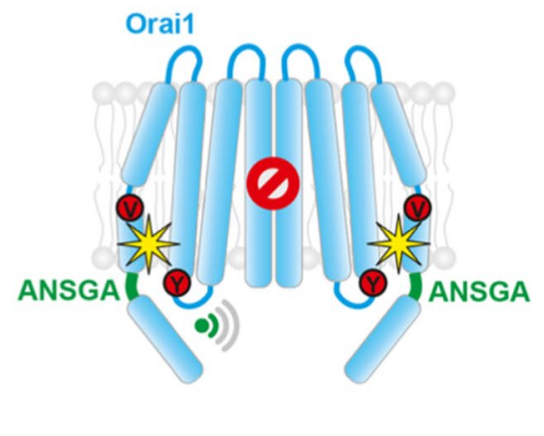

C WT Orai1 + ANSGA

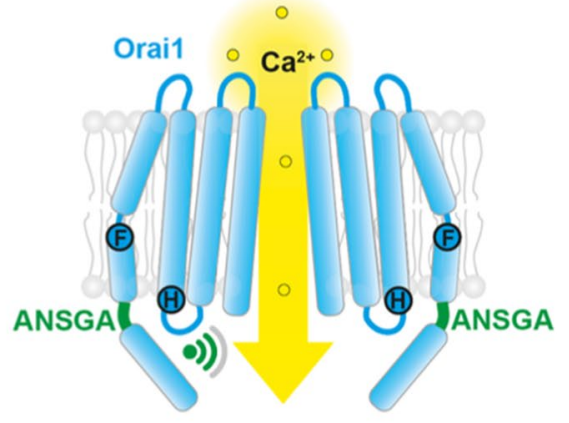

Figure 11. Hypothetical mechanism of h01channel activation by STIM1 and the "ANSGA" nexus mutation.

(A) h01 in a closed, quiescent state without any gating stimulus. (B) STIM1 binds the C-terminal extension (TM4ext) of h01, and exerts a strong gating signal that propagates through TM3-TM4 contacts to open the central pore. (C) The ANSGA mutation exerts a moderate gating signal that is able to open the h01 channel through intact TM3TM4 contacts. (D) Brake mutations at positions H171 and F246 introduced in h01 interfere with the gating signal by weakening the TM3-TM4 contacts, nevertheless, the stimulus exerted by STIM1 binding is still strong enough to open the channel. (E) Brake mutations attenuate the gating signal by ANSGA sufficiently enough to hinder the opening of the Orai channel.

We envision that our results on the various Orai channel isoforms could serve as a source of information for generated using the Drosophila TM4 mutant P288L corresponding to h01-P245L and the Drosophila TM2 mutant H206A corresponding to hO1-H134A (Hou et al., 2018; Hou et al., 2020; Liu et al., 2019). Our study indicates that mouse O1 ANSGA and Xenopus O1 ANSGA channels exhibit "intermediate-arrested" or "fully-activated" states that could be used for future structural work to gain further insights into hO1 channel activation and pharmacological inhibition. Several potent inhibitors of human Orai channels have been reported in the literature 
bioRxiv preprint doi: https://doi.org/10.1101/2021.10.26.465861; this version posted October 26, 2021. The copyright holder for this preprint (which was not certified by peer review) is the author/funder. All rights reserved. No reuse allowed without permission.

Augustynek et al.

Gating mechanisms of Orai calcium channels

608 specificity. Our work could help design strategies to generate Orai structures in complex with inhibitors, as a basis 609 of structure-based design of Orai subtype-specific channel modulators. 
Augustynek et al.

Gating mechanisms of Orai calcium channels

\section{Materials and Methods}

\section{Cloning and site-directed mutagenesis}

612

613

614

615

616

617

618

619

620

621

622

623

624

625

626

627

628

629

630

631

632

633

634

635

636

Xenopus Orai1 cDNA (Clone ID: 4633914, MXL1736-202771770, Horizon Discovery Ltd.) after PCR amplification was subcloned into pmCherry-C1. Human Orai2 was amplified by PCR from CFP-Orai2 peCFP-C1 provided by Prof. Christoph Romanin, University of Linz, Austria and sub-cloned into pmCherry-C1. Mouse Orai1 WT was constructed by site-directed mutagenesis-mediated generation of a stop codon after the coding sequence of Orai1 in mouse WT Orai1-eGFP peGFP-N1 provided by Prof. Francisco Javier Martin-Romero, University of Extremadura, Spain. All the forward primers used for site-directed mutagenesis are mentioned in the key resources table and the reverse primers used were reverse complementary. Pfu Ultra DNA polymerase (600380, Agilent Technologies) was used for site-directed mutagenesis.

\section{CRISPR/Cas9-mediated generation and validation of STIM1/STIM2 double knockout HEK293 cells}

To generate STIM1/STIM2 double knockout HEK293 cells using CRISPR/Cas9 genome editing technique, we first knocked out STIM1 from HEK293 cells as described in (Butorac et al., 2019). Briefly, two different guide RNA (gRNA) were designed using the Benchling CRISPR webtool (https://benchling.com/crispr) that target exon 1 of STIM1. The gRNA1 (5'-TTC TGT GCC CGC GGA GAC TC-3') was chosen to target a region encoding 5' untranslated region (UTR) of STIM1 and gRNA2 (5'-GTA TGC GTC CGT CTT GCC CTG-3') to target a region encoding the signal peptide of hSTIM1. Each of the gRNAs were cloned into pX330.pgkpuro vector (Harmsen, Klaasen, van de Vrugt, \& Te Riele, 2018) and co-transfected into the HEK293 (ATCC ${ }^{\circledR}$ CRL-1573 ${ }^{\text {TM }}$ ) cells. The transfected cells were selected by $96 \mathrm{~h}$ treatment with $1.5 \mu \mathrm{g} / \mathrm{ml}$ puromycin. The puromycin selected HEK293 cells were then used to knock out STIM2. STIM2 gRNA1 (5'-CGG AAC CAAT GAA CGC AGC C-3') was chosen to target a region that is thought to encode an alternate longer signal peptide of hSTIM2 (Graham, Dziadek, \& Johnstone, 2011), whereas gRNA2 (5'-GCT GGT AGC CGG AGC GGC GGA-3') was chosen to target the regular short signal peptide encoding sequence of hSTIM2 (Bhardwaj, Hediger, \& Demaurex, 2016). STIM2 gRNA1 was cloned into Bbsl-HF linearized pX330.pgkpuro and gRNA2 was cloned into Bbsl-HF linearized pU6-(Bbsl)_CBhCas9-T2A-mCherry (Addgene Plasmid \#64324). After co-transfection with STIM2 gRNA1 and gRNA2 plasmids the cells were sorted by Fluorescence-Activated Cell Sorting (FACS) with mCherry fluorescence and single cells were seeded in a 96-well plate. 
Augustynek et al.

Gating mechanisms of Orai calcium channels

637 To validate the knockout, genomic DNA was extracted from the resulting single-cell-derived clones and 5'-CCT TCC GCA GGG GTG TAG T-3' forward and 5'-CTC CAA CAG CCA AAG GTC AA-3' reverse primers were used to PCR amplify a 181 bp STIM1 amplicon. Similarly, 5'-CGG AAC CAA TGA ACG CAG C-3' forward and 5'-GAG TCG AGG CGG GAT GAA G-3' reverse primers were used to obtain a 222 bp STIM2 PCR product. Western blotting was performed as described in (Bhardwaj et al., 2020). Guinea pig polyclonal hSTIM1 antibody (Ercan, Chung, Bhardwaj, \& Seedorf, 2012) was used in a 1:1,000 dilution and peroxidase-conjugated affinipure goat antiguinea pig secondary antibody (106-035-003, Jackson Immuno Research) was used in a 1:10,000 dilution. Rabbit polyclonal hSTIM2 antibody (4917S, Cell Signaling) was used in a 1:1000 dilution and horseradish peroxidase (HRP)-conjugated goat anti-rabbit secondary lgG (W401B, Promega) was used in a 1:20,000 dilution. Mouse monoclonal Tubulin antibody (T9028, Sigma-Aldrich) was used in a 1:2,000 dilution and HRP-conjugated goat anti-mouse secondary antibody was used in a 1:3,000 dilution (172-1011, Bio-Rad). Following gDNA based PCR screening and Western blotting, SOCE recordings were performed using the S1/S2 DKO clones and Clone F2 was selected for further experiments.

\section{FLIPR assay to measure constitutive $\mathrm{Ca}^{2+}$ entry}

HEK293T (ATCC ${ }^{\circledR}$ CRL-3216 ${ }^{\mathrm{TM}}$ ) and S1/S2 DKO HEK293 cells were maintained in cell culture in 1X DMEM MEM non-essential amino acids and 1\% Penicillin-Streptomycin. The FLIPR assay to measure the constitutive $\mathrm{Ca}^{2+}$ entry was adapted from our previous study (Bulla et al., 2019). Briefly, the cells were seeded in Corning® 96well black polystyrene clear bottom microplates (CLS3603, Sigma-Aldrich) at a density of 30,000 in $100 \mu \mathrm{l}$ medium per well. The medium contained $\sim 0.2 \mathrm{mM} \mathrm{CaCl}_{2}$ to prevent excessive constitutive $\mathrm{Ca}^{2+}$ entry after transfection of constitutively active constructs. Since FBS is estimated to contain 3.5-4 $\mathrm{mM} \mathrm{Ca}^{2+}$ (Blankenship \& Heitman, 2005), low $\mathrm{Ca}^{2+}(\sim 0.2 \mathrm{mM})$ containing medium was formulated by supplementing the $\mathrm{Ca}^{2+}$ free $\mathrm{DMEM}(21068028$, Thermo Fisher Scientific) with 6\% FBS. Other supplements were $1 \mathrm{mM}$ sodium pyruvate, $10 \mathrm{mM}$ HEPES and 1X MEM non-essential amino acids. A total of $200 \mathrm{ng}$ plasmid DNA was transfected per well using Lipofectamine ${ }^{\circledR} 2000$ (Thermo Fisher Scientific). The growth medium of the cells was removed $16-20 \mathrm{~h}$ after transfection and the cells were loaded with $50 \mu$ of Calcium 5 indicator (FLIPR ${ }^{\circledR}$ Calcium 5 assay kit, R8186, Molecular Devices) prepared in modified Krebs buffer containing $0.2 \mathrm{mM} \mathrm{CaCl}_{2}, 1 \mathrm{mM} \mathrm{MgCl}_{2}, 140 \mathrm{mM} \mathrm{NaCl}, 4.8 \mathrm{mM} \mathrm{KCl}, 10 \mathrm{mM}$ D-glucose and 
Augustynek et al.

Gating mechanisms of Orai calcium channels

665

$495 \mathrm{~nm}$ LED module of the FLIPR, and the emitted fluorescence signal was filtered with a 515-575 nm emission filter. After recording a $50 \mathrm{~s}$ baseline, $50 \mu \mathrm{l}$ of $3.8 \mathrm{mM} \mathrm{CaCl}_{2}$-containing Krebs buffer was administered to the cells, resulting in $2 \mathrm{mM}$ final concentration of $\mathrm{Ca}^{2+}$. The changes in fluorescence intensity were measured for first $60 \mathrm{~s}$ after $\mathrm{CaCl}_{2}$ administration with an acquisition rate of $2 \mathrm{~Hz}$ and for further $180 \mathrm{~s}$ with $0.45 \mathrm{~Hz}$. The fluorescence signals were analyzed using the FLIPR Tetra software, ScreenWorks 3.1.1.8 (Molecular Devices). To quantify the constitutive $\mathrm{Ca}^{2+}$ entry dedicated to each mutant, a ratio of area under the curve (AUC) of the $\mathrm{Ca}^{2+}$ entry traces of the mutant Orai to that of wild type was calculated.

\section{Electrophysiology}

Whole-cell patch-clamp experiments were specifically designed to measure two distinct types of inward $\mathrm{Ca}^{2+}$ currents:

i) Canonical ICRAC evoked by $\mathrm{IP}_{3}$ and facilitated by interacting hO1 and hSTIM1 molecules.

ii) Constitutive $\mathrm{Ca}^{2+}$ influx mediated by the selected constitutively active hO1 mutants in the absence of hSTIM1 and IP 3 .

\section{ICRAC assay}

The experimental procedure was described in detail previously (Bhardwaj et al., 2020). Briefly, HEK293 S1/S2 DKO cells were trypsinized and seeded into the 6-well plate. When cells reached confluency of approx. $70-80 \%$, they were transiently co-transfected with plasmids encoding WT hO1 and WT STIM1. $5 \mu$ L Lipofectamine ${ }^{\circledR} 2000$ (Thermo Fisher Scientific) and $2+2 \mu \mathrm{g}$ of plasmid DNA encoding the proteins were mixed in Opti-MEM ${ }^{\mathrm{TM}}$ (Thermo Fisher Scientific) and applied onto the cells in a single well. After $6 \mathrm{~h}$ of incubation with the Lipofectamine-DNA complexes, cells were trypsinized and reseeded sparsely into the patch-clamp-compatible $35 \times 10 \mathrm{~mm}$ cell culture petri dishes.

ICRAC was measured after further $18 \mathrm{~h}$ of incubation at $37^{\circ} \mathrm{C}$ in humidified $5 \% \mathrm{CO}_{2}$ atmosphere $(24 \mathrm{~h}$ in total after transfection). Only cells exhibiting comparable (modest) fluorescence levels of both mCherry-Orai1 and GFPSTIM1 were selected for recordings. The experiments were performed at room temperature, in whole-cell configuration. Pipettes were pulled from $1.5 \mathrm{~mm}$ thin-wall borosilicate glass capillaries with filament (BF150-867.5, Sutter Instruments) using a horizontal P-1000 puller (Sutter Instruments) to obtain a serial resistance of around 
Augustynek et al.

Gating mechanisms of Orai calcium channels

691

692

693

694

695

696

697

698

699

700

701

702

703

704

705

706

707

708

709

710

711

712

713

714

715

716

2.5 M . Currents were recorded with PatchMaster software (HEKA Elektronik), using an EPC-10 USB amplifier (HEKA Elektronik). Upon establishment of giga seal and successful break-in into the single, mCherry fluorescent cell, $50 \mathrm{~ms}$ voltage ramps spanning -150 to $+150 \mathrm{mV}$ were delivered from a holding potential of $0 \mathrm{mV}$ every 2 seconds. Currents were filtered at $2.9 \mathrm{kHz}$ and digitized. Liquid junction potential was $10 \mathrm{mV}$ and currents were determined and corrected before each voltage ramp. Leak currents were corrected by subtracting the initial ramp currents from all subsequent currents using FitMaster software (HEKA Elektronik). Currents were extracted at -80 and $+80 \mathrm{mV}$ and normalized to cell capacity (size).

At break in, the bath solution contained $120 \mathrm{mM} \mathrm{NaCl}, 10 \mathrm{mM}$ tetraethylammonium chloride (TEA-Cl), $2 \mathrm{mM} \mathrm{MgCl}$, $10 \mathrm{mM} \mathrm{CaCl}$, $10 \mathrm{mM}$ HEPES, and $32 \mathrm{mM}$ glucose $(\mathrm{pH} 7.2$ with $\mathrm{NaOH}, 300 \mathrm{mOsmol})$. After 60 seconds of recording, cells were perfused with nominal $\mathrm{Ca}^{2+}$-free bath solution (the same as above, but without $10 \mathrm{mM} \mathrm{CaCl}_{2}$, osmolarity was adjusted with more glucose).

Calcium-free internal solution contained 120 mM Cs-glutamate, 3 mM MgCl , 10 mM HEPES, 0.05 mM D-myoinositol 1,4,5-trisphosphate, trisodium salt ( $\left.\mathrm{IP}_{3}\right)$ (407137, Calbiochem) and $20 \mathrm{mM} \mathrm{EGTA}(\mathrm{pH} 7.2$ with CsOH, 310 mOsmol with glucose).

\section{Constitutive $\mathrm{Ca}^{2+}$ influx assay}

This experimental approach was largely identical to the ICRAC assay, with two significant modifications:

i) HEK293 S1/S2 DKO cells were transfected each time only with a single plasmid encoding the relevant hO1 mutant. Unlike in the previous assay, no STIM1 was co-expressed.

ii) Calcium-free internal solution contained $120 \mathrm{mM} \mathrm{Cs}$-glutamate, $3 \mathrm{mM} \mathrm{MgCl}, 10 \mathrm{mM}$ HEPES and 20 mM BAPTA (B1212, Invitrogen), not EGTA (pH 7.2 with $\mathrm{CsOH}, 310$ mOsmol adjusted with glucose). No $\mathrm{IP}_{3}$ was added to the solution.

\section{NFAT1 translocation assay}

Fluorescence microscopy and confocal NFAT subcellular localization studies were performed as described earlier (Schober et al., 2019). ImageJ was used to analyze subcellular NFAT localization by intensity measurements of the cytosol and the nucleus (nucleus/cytosol ratios: inactive $(<0.85)$, homogenous $(0.85-1.15)$, and active $(>1.15)$ ). All represented images of Orai isoforms as well as NFAT localization were created with a custom-made software 
Augustynek et al.

Gating mechanisms of Orai calcium channels

717

718

719

720

721

722

723

724

725

726

727

728

729

730

731

732

733

734

735

736

737

738

739

740

741

742

integrated into MATLAB (v7.11.0, The MathWorks, Inc.). The experiments were performed on three different days at room temperature and the resulting data of all experiments are presented as mean \pm S.D. (standard deviation) for the indicated number of experiments.

\section{Cell Surface Biotinylation}

Cell surface biotinylation was performed as described (Simonin \& Fuster, 2010). Cells were rinsed with 1x PBS and surface proteins were biotinylated by incubating cells with $1.5 \mathrm{mg} / \mathrm{ml}$ sulfo-NHS-LC-biotin in $10 \mathrm{mM}$ triethanolamine ( $\mathrm{pH} 7.4), 1 \mathrm{mM} \mathrm{MgCl}_{2}, 2 \mathrm{mM} \mathrm{CaCl}_{2}$, and $150 \mathrm{mM} \mathrm{NaCl}$ for 90 min with horizontal motion at $4^{\circ} \mathrm{C}$. After labeling, plates were washed with quenching buffer (PBS containing $1 \mathrm{mM} \mathrm{MgCl}_{2}, 0.1 \mathrm{mM} \mathrm{CaCl}$, and 100 $\mathrm{mM}$ glycine) for $20 \mathrm{~min}$ at $4^{\circ} \mathrm{C}$, then rinsed once with $1 \mathrm{X}$ PBS. Cells were then lysed in RIPA buffer [150 mM NaCl, $50 \mathrm{mM}$ Tris $\cdot \mathrm{HCl}(\mathrm{pH} 7.4), 5 \mathrm{mM}$ EDTA, $1 \%$ Triton X-100, 0.5\% deoxycholate, and $0.1 \%$ SDS] and lysates were cleared by centrifugation. Cell lysates of equivalent amounts of protein were equilibrated overnight with streptavidin agarose beads at $4^{\circ} \mathrm{C}$. Beads were washed sequentially with solutions $\mathrm{A}[50 \mathrm{mM} \mathrm{Tris} \cdot \mathrm{HCl}(\mathrm{pH} 7.4), 100 \mathrm{mM} \mathrm{NaCl}$, and $5 \mathrm{mM}$ EDTA] three times, $\mathrm{B}[50 \mathrm{mM}$ Tris $\cdot \mathrm{HCl}(\mathrm{pH} 7.4)$ and $500 \mathrm{mM} \mathrm{NaCl}]$ two times, and $\mathrm{C}(50 \mathrm{mM}$ Tris $\cdot \mathrm{HCl}$, $\mathrm{pH}$ 7.4) once. Biotinylated proteins were then released by heating to $95^{\circ} \mathrm{C}$ with $2.5 \mathrm{X}$ Lämmli buffer.

\section{Structural modeling}

The structural model of hO1 was generated using Modeller 9.21 (Fiser, Do, \& Sali, 2000; Sali \& Blundell, 1993; Webb \& Sali, 2016) based on the closed-latched structure (PDB ID: 4HKR (Hou et al., 2012)), downloaded from the Orientations of Proteins in Membranes (OPM) database (Lomize, Lomize, Pogozheva, \& Mosberg, 2006). Sequences of the template and query proteins were aligned using ClustalW 2.1 (Larkin et al., 2007). During model optimization, main chain atoms of residues $72-106,121-143$, and $235-291$, roughly corresponding to the TM regions, were kept fixed in order to avoid large conformational changes. Six-fold symmetry constraints were introduced for main chain atoms of residues 99-128, 138-178, and 190-242, corresponding to loop regions and flanking TM regions. Based on PSIPRED 4.0 (Buchan, Minneci, Nugent, Bryson, \& Jones, 2013; Jones, 1999), ahelical constraints were introduced on residues 117-154, 164-199, and 231-286. Loop modeling protocol was applied on residues 107-120, 144-164, and 198-234. In total 50 models were built, and 10 loop modeling tries were attempted on each model. The final model was chosen based on lowest objective function values as reported 
Augustynek et al.

Gating mechanisms of Orai calcium channels

by Modeller for loop modeling, and visual inspection to avoid protein loops protruding into the anticipated position of the membrane bilayer.

\section{Molecular dynamics (MD) simulations}

Simulation systems were prepared and mutations were introduced using CHARMM-GUI (Brooks et al., 2009; Jo et al., 2014; Jo, Kim, \& Im, 2007; Jo, Kim, Iyer, \& Im, 2008; Jo, Lim, Klauda, \& Im, 2009; Lee et al., 2016; Wu et al., 2014), E190 was protonated, and truncated termini were acetylated/methylated. Two $\mathrm{Ca}^{2+}$ ions were introduced in the structure, one at the position determined by X-ray crystallography in the structure (PDB ID: 4HKR), and another $5 \AA$ below along the axis of the pore. The protein was surrounded by palmitoyl-oleoyl-phosphatidylcholine (POPC) lipids in a rectangular box with size of $120.12 \times 120.12 \times 129.76 \AA$ and solvated in TIP3 water with neutralizing ions and $150 \mathrm{mM} \mathrm{NaCl}$. The simulations were performed using GPU-accelerated pmemd of AMBER 18 (Case et al., 2018) using the CHARMM36m force-field (Huang et al., 2017), under NPYT conditions, 1 bar pressure, constant surface tension of zero and a temperature of $310 \mathrm{~K}$. Equilibration of simulation systems was performed as per the protocol prescribed by CHARMM-GUI. Motions of protein variants were simulated for $200 \mathrm{~ns}$ for 5 independent replicas each.

\section{Analysis of MD trajectories}

758 Contact analysis was performed using the analysis.distances.contact_matrix function of MDAnalysis (MichaudAgrawal, Denning, Woolf, \& Beckstein, 2011) (Gowers et al., 2016) on all atoms of residues, with a cut-off range of $5 \AA$. Inter- and intra-subunit atomic contacts in the Orai hexamer have been distinguished and counted separately. Residues were taken to be in contact if any pair of their atoms were in contact. Helicity analysis was performed by custom scripts based on MDAnalysis to calculate for each residue $i$ the angle $\alpha_{i}=\psi_{i}+\varphi_{i+1}$, where $\varphi_{i}$ and $\psi_{i}$ are the Ramachandran-angles for residue $i$. For residues in a perfect $\alpha$-helix, the value of $\alpha=-105^{\circ}$. During helicity analysis, odd (A, C, E chains with a more extended TM4-TM4ext region) and even (B, D, F chains with a helix-turn-helix/backfolded TM4-TM4ext region) subunits of the Orai hexamer were distinguished owing to the 3-fold symmetry of the starting structure. Pore diameter analysis was performed by HOLE using elements of the programming interface from MDAnalysis (Smart, Goodfellow, \& Wallace, 1993; Smart, Neduvelil, Wang, Wallace, \& Sansom, 1996). Pore diameters were calculated every ns of the simulations and averaged over all frames. 
bioRxiv preprint doi: https://doi.org/10.1101/2021.10.26.465861; this version posted October 26, 2021. The copyright holder for this preprint (which was not certified by peer review) is the author/funder. All rights reserved. No reuse allowed without permission.

Augustynek et al.

Gating mechanisms of Orai calcium channels

Acknowledgements

771 Calculations were performed on UBELIX (http://www.id.unibe.ch/hpc), the HPC cluster at the University of Bern.

772 We would like to thank Dr. Stefan Mueller, Thomas Schaffer and Bernadette Nyfeler at the FACS facility, Institute 773 of Pathology, University of Bern for their help in FACS sorting of CRISPR/Cas9-generated knockout cells. Also, 774 we thank Tamara Locher, University of Bern for her technical assistance in cell culture. We thank Prof. Francisco 775 Javier Martin-Romero, University of Extremadura, Spain for providing us the mO1-eGFP construct, Dr. Matthias 776 Seedorf, Heidelberg University, Germany for providing us the mCherry-tagged hO1 and hO3 constructs and Prof. 777 Christoph Romanin, University of Linz, Austria for providing us the CFP-hO2 construct. We are thankful to Dr. 778 Anant B. Parekh, National Institute of Environmental Health Sciences, North Carolina, USA for providing important 779 feedback on our manuscript. H.G. thanks Austrian Science Fund (FWF) for PhD scholarship through W1250 780 NanoCell PhD program. I.F. was funded by FWF project P32075.-B. B.A., G.G., M.A.H. and R.B. were funded by 781 the Swiss National Science Foundation Sinergia grants (CRS115_180326 and CRS113_160782). R.B. was also supported by the Marie Curie Actions International Fellowship Program IFP TransCure, University of Bern,

783 Switzerland (from 2014 to 2017).

784 Additional information

$785 \quad$ Funding

\begin{tabular}{lll} 
Funder & Grant reference number & Author \\
\hline Swiss National Science Foundation & CRS115_180326 & Matthias A. Hediger \\
\hline Swiss National Science Foundation & CRS113_160782 & Matthias A. Hediger \\
\hline Austrian Science Fund & P32075.-B & Irene Frischauf \\
\hline $\begin{array}{l}\text { The funders had no role in study design, data collection and interpretation, or the decision to submit } \\
\text { the work for publication. }\end{array}$
\end{tabular}


bioRxiv preprint doi: https://doi.org/10.1101/2021.10.26.465861; this version posted October 26, 2021. The copyright holder for this preprint (which was not certified by peer review) is the author/funder. All rights reserved. No reuse allowed without permission.

Augustynek et al.

Gating mechanisms of Orai calcium channels

787

788

789

790

791

792

793

794

795

796

797

798

799

800

801

802

803

804

805

806

807

808

\section{Author contributions}

Bartlomiej Augustynek, Electrophysiology Experimentation, Data Analysis and Curation, Writing - original draft, Writing - review and editing; Gergely Gyimesi, Homology modeling, Molecular dynamics simulations, Data Analysis, Writing - original draft, Writing - review and editing; Jan Dernič, FLIPR Experimentation; Matthias Sallinger, NFAT1 translocation Experimentation; Giuseppe Albano, Cell surface biotinylation Experimentation; Gabriel Jonathan Klesse, Site-directed mutagenesis; Palanivel Kandasamy, CRISPR/Cas9 knockout generation; Herwig Grabmayr, NFAT1 translocation Experimentation; Irene Frischauf, Writing - original draft, Writing - review and editing; Daniel G. Fuster, Supervision, Methodology, Writing - review and editing; Christine Peinelt, Supervision, Methodology, Writing - review and editing; Matthias A. Hediger, Project Strategy, Project Coordination and Supervision, Manuscript Writing; Rajesh Bhardwaj, Conceptualization, Supervision, Validation, Investigation, Site-directed mutagenesis and FLIPR Experimentation, CRISPR/Cas9 knockout generation, Data Analysis and Curation, Visualization, Methodology, Writing - original draft, Project administration, Writing - review and editing.

\section{Author ORCIDs}

Bartlomiej Augustynek https://orcid.org/0000-0002-4912-2936

Gergely Gyimesi https://orcid.org/0000-0002-2054-4633

Herwig Grabmayr https://orcid.org/0000-0003-0870-5833

Palanivel Kandasamy https://orcid.org/0000-0003-3795-2876

Irene Frischauf https://orcid.org/0000-0003-0661-2932

Daniel G. Fuster https://orcid.org/0000-0001-7220-1803

Christine Peinelt https://orcid.org/0000-0002-3474-6893

Matthias A. Hediger http://orcid.org/0000-0003-1946-027X

Rajesh Bhardwaj http://orcid.org/0000-0002-5599-487X 
Augustynek et al.

Gating mechanisms of Orai calcium channels

\section{Supplementary Figures}
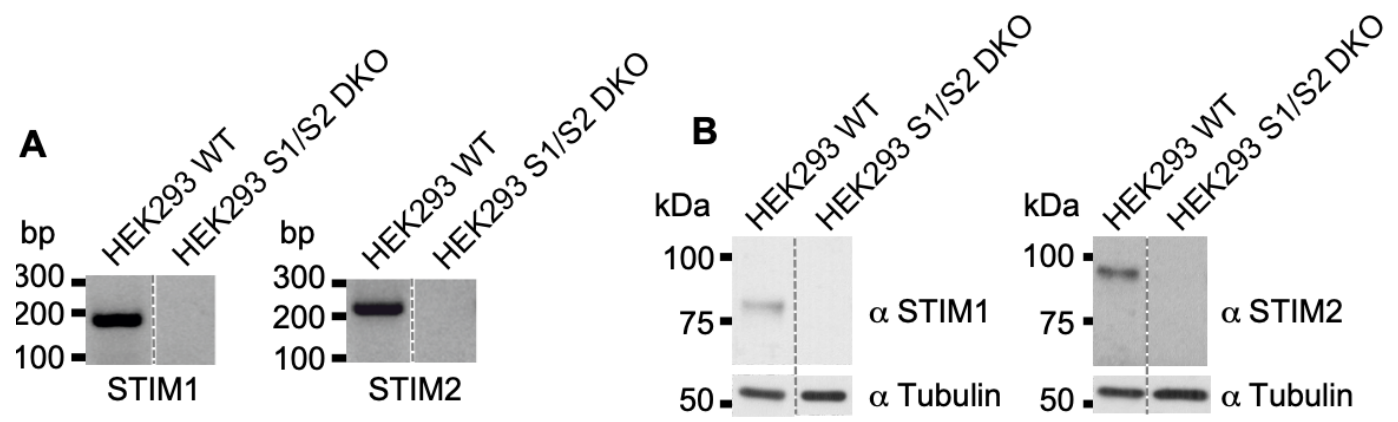

C

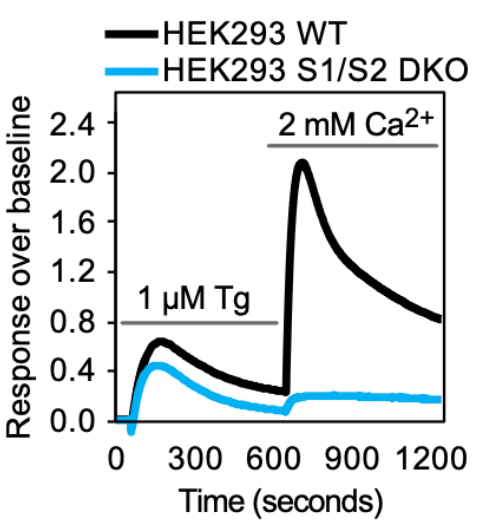

D

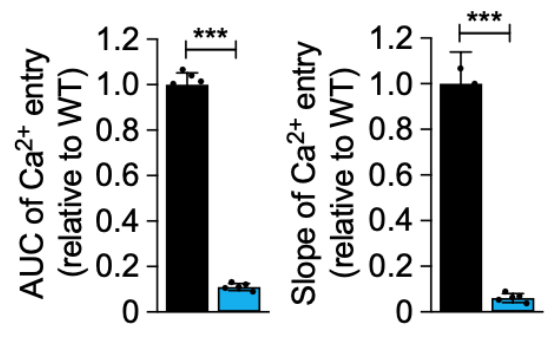

\section{Figure 2- figure supplement 1. Validation of HEK293 S1/S2 DKO cells.}

812 (A) Agarose gel electrophoresis showing PCR products of STIM1 and STIM2 using genomic DNA template isolated from HEK293 WT and 813 S1/S2 DKO cells. (B) Western blot analysis of HEK293 WT and S1/S2 DKO cells using human STIM1 and STIM2 antibodies. (C) 814 Representative SOCE measurement traces from HEK293 WT and S1/S2 DKO cells treated with $1 \mu \mathrm{M}$ thapsigargin $(\mathrm{Tg})$ in nominally 815 calcium free buffer followed by add-back of $2 \mathrm{mM} \mathrm{CaCl}_{2}$. (D) Quantifications of area under the curve (AUC) and slope of the $\mathrm{Ca}^{2+}$ entry 816 traces (second peak in "C") represented as mean $\pm S D$; $n=5$. 
Augustynek et al.

A

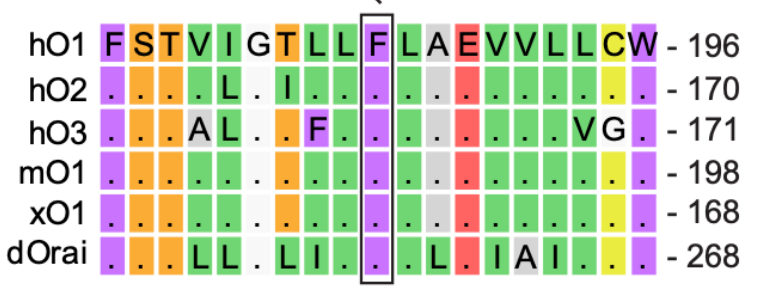

C

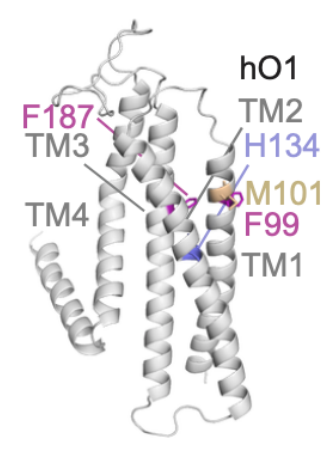

$\mathbf{F}$

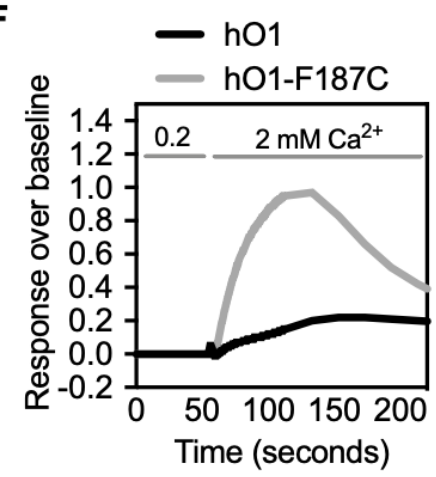

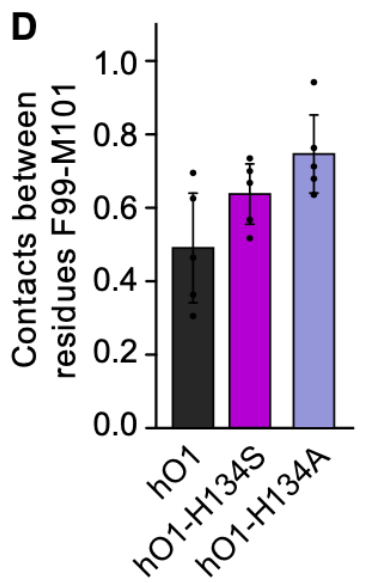

G

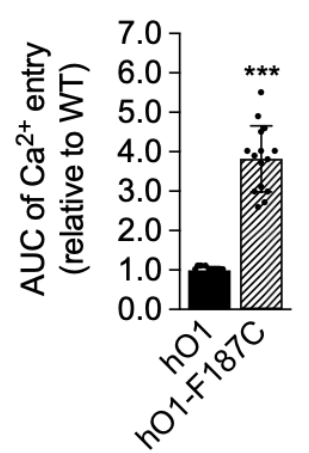

Gating mechanisms of Orai calcium channels
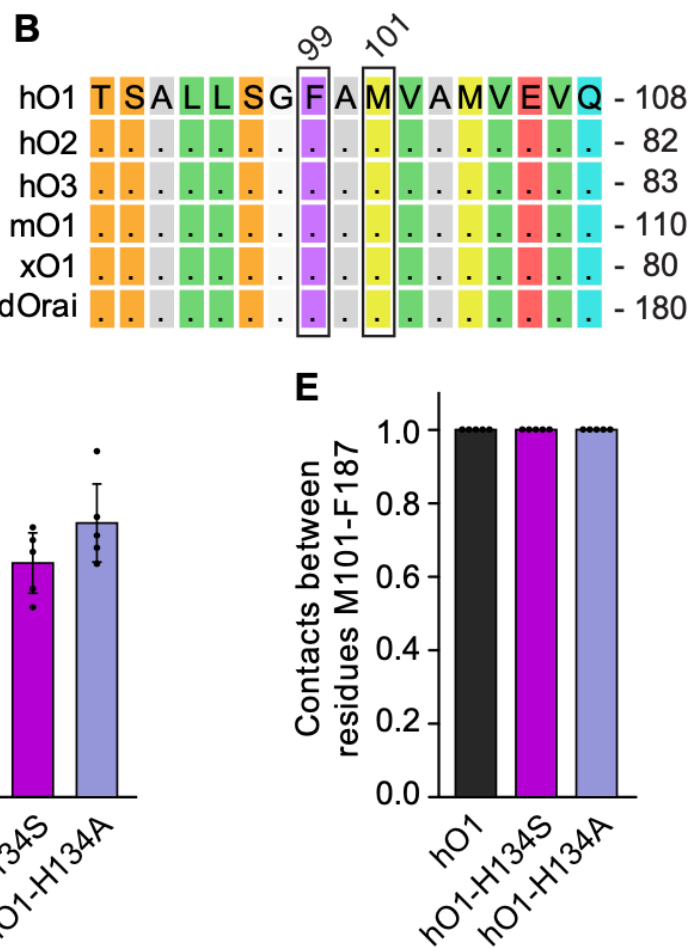

H

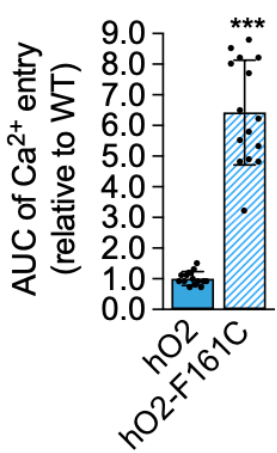

I

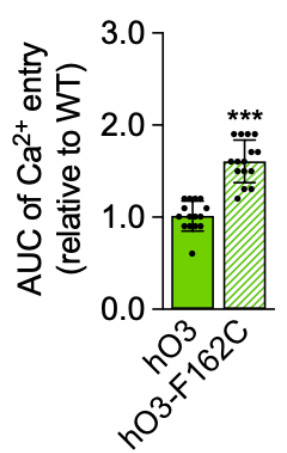

818 Figure 2- figure supplement 2. Inter-subunit F99-M101 contacts in h01 WT and H134 mutant, and constitutive activity of h01 TM3 819 F187C mutant and corresponding h02 and h03 mutants.

820 (A) Multiple sequence alignment of TM3 of h01, hO2, hO3, $\mathrm{m01}, \mathrm{xO1}$ and dOrai is shown, highlighting the conserved F187 residue. (B) 821 Multiple sequence alignment of TM1 of h01, hO2, hO3, m01, xO1 and dOrai is shown, highlighting the conserved F99 and M101 residue.

822 (C) Cartoon representation of a single subunit of h01 model depicting indicated residues. (D) Frequencies of contacts between F99 and 823 M101 in h01 WT, H134S and H134A mutant channels (mean \pm SD; n=5). (E) Contact frequencies between M101 and F187 in WT h01, 824 h01-H134S and h01-H134A mutant channels (mean \pm SD; $n=5$ ). (F) Representative constitutive $\mathrm{Ca}^{2+}$ entry traces of HEK293 cells 825 transfected with WT h01 and h01-F187C constructs with initial baseline recording in $0.2 \mathrm{mM} \mathrm{CaCl}$, followed by addition of $2 \mathrm{mM} \mathrm{CaCl}_{2}$. 826 The quantified AUC of $\mathrm{Ca}^{2+}$ entry peak from HEK293 cells expressing (G) WT h01 and h01-F187C, (H) WT hO2 and h02-F161C and (I) 827 WT hO3 and hO3-H162C (mean \pm SD; $n=15) . p \leq 0.001$ is indicated as “***”. 
bioRxiv preprint doi: https://doi.org/10.1101/2021.10.26.465861; this version posted October 26, 2021. The copyright holder for this preprint (which was not certified by peer review) is the author/funder. All rights reserved. No reuse allowed without permission.

Augustynek et al.

Gating mechanisms of Orai calcium channels

A

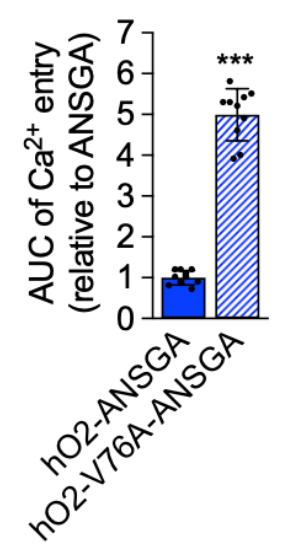

B

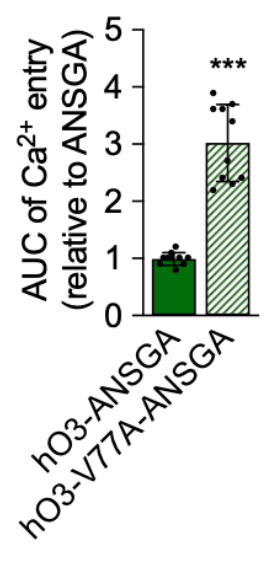

C

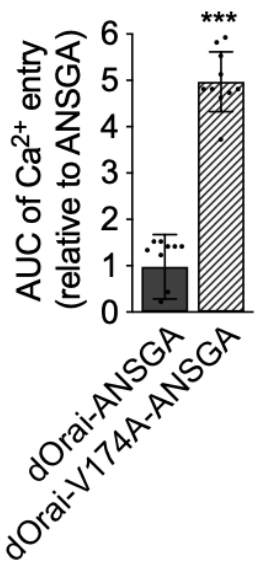

829 Figure 3- figure supplement 1. Insertion of pore hydrophobic gate (Val) mutations in the ANSGA mutants of hO2, h03 and dOrai 830 leads to their constitutive activation.

831 (A-C) AUC of the constitutive $\mathrm{Ca}^{2+}$ entry traces from indicated Orai mutants expressed in HEK293 S1/S2 DKO cells (mean $\pm \mathrm{SD} ; \mathrm{n}=10$ ). $p$ $832 \leq 0.001$ is indicated as "***". 
Augustynek et al.

Gating mechanisms of Orai calcium channels
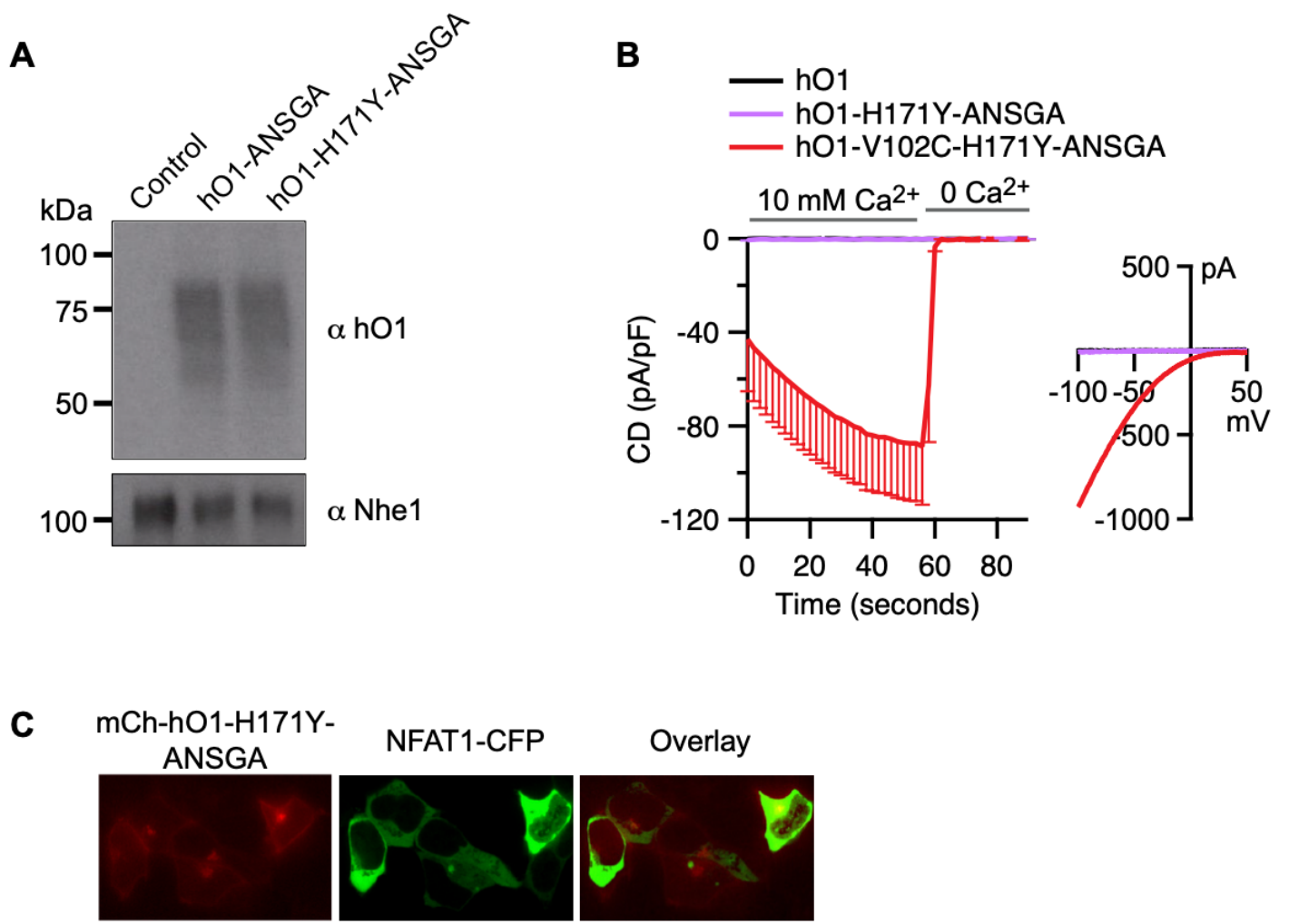

NFAT1-CFP

Overlay
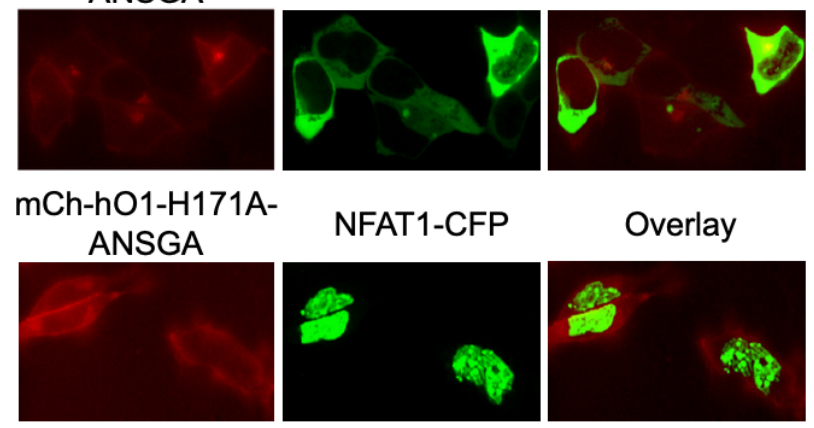

NFAT1-CFP

Overlay
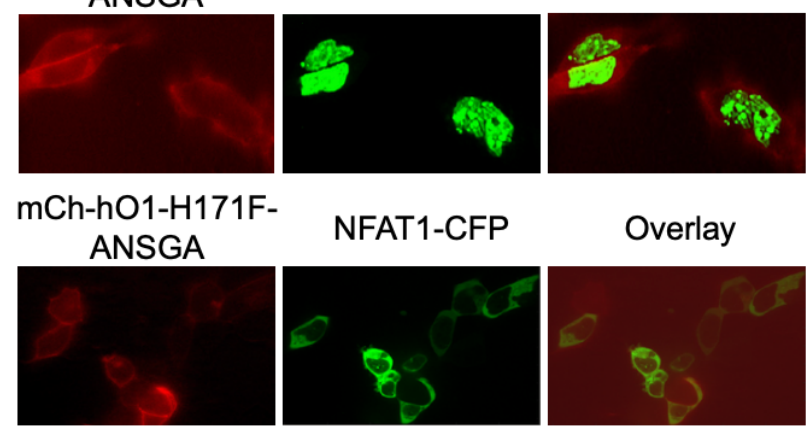

NFAT1-CFP

Overlay
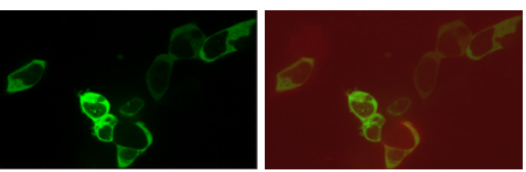

834 Figure 4- figure supplement 1. H171Y substitution inhibits the constitutive activity of h01-ANGSA channel without affecting the 835 plasma membrane localization and assembly of the channel.

836 (A) h01 immunoblot of surface biotinylated mCherry control, mCherry-h01-ANSGA and mCherry-h01-H171Y-ANSGA proteins expressed 837 in HEK293 S1/S2 DKO cells. Plasma membrane protein Nhe1 is shown as a loading control. (B) Current densities (CD) of the constitutive $838 \mathrm{Ca}^{2+}$ currents recorded from HEK293 S1/S2 DKO cells transiently overexpressing: WT h01 ( $\left.n=6\right)$, h01-H171Y-ANSGA ( $\left.n=8\right)$ and h01839 V102C-H171Y-ANSGA $(n=8)$, presented as average values, -SEM, with corresponding average current-voltage $(I / V)$ relations extracted at $840 \mathrm{t}=59 \mathrm{~s}$. (C) Representative confocal microscopy images of HEK293 cells co-expressing NFAT1-CFP with either mCherry-h01-H171Y841 ANSGA, h01-H171A-ANSGA or h01-H171F-ANSGA constructs along with the CFP/mCherry overlay images. 
Augustynek et al.

A

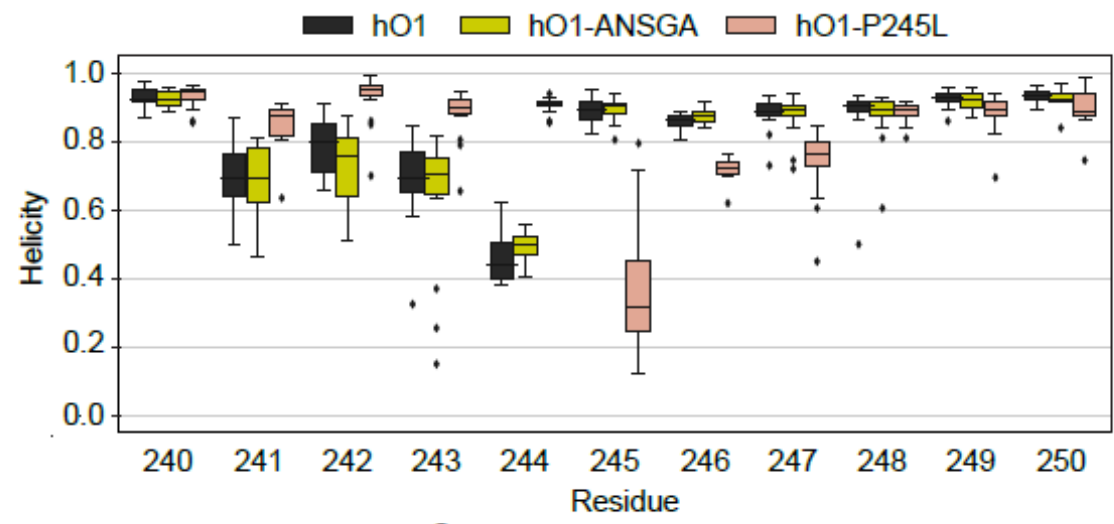

B

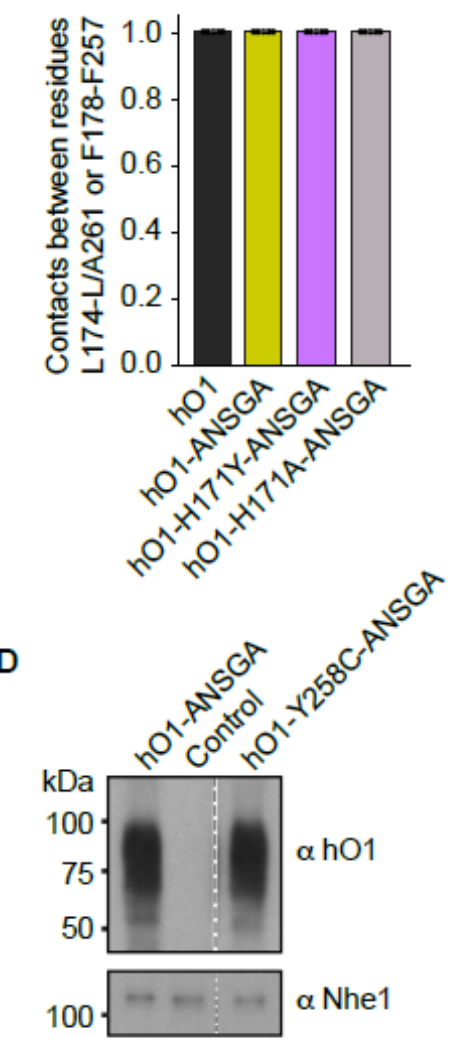

C
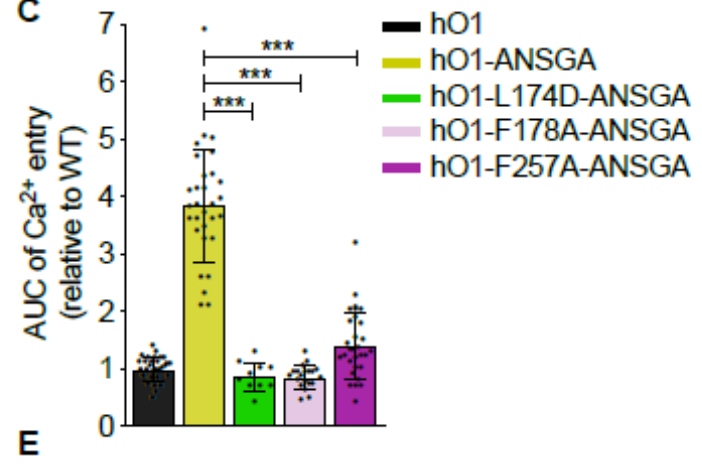

mCh-hO1-A175CANSGA

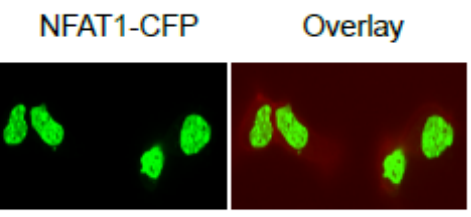

mCh-hO1-Y258CANSGA

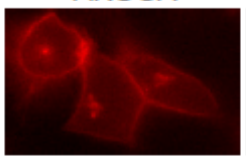

mCh-hO1-A175CY258C-ANSGA

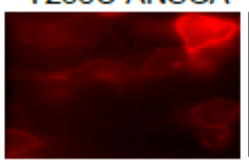

NFAT1-CFP

Overlay

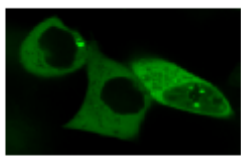

NFAT1-CFP

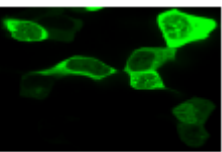

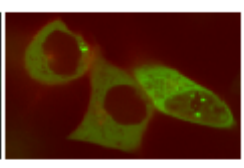

Overlay

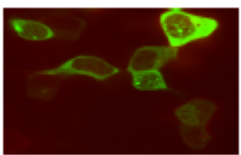

Figure 5- figure supplement 1. Relevance of selected TM3 and TM4 residues in the constitutive activity of h01-ANSGA channel. (A) Helicity of TM4 residues around position 245 of hO1 averaged over 5 MD trajectories for chains A, C, and E, giving 15 points per residue and system (see Methods). Box-and-whiskers plot showing quartiles is used, outliers are plotted individually. (B) Intra-subunit contact frequencies between residue pairs L174-L/A261 and F178-F257 averaged over each of the 5 MD trajectories are shown for various simulation systems. Data are identical for both residue pairs. (C) The AUC of constitutive $\mathrm{Ca}^{2+}$ entry recorded in HEK293 S1/S2 DKO cells expressing mCherry-tagged WT h01 or indicated ANSGA variants (mean $\pm S D ; n \geq 10$ ). $p \leq 0.001$ is indicated as "***". (D) h01 immunoblot of surface biotinylated mCherry control, mCherry-h01-ANSGA and mCherry-h01-Y258C-ANSGA proteins expressed in HEK293 S1/S2 DKO cells along with plasma membrane protein Nhe1 shown as a loading control. (E) Representative confocal microscopy images of HEK293 cells co-expressing NFAT1-CFP and either of the indicated mCherry-Orai-ANSGA constructs along with the CFP/mCherry overlay images. 
Augustynek et al.

Gating mechanisms of Orai calcium channels
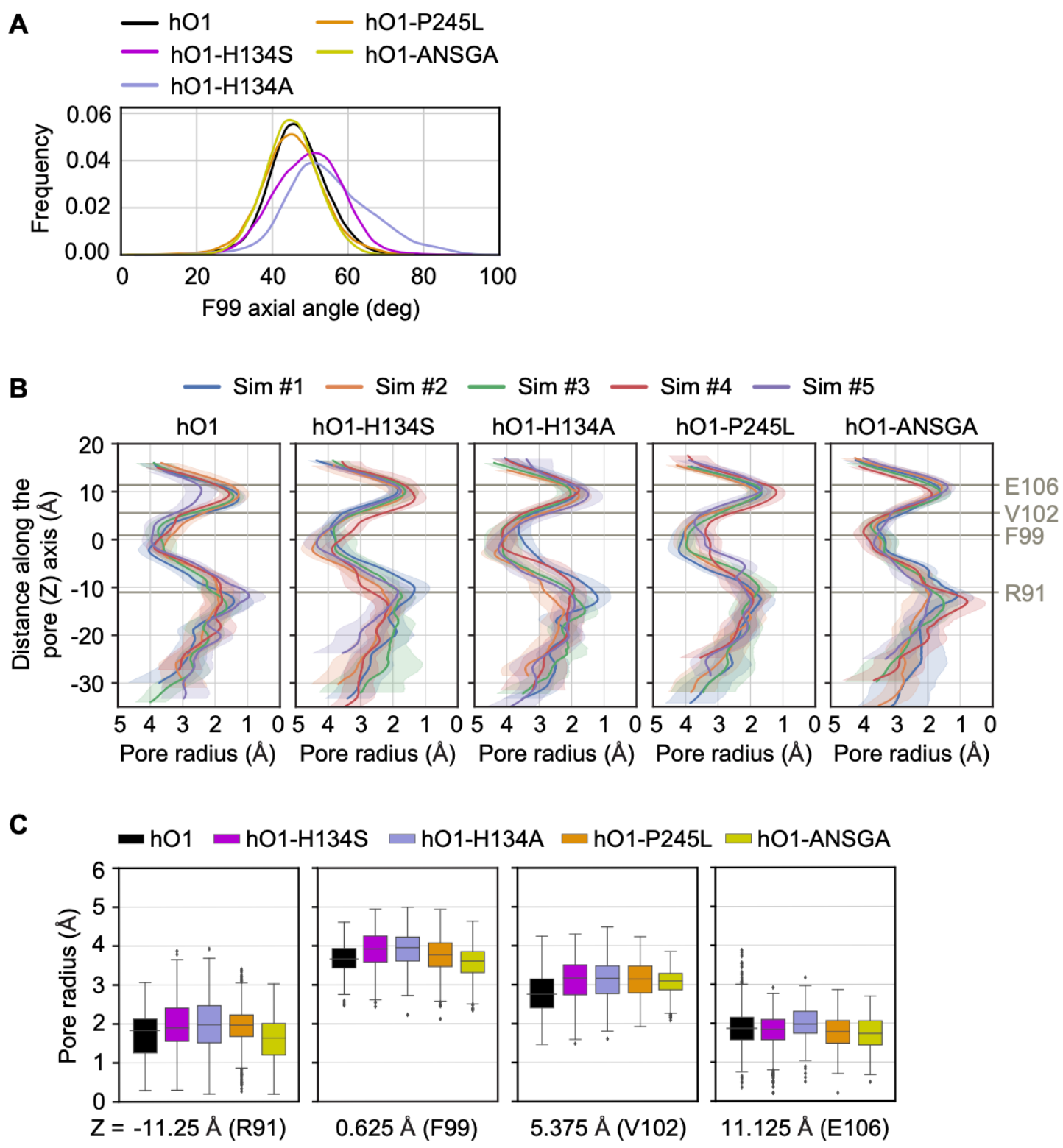

854 Figure 5- figure supplement 2. Impact of H134S, H134A, P245L and ANSGA mutations on TM1 rotation and pore dilation.

855 (A) Axial angle distribution of the F99 side-chain of h01 as defined by Yamashita et al., 2017 are shown (Yamashita et al., 2017) for WT 856 h01, h01-H134S, h01-H134A, h01-P245L and h01-ANSGA mutants. In loose terms, the axial angle measures the angle between the 857 pore axis, the center of mass of the $C_{a}$ atoms of residues $96-102$, and the $C_{a}$ atom of residue 99 . Values are averaged for all 5 MD 858 trajectories and for all six subunits of the Orai hexamer. (B) Pore radius as calculated by the HOLE program for each MD trajectory of 859 various simulation systems. Shaded regions show average and SD of pore radius. Residues of functional importance in TM1 are marked 860 for scale. (C) For comparison, values of pore radius along the trajectory of the Orai channel variants at cross-sections corresponding to the 861 positions of TM1 residues marked in panel B are shown. For each system and cross-section, pore radii for 200 frames from 5 simulations 862 (total 1000 points) are plotted using a box-and-whiskers plot showing quartiles and outliers. 
bioRxiv preprint doi: https://doi.org/10.1101/2021.10.26.465861; this version posted October 26, 2021. The copyright holder for this preprint (which was not certified by peer review) is the author/funder. All rights reserved. No reuse allowed without permission.

Augustynek et al.

Gating mechanisms of Orai calcium channels

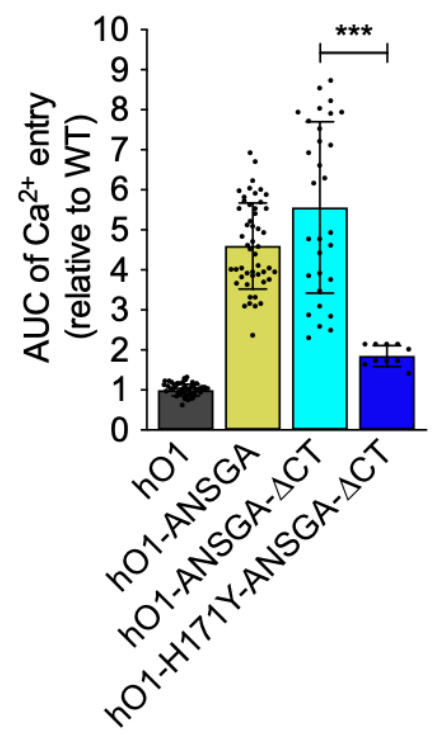

864 Figure 6- figure supplement 1. The TM4 extension beyond the ANSGA region of h01 is dispensable for H171Y mediated inhibition 865 of constitutive activity.

866 The quantified AUC of constitutive $\mathrm{Ca}^{2+}$ entry recorded in HEK293 S1/S2 DKO cells expressing the indicated h01-ANSGA mutants relative 867 to the WT h01 channel (mean $\pm S D ; n=10$ ). $p \leq 0.001$ is indicated as "***". 
Augustynek et al.

Gating mechanisms of Orai calcium channels

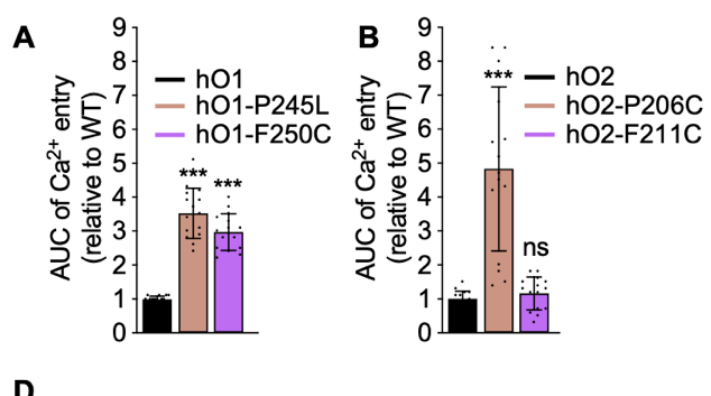

D
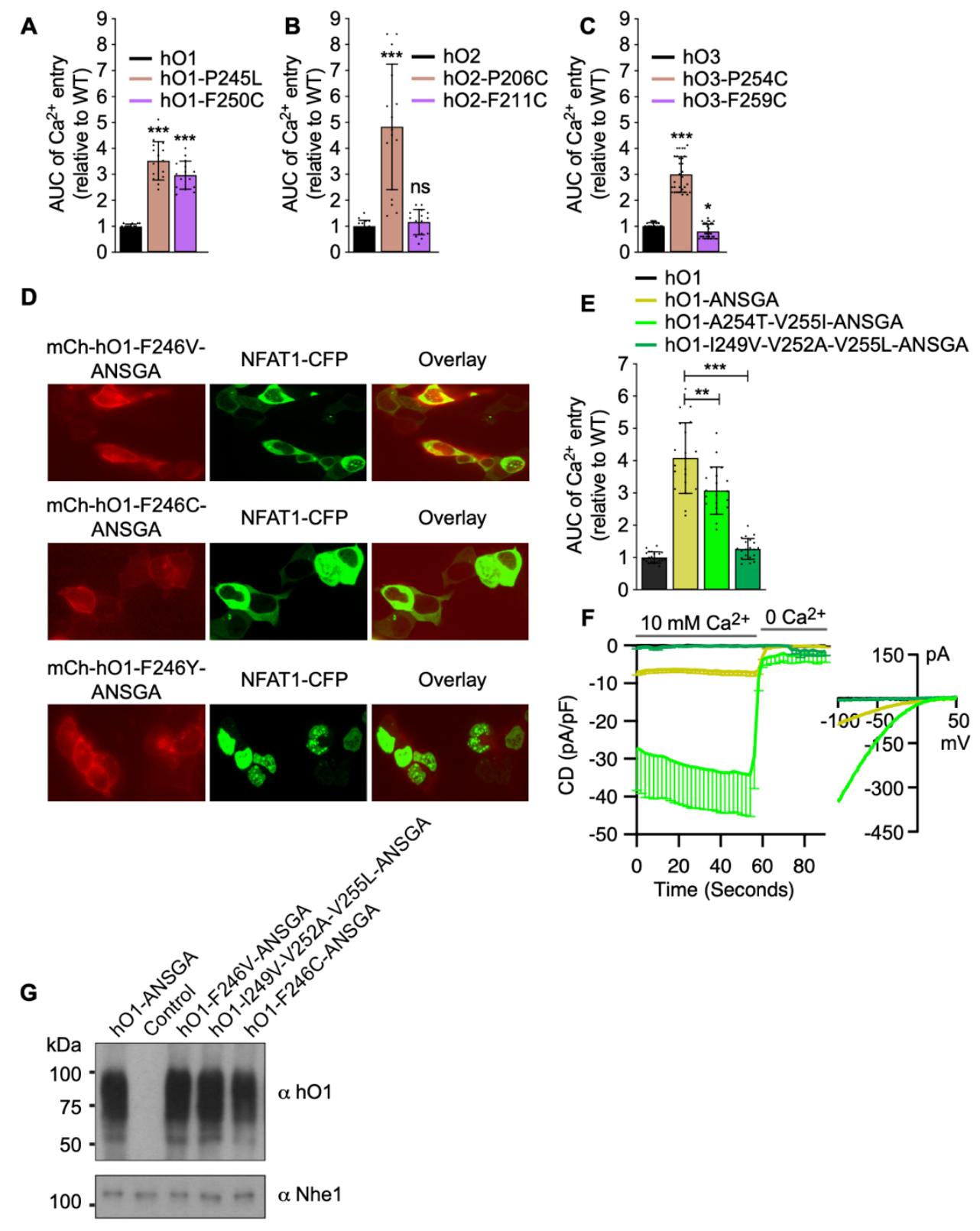

Figure 7- figure supplement 1.

870 The quantified AUC of constitutive $\mathrm{Ca}^{2+}$ entry recorded in HEK293 cells expressing (A) h01-P245L and h01-F250C, (B) hO2-P206C and 871 hO2-F211C and (C) hO3-P254C and h03-F259C relative to their respective WT Orais (mean \pm SD; $n \geq 15$ ). $p \leq 0.001$ is indicated as "***", $8720.01<p<0.05$ as "*” and $p \geq 0.05$ as "ns". (D) Representative confocal microscopy images of HEK293 cells co-expressing NFAT1-CFP 873 with either F246V, F246C or F246Y mutants of mCherry-h01-ANSGA along with the CFP/mCherry overlay images. (E) AUC quantifications of constitutive $\mathrm{Ca}^{2+}$ influx measured in HEK293 S1/S2 DKO cells expressing h01-ANSGA or its A254T-V255I and I249V-V252A-V255L mutants relative to WT h01 (mean $\pm \mathrm{SD} ; \mathrm{n}=20$ ). $p \leq 0.001$ is indicated as "***" and $0.001<p \leq 0.01$ as "**". (F) Current densities (CD) of the constitutive $\mathrm{Ca}^{2+}$ currents recorded from HEK293 S1/S2 DKO cells transiently overexpressing: WT h01 ( $\left.n=6\right)$, h01-ANSGA ( $\left.n=23\right)$, h01-A254T-V255I-ANSGA ( $n=8)$ and h01-I249V-V252A-V255L-ANSGA $(n=6)$, presented as average values, -SEM, with corresponding average current-voltage $(I / V)$ relations extracted at $t=59 \mathrm{~s}$. $(\mathbf{G})$ h01 immunoblot of surface biotinylated mCherry control, mCherry-h01ANSGA and other indicated ANSGA mutant proteins expressed in HEK293 S1/S2 DKO cells. Plasma membrane protein Nhe1 is shown as a loading control. 
Augustynek et al.

Gating mechanisms of Orai calcium channels

A

hO1
hO1-ANSGA
hO1-I249V-V252A-V255L-ANSGA
hO1-I249V-ANSGA
hO1-V252A-ANSGA
hO1-V255L-ANSGA
hO1-I249V-V255L-ANSGA

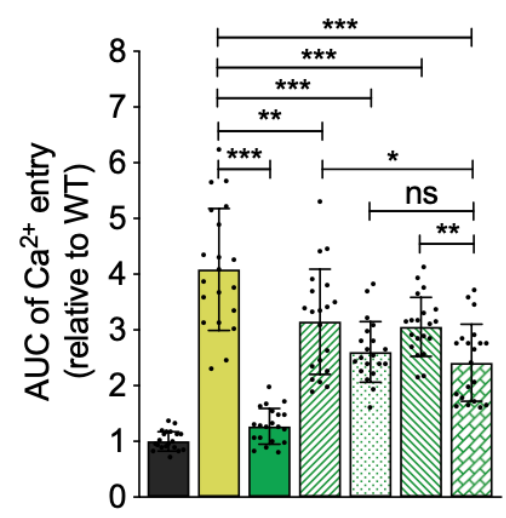

B
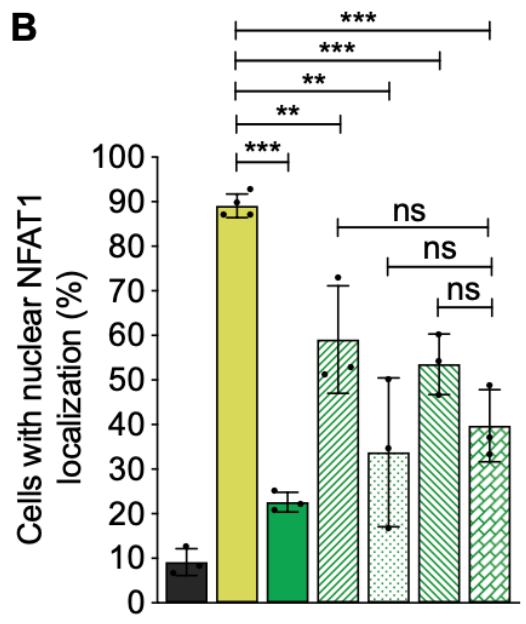

C

mCh-hO1-I249VV252A-V255LANSGA

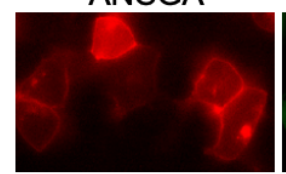

mCh-hO1-1249VANSGA

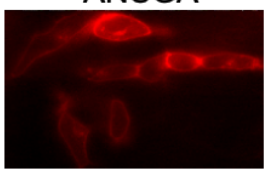

mCh-hO1-V252AANSGA

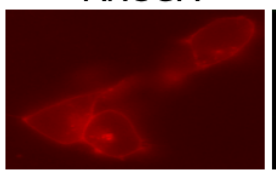

mCh-h01-V255LANSGA

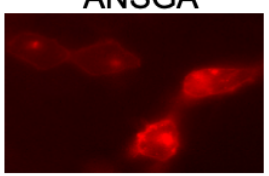

mCh-h01-I249VV255L-ANSGA

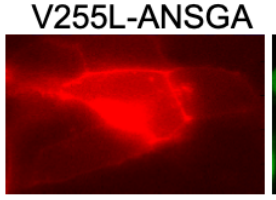

NFAT1-CFP

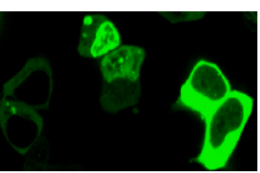

NFAT1-CFP

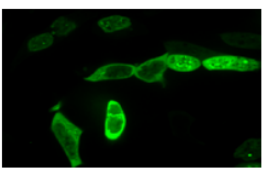

NFAT1-CFP

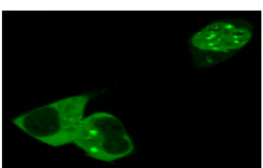

NFAT1-CFP

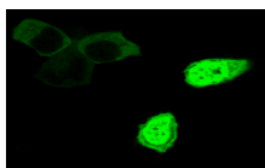

NFAT1-CFP

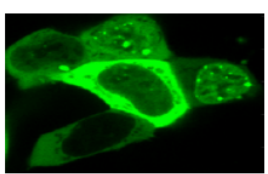

Overlay

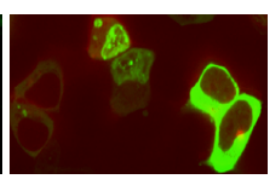

Overlay

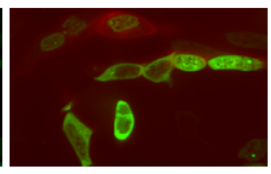

Overlay

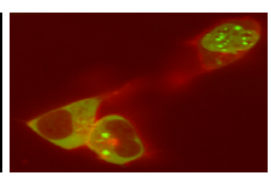

Overlay

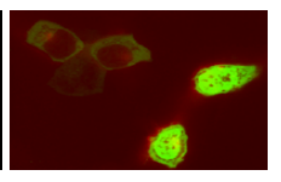

Overlay

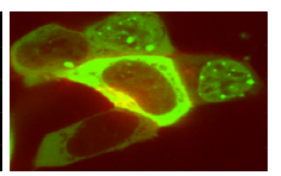

\section{Figure 7- figure supplement 2.}

883 (A) AUC quantifications of constitutive Ca2+ influx measured in HEK293 S1/S2 DKO cells expressing h01-ANSGA or other indicated 884 mutants of h01-ANSGA relative to WT h01 (mean \pm SD; n=20). (B) HEK293 cells expressing mCherry-h01, h01-ANSGA or I249V-V252AV255L, I249V, V252A, V255L and I249V-V255L mutants of h01-ANSGA with nuclear NFAT1-CFP localization shown as percentage (mean $\pm \mathrm{SD} ; \mathrm{n} \geq 3$ ). $p \leq 0.001$ is indicated as "***", $0.001<p \leq 0.01$ as "**”, $0.01<p<0.05$ as "*” and $p \geq 0.05$ as "ns". (C) Representative confocal microscopy images of HEK293 cells co-expressing NFAT1-CFP with either mCherry-h01, mCherry-h01-ANSGA or indicated mutants of mCherry-h01-ANSGA along with the CFP/mCherry overlay images. 
bioRxiv preprint doi: https://doi.org/10.1101/2021.10.26.465861; this version posted October 26, 2021. The copyright holder for this preprint (which was not certified by peer review) is the author/funder. All rights reserved. No reuse allowed without permission.

Augustynek et al.

Gating mechanisms of Orai calcium channels

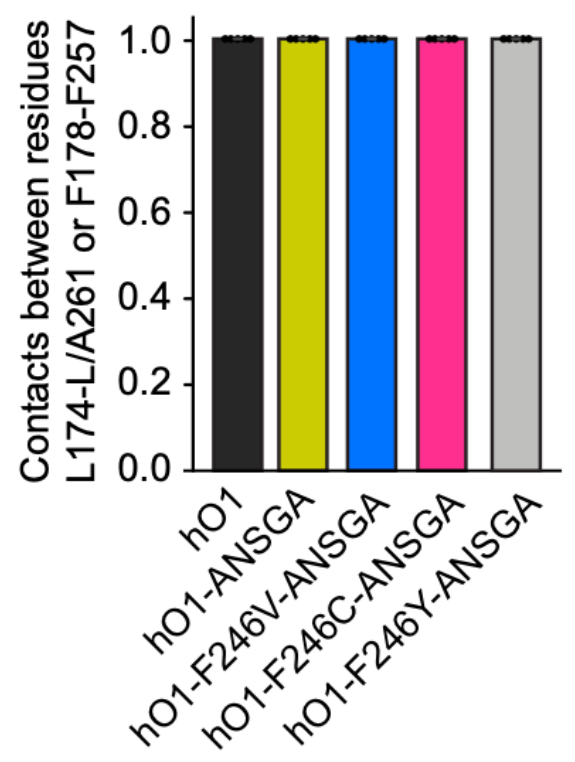

$890 \quad$ Figure 8- figure supplement 1.

891 Intra-subunit contact frequencies between residue pairs L174-L/A261 and F178-F257 averaged over each of the 5 MD trajectories are 892 shown for various simulation systems. Data are identical for both residue pairs. 
bioRxiv preprint doi: https://doi.org/10.1101/2021.10.26.465861; this version posted October 26, 2021. The copyright holder for this preprint (which was not certified by peer review) is the author/funder. All rights reserved. No reuse allowed without permission.

Augustynek et al.

Gating mechanisms of Orai calcium channels

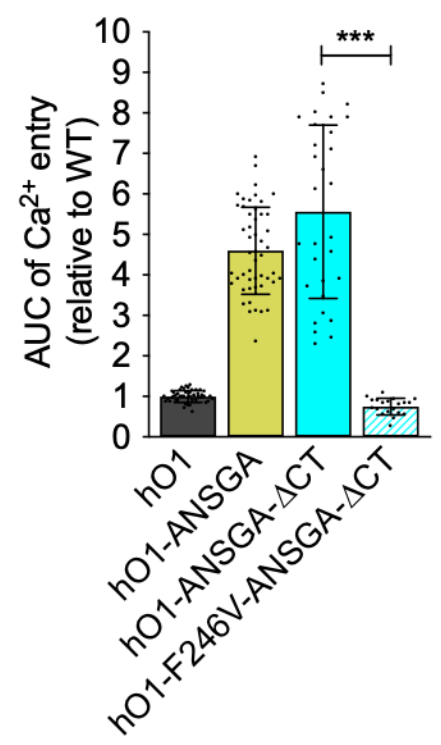

894 Figure 9- figure supplement 1. The TM4 extension beyond the ANSGA region of h01 is dispensable for F246V mediated inhibition 895 of constitutive activity.

896 The quantified AUC of constitutive $\mathrm{Ca}^{2+}$ entry recorded in HEK293 S1/S2 DKO cells expressing the indicated h01-ANSGA mutants relative

897 to the WT h01 channel (mean $\pm \mathrm{SD} ; \mathrm{n}=10$ ). $p \leq 0.001$ is indicated as "***". 
bioRxiv preprint doi: https://doi.org/10.1101/2021.10.26.465861; this version posted October 26, 2021. The copyright holder for this preprint (which was not certified by peer review) is the author/funder. All rights reserved. No reuse allowed without permission.

Augustynek et al.

Gating mechanisms of Orai calcium channels

\section{References}

Alansary, D., Bogeski, I., \& Niemeyer, B. A. (2015). Facilitation of Orai3 targeting and store-operated function by Orai1. Biochim Biophys Acta, 1853(7), 1541-1550. doi:10.1016/j.bbamcr.2015.03.007

Bakowski, D., Murray, F., \& Parekh, A. B. (2021). Store-Operated Ca(2+) Channels: Mechanism, Function, Pharmacology, and Therapeutic Targets. Annu Rev Pharmacol Toxicol, 61, 629-654. doi:10.1146/annurev-pharmtox-031620-105135

Baraniak, J. H., Jr., Zhou, Y., Nwokonko, R. M., Jennette, M. R., Kazzaz, S. A., Stenson, J. M., . . . Gill, D. L. (2021). Orai channel C-terminal peptides are key modulators of STIM-Orai coupling and calcium signal generation. Cell Rep, 35(13), 109322. doi:10.1016/j.celrep.2021.109322

Berna-Erro, A., Jardin, I., Salido, G. M., \& Rosado, J. A. (2017). Role of STIM2 in cell function and physiopathology. J Physiol, 595(10), 3111-3128. doi:10.1113/JP273889

Bhardwaj, R., Augustynek, B. S., Ercan-Herbst, E., Kandasamy, P., Seedorf, M., Peinelt, C., \& Hediger, M. A. (2020). Ca(2+)/Calmodulin Binding to STIM1 Hydrophobic Residues Facilitates Slow Ca(2+)-Dependent Inactivation of the Orai1 Channel. Cell Physiol Biochem, 54(2), 252-270. doi:10.33594/000000218

Bhardwaj, R., Hediger, M. A., \& Demaurex, N. (2016). Redox modulation of STIM-ORAI signaling. Cell Calcium, 60(2), 142-152. doi:10.1016/j.ceca.2016.03.006

Blankenship, J. R., \& Heitman, J. (2005). Calcineurin is required for Candida albicans to survive calcium stress in serum. Infect Immun, 73(9), 5767-5774. doi:10.1128/IAI.73.9.5767-5774.2005

Bonhenry, D., Schober, R., \& Schindl, R. (2021). Twisting gating residues in the Orai pore. Cell Calcium, 93, 102323. doi:10.1016/j.ceca.2020.102323

Brooks, B. R., Brooks, C. L., 3rd, Mackerell, A. D., Jr., Nilsson, L., Petrella, R. J., Roux, B., . . Karplus, M. (2009). CHARMM: the biomolecular simulation program. J Comput Chem, 30(10), 1545-1614. doi:10.1002/jcc.21287

Buchan, D. W., Minneci, F., Nugent, T. C., Bryson, K., \& Jones, D. T. (2013). Scalable web services for the PSIPRED Protein Analysis Workbench. Nucleic Acids Res, 41(Web Server issue), W349-357. doi:10.1093/nar/gkt381

Bulla, M., Gyimesi, G., Kim, J. H., Bhardwaj, R., Hediger, M. A., Frieden, M., \& Demaurex, N. (2019). ORAl1 channel gating and selectivity is differentially altered by natural mutations in the first or third transmembrane domain. J Physiol, 597(2), 561-582. doi:10.1113/JP277079

Butorac, C., Muik, M., Derler, I., Stadlbauer, M., Lunz, V., Krizova, A., . . Romanin, C. (2019). A novel STIM1Orai1 gating interface essential for CRAC channel activation. Cell Calcium, 79, 57-67. doi:10.1016/j.ceca.2019.02.009

Case, D. A., Ben-Shalom, I. Y., Brozell, S. R., Cerutti, D. S., Cheatham, I., T. E., Cruzeiro, V. W. D., . . Kollman, P. A. (2018). AMBER 2018. University of California, San Francisco.

Darbellay, B., Arnaudeau, S., Bader, C. R., Konig, S., \& Bernheim, L. (2011). STIM1L is a new actin-binding splice variant involved in fast repetitive Ca2+ release. J Cell Biol, 194(2), 335-346. doi:10.1083/jcb.201012157

Derler, I., Butorac, C., Krizova, A., Stadlbauer, M., Muik, M., Fahrner, M., . . Romanin, C. (2018). Authentic CRAC channel activity requires STIM1 and the conserved portion of the Orai N terminus. J Biol Chem, 293(4), 1259-1270. doi:10.1074/jbc.M117.812206

Derler, I., Plenk, P., Fahrner, M., Muik, M., Jardin, I., Schindl, R., . . . Romanin, C. (2013). The extended transmembrane Orai1 $\mathrm{N}$-terminal (ETON) region combines binding interface and gate for Orai1 activation by STIM1. J Biol Chem, 288(40), 29025-29034. doi:10.1074/jbc.M113.501510

Dong, H., Zhang, Y., Song, R., Xu, J., Yuan, Y., Liu, J., . . Klein, M. L. (2019). Toward a Model for Activation of Orai Channel. iScience, 16, 356-367. doi:10.1016/j.isci.2019.05.041

Ercan, E., Chung, S. H., Bhardwaj, R., \& Seedorf, M. (2012). Di-arginine signals and the K-rich domain retain the $\mathrm{Ca}(2)(+)$ sensor STIM1 in the endoplasmic reticulum. Traffic, 13(7), 992-1003. doi:10.1111/j.16000854.2012.01359.x

Fahrner, M., Pandey, S. K., Muik, M., Traxler, L., Butorac, C., Stadlbauer, M., . . Derler, I. (2018). Communication between $N$ terminus and loop2 tunes Orai activation. J Biol Chem, 293(4), 1271-1285. doi:10.1074/jbc.M117.812693

Feske, S., Gwack, Y., Prakriya, M., Srikanth, S., Puppel, S. H., Tanasa, B., . . Rao, A. (2006). A mutation in Orai1 causes immune deficiency by abrogating CRAC channel function. Nature, 441(7090), 179-185. doi:10.1038/nature04702

Fiser, A., Do, R. K., \& Sali, A. (2000). Modeling of loops in protein structures. Protein Sci, 9(9), 1753-1773. doi:10.1110/ps.9.9.1753 
bioRxiv preprint doi: https://doi.org/10.1101/2021.10.26.465861; this version posted October 26, 2021. The copyright holder for this preprint (which was not certified by peer review) is the author/funder. All rights reserved. No reuse allowed without permission.

Augustynek et al.

Gating mechanisms of Orai calcium channels

Frischauf, I., Litvinukova, M., Schober, R., Zayats, V., Svobodova, B., Bonhenry, D., . . Schindl, R. (2017). Transmembrane helix connectivity in Orai1 controls two gates for calcium-dependent transcription. Sci Signal, 10(507). doi:10.1126/scisignal.aao0358

Frischauf, I., Muik, M., Derler, I., Bergsmann, J., Fahrner, M., Schindl, R., . . Romanin, C. (2009). Molecular determinants of the coupling between STIM1 and Orai channels: differential activation of Orai1-3 channels by a STIM1 coiled-coil mutant. J Biol Chem, 284(32), 21696-21706. doi:10.1074/jbc.M109.018408

Frischauf, I., Schindl, R., Bergsmann, J., Derler, I., Fahrner, M., Muik, M., . . Romanin, C. (2011). Cooperativeness of Orai cytosolic domains tunes subtype-specific gating. J Biol Chem, 286(10), 8577-8584. doi:10.1074/jbc.M110.187179

Fukushima, M., Tomita, T., Janoshazi, A., \& Putney, J. W. (2012). Alternative translation initiation gives rise to two isoforms of Orai1 with distinct plasma membrane mobilities. J Cell Sci, 125(Pt 18), 4354-4361. doi:10.1242/jcs.104919

Gowers, R. J., Linke, M., Barnoud, J., Reddy, T. J. E., Melo, M. N., Seyler, S. L., . . Beckstein, O. (2016). MDAnalysis: A Python package for the rapid analysis of molecular dynamics simulations. . In S. Benthall and S. Rostrup, editors, Proceedings of the 15th Python in Science Conference, 98-105. doi:10.25080/Majora-629e541a-00e

Graham, S. J., Dziadek, M. A., \& Johnstone, L. S. (2011). A cytosolic STIM2 preprotein created by signal peptide inefficiency activates ORAI1 in a store-independent manner. J Biol Chem, 286(18), 16174-16185. doi:10.1074/jbc.M110.206946

Harmsen, T., Klaasen, S., van de Vrugt, H., \& Te Riele, H. (2018). DNA mismatch repair and oligonucleotide endprotection promote base-pair substitution distal from a CRISPR/Cas9-induced DNA break. Nucleic Acids Res, 46(6), 2945-2955. doi:10.1093/nar/gky076

He, L., Wang, L., Zeng, H., Tan, P., Ma, G., Zheng, S., . . Zhou, Y. (2021). Engineering of a bona fide lightoperated calcium channel. Nat Commun, 12(1), 164. doi:10.1038/s41467-020-20425-4

Hou, X., Burstein, S. R., \& Long, S. B. (2018). Structures reveal opening of the store-operated calcium channel Orai. Elife, 7. doi:10.7554/eLife.36758

Hou, X., Outhwaite, I. R., Pedi, L., \& Long, S. B. (2020). Cryo-EM structure of the calcium release-activated calcium channel Orai in an open conformation. Elife, 9. doi:10.7554/eLife.62772

Hou, X., Pedi, L., Diver, M. M., \& Long, S. B. (2012). Crystal structure of the calcium release-activated calcium channel Orai. Science, 338(6112), 1308-1313. doi:10.1126/science.1228757

Huang, J., Rauscher, S., Nawrocki, G., Ran, T., Feig, M., de Groot, B. L., . . MacKerell, A. D., Jr. (2017). CHARMM36m: an improved force field for folded and intrinsically disordered proteins. Nat Methods, 14(1), 71-73. doi:10.1038/nmeth.4067

Jo, S., Cheng, X., Islam, S. M., Huang, L., Rui, H., Zhu, A., . . Im, W. (2014). CHARMM-GUI PDB manipulator for advanced modeling and simulations of proteins containing nonstandard residues. Adv Protein Chem Struct Biol, 96, 235-265. doi:10.1016/bs.apcsb.2014.06.002

Jo, S., Kim, T., \& Im, W. (2007). Automated builder and database of protein/membrane complexes for molecular dynamics simulations. PLoS One, 2(9), e880. doi:10.1371/journal.pone.0000880

Jo, S., Kim, T., Iyer, V. G., \& Im, W. (2008). CHARMM-GUI: a web-based graphical user interface for CHARMM. J Comput Chem, 29(11), 1859-1865. doi:10.1002/jcc.20945

Jo, S., Lim, J. B., Klauda, J. B., \& Im, W. (2009). CHARMM-GUI Membrane Builder for mixed bilayers and its application to yeast membranes. Biophys J, 97(1), 50-58. doi:10.1016/j.bpj.2009.04.013

Jones, D. T. (1999). Protein secondary structure prediction based on position-specific scoring matrices. J Mol Biol, 292(2), 195-202. doi:10.1006/jmbi.1999.3091

Kar, P., Lin, Y. P., Bhardwaj, R., Tucker, C. J., Bird, G. S., Hediger, M. A., . . Parekh, A. B. (2021). The N terminus of Orai1 couples to the AKAP79 signaling complex to drive NFAT1 activation by local $\mathrm{Ca}(2+)$ entry. Proc Natl Acad Sci U S A, 118(19). doi:10.1073/pnas.2012908118

Kim, K. M., Wijerathne, T., Hur, J. H., Kang, U. J., Kim, I. H., Kweon, Y. C., . . Park, C. Y. (2018). Distinct gating mechanism of SOC channel involving STIM-Orai coupling and an intramolecular interaction of Orai in Caenorhabditis elegans. Proc Natl Acad Sci U S A, 115(20), E4623-E4632. doi:10.1073/pnas.1714986115

Knapp, M. L., Förderer, K., Alansary, D., Jung, M., Schwarz, Y., Lis, A., \& Niemeyer, B. (2020). Alternative splicing switches STIM1 targeting to specialized membrane contact sites and modifies SOCE. bioRxiv. doi:https://doi.org/10.1101/2020.03.25.005199

Krizova, A., Maltan, L., \& Derler, I. (2019). Critical parameters maintaining authentic CRAC channel hallmarks. Eur Biophys J, 48(5), 425-445. doi:10.1007/s00249-019-01355-6 
bioRxiv preprint doi: https://doi.org/10.1101/2021.10.26.465861; this version posted October 26, 2021. The copyright holder for this preprint (which was not certified by peer review) is the author/funder. All rights reserved. No reuse allowed without permission.

Augustynek et al.

Gating mechanisms of Orai calcium channels

Larkin, M. A., Blackshields, G., Brown, N. P., Chenna, R., McGettigan, P. A., McWilliam, H., . . Higgins, D. G. (2007). Clustal $W$ and Clustal $X$ version 2.0. Bioinformatics, 23(21), 2947-2948. doi:10.1093/bioinformatics/btm404

Lee, J., Cheng, X., Swails, J. M., Yeom, M. S., Eastman, P. K., Lemkul, J. A., . . Im, W. (2016). CHARMM-GUI Input Generator for NAMD, GROMACS, AMBER, OpenMM, and CHARMM/OpenMM Simulations Using the CHARMM36 Additive Force Field. $J$ Chem Theory Comput, 12(1), 405-413. doi:10.1021/acs.jctc.5b00935

Li, S., Yao, M., Niu, C., Liu, D., Tang, Z., Gu, C., . . . Wu, F. (2019). Inhibition of MCF-7 breast cancer cell proliferation by a synthetic peptide derived from the C-terminal sequence of Orai channel. Biochem Biophys Res Commun, 516(4), 1066-1072. doi:10.1016/j.bbrc.2019.06.153

Li, Z., Liu, L., Deng, Y., Ji, W., Du, W., Xu, P., . . Xu, T. (2011). Graded activation of CRAC channel by binding of different numbers of STIM1 to Orai1 subunits. Cell Res, 21(2), 305-315. doi:10.1038/cr.2010.131

Li, Z., Lu, J., Xu, P., Xie, X., Chen, L., \& Xu, T. (2007). Mapping the interacting domains of STIM1 and Orai1 in $\mathrm{Ca} 2+$ release-activated Ca2+ channel activation. J Biol Chem, 282(40), 29448-29456. doi:10.1074/jbc.M703573200

Liou, J., Kim, M. L., Heo, W. D., Jones, J. T., Myers, J. W., Ferrell, J. E., Jr., \& Meyer, T. (2005). STIM is a Ca2+ sensor essential for Ca2+-store-depletion-triggered Ca2+ influx. Curr Biol, 15(13), 1235-1241. doi:10.1016/j.cub.2005.05.055

Lis, A., Peinelt, C., Beck, A., Parvez, S., Monteilh-Zoller, M., Fleig, A., \& Penner, R. (2007). CRACM1, CRACM2, and CRACM3 are store-operated Ca2+ channels with distinct functional properties. Curr Biol, 17(9), 794800. doi:10.1016/j.cub.2007.03.065

Lis, A., Zierler, S., Peinelt, C., Fleig, A., \& Penner, R. (2010). A single lysine in the N-terminal region of storeoperated channels is critical for STIM1-mediated gating. J Gen Physiol, 136(6), 673-686. doi:10.1085/jgp.201010484

Liu, X., Wu, G., Yu, Y., Chen, X., Ji, R., Lu, J., . . Shen, Y. (2019). Molecular understanding of calcium permeation through the open Orai channel. PLoS Biol, 17(4), e3000096. doi:10.1371/journal.pbio.3000096

Lomize, M. A., Lomize, A. L., Pogozheva, I. D., \& Mosberg, H. I. (2006). OPM: orientations of proteins in membranes database. Bioinformatics, 22(5), 623-625. doi:10.1093/bioinformatics/btk023

McNally, B. A., Somasundaram, A., Jairaman, A., Yamashita, M., \& Prakriya, M. (2013). The C- and N-terminal STIM1 binding sites on Orai1 are required for both trapping and gating CRAC channels. J Physiol, 591(11), 2833-2850. doi:10.1113/jphysiol.2012.250456

McNally, B. A., Somasundaram, A., Yamashita, M., \& Prakriya, M. (2012). Gated regulation of CRAC channel ion selectivity by STIM1. Nature, 482(7384), 241-245. doi:10.1038/nature10752

Mercer, J. C., Dehaven, W. I., Smyth, J. T., Wedel, B., Boyles, R. R., Bird, G. S., \& Putney, J. W., Jr. (2006). Large store-operated calcium selective currents due to co-expression of Orai1 or Orai2 with the intracellular calcium sensor, Stim1. J Biol Chem, 281(34), 24979-24990. doi:10.1074/jbc.M604589200

Michaud-Agrawal, N., Denning, E. J., Woolf, T. B., \& Beckstein, O. (2011). MDAnalysis: a toolkit for the analysis of molecular dynamics simulations. J Comput Chem, 32(10), 2319-2327. doi:10.1002/jcc.21787

Miederer, A. M., Alansary, D., Schwar, G., Lee, P. H., Jung, M., Helms, V., \& Niemeyer, B. A. (2015). A STIM2 splice variant negatively regulates store-operated calcium entry. Nat Commun, 6, 6899. doi:10.1038/ncomms7899

Muik, M., Frischauf, I., Derler, I., Fahrner, M., Bergsmann, J., Eder, P., . . Romanin, C. (2008). Dynamic coupling of the putative coiled-coil domain of ORAl1 with STIM1 mediates ORAI1 channel activation. $J$ Biol Chem, 283(12), 8014-8022. doi:10.1074/jbc.M708898200

Navarro-Borelly, L., Somasundaram, A., Yamashita, M., Ren, D., Miller, R. J., \& Prakriya, M. (2008). STIM1-Orai1 interactions and Orai1 conformational changes revealed by live-cell FRET microscopy. J Physiol, 586(22), 5383-5401. doi:10.1113/jphysiol.2008.162503

Nesin, V., Wiley, G., Kousi, M., Ong, E. C., Lehmann, T., Nicholl, D. J., . . Tsiokas, L. (2014). Activating mutations in STIM1 and ORAl1 cause overlapping syndromes of tubular myopathy and congenital miosis. Proc Natl Acad Sci U S A, 111(11), 4197-4202. doi:10.1073/pnas.1312520111

Niemeyer, B. A. (2016). Changing calcium: CRAC channel (STIM and Orai) expression, splicing, and posttranslational modifiers. Am J Physiol Cell Physiol, 310(9), C701-709. doi:10.1152/ajpcell.00034.2016

Niu, L., Wu, F., Li, K., Li, J., Zhang, S. L., Hu, J., \& Wang, Q. (2020). STIM1 interacts with termini of Orai channels in a sequential manner. J Cell Sci, 133(8). doi:10.1242/jcs.239491

Palty, R., Stanley, C., \& Isacoff, E. Y. (2015). Critical role for Orai1 C-terminal domain and TM4 in CRAC channel gating. Cell Res, 25(8), 963-980. doi:10.1038/cr.2015.80 
bioRxiv preprint doi: https://doi.org/10.1101/2021.10.26.465861; this version posted October 26, 2021. The copyright holder for this preprint (which was not certified by peer review) is the author/funder. All rights reserved. No reuse allowed without permission.

Augustynek et al.

Gating mechanisms of Orai calcium channels

Park, C. Y., Hoover, P. J., Mullins, F. M., Bachhawat, P., Covington, E. D., Raunser, S., . . Lewis, R. S. (2009). STIM1 clusters and activates CRAC channels via direct binding of a cytosolic domain to Orai1. Cell, 136(5), 876-890. doi:10.1016/j.cell.2009.02.014

Prakriya, M., Feske, S., Gwack, Y., Srikanth, S., Rao, A., \& Hogan, P. G. (2006). Orai1 is an essential pore subunit of the CRAC channel. Nature, 443(7108), 230-233. doi:10.1038/nature05122

Ramesh, G., Jarzembowski, L., Schwarz, Y., Poth, V., Konrad, M., Knapp, M. L., . . Niemeyer, B. A. (2021). A short isoform of STIM1 confers frequency-dependent synaptic enhancement. Cell Rep, 34(11), 108844. doi:10.1016/j.celrep.2021.108844

Rana, A., Yen, M., Sadaghiani, A. M., Malmersjo, S., Park, C. Y., Dolmetsch, R. E., \& Lewis, R. S. (2015). Alternative splicing converts STIM2 from an activator to an inhibitor of store-operated calcium channels. $J$ Cell Biol, 209(5), 653-669. doi:10.1083/jcb.201412060

Roos, J., DiGregorio, P. J., Yeromin, A. V., Ohlsen, K., Lioudyno, M., Zhang, S., . . Stauderman, K. A. (2005). STIM1, an essential and conserved component of store-operated Ca2+ channel function. J Cell Biol, 169(3), 435-445. doi:10.1083/jcb.200502019

Sali, A., \& Blundell, T. L. (1993). Comparative protein modelling by satisfaction of spatial restraints. J Mol Biol, 234(3), 779-815. doi:10.1006/jmbi.1993.1626

Schild, A., Bhardwaj, R., Wenger, N., Tscherrig, D., Kandasamy, P., Dernic, J., . . Lochner, M. (2020). Synthesis and Pharmacological Characterization of 2-Aminoethyl Diphenylborinate (2-APB) Derivatives for Inhibition of Store-Operated Calcium Entry (SOCE) in MDA-MB-231 Breast Cancer Cells. Int J Mol Sci, 21(16). doi:10.3390/ijms21165604

Schober, R., Bonhenry, D., Lunz, V., Zhu, J., Krizova, A., Frischauf, I., . . Schindl, R. (2019). Sequential activation of STIM1 links $\mathrm{Ca}(2+)$ with luminal domain unfolding. Sci Signal, 12(608). doi:10.1126/scisignal.aax3194

Simonin, A., \& Fuster, D. (2010). Nedd4-1 and beta-arrestin-1 are key regulators of $\mathrm{Na}+/ \mathrm{H}+$ exchanger 1 ubiquitylation, endocytosis, and function. J Biol Chem, 285(49), 38293-38303. doi:10.1074/jbc.M110.115089

Smart, O. S., Goodfellow, J. M., \& Wallace, B. A. (1993). The pore dimensions of gramicidin A. Biophys J, 65(6), 2455-2460. doi:10.1016/S0006-3495(93)81293-1

Smart, O. S., Neduvelii, J. G., Wang, X., Wallace, B. A., \& Sansom, M. S. (1996). HOLE: a program for the analysis of the pore dimensions of ion channel structural models. J Mol Graph, 14(6), 354-360, 376. doi:10.1016/s0263-7855(97)00009-x

Tiffner, A., Maltan, L., Fahrner, M., Sallinger, M., Weiss, S., Grabmayr, H., . . . Derler, I. (2021). Transmembrane Domain 3 (TM3) Governs Orai1 and Orai3 Pore Opening in an Isoform-Specific Manner. Front Cell Dev Biol, 9, 635705. doi:10.3389/fcell.2021.635705

Tiffner, A., Schober, R., Hoeglinger, C., Bonhenry, D., Pandey, S., Lunz, V., . . Derler, I. (2020). CRAC channel opening is determined by a series of Orai1 gating checkpoints in the transmembrane and cytosolic regions. J Biol Chem. doi:10.1074/jbc.RA120.015548

Tirado-Lee, L., Yamashita, M., \& Prakriya, M. (2015). Conformational Changes in the Orai1 C-Terminus Evoked by STIM1 Binding. PLoS One, 10(6), e0128622. doi:10.1371/journal.pone.0128622

Vig, M., Peinelt, C., Beck, A., Koomoa, D. L., Rabah, D., Koblan-Huberson, M., . . Kinet, J. P. (2006). CRACM1 is a plasma membrane protein essential for store-operated Ca2+ entry. Science, 312(5777), 1220-1223. doi:10.1126/science.1127883

Webb, B., \& Sali, A. (2016). Comparative Protein Structure Modeling Using MODELLER. Curr Protoc Bioinformatics, 54, 56 1-5 6 37. doi:10.1002/cpbi.3

Wu, E. L., Cheng, X., Jo, S., Rui, H., Song, K. C., Davila-Contreras, E. M., . . Im, W. (2014). CHARMM-GUI Membrane Builder toward realistic biological membrane simulations. J Comput Chem, 35(27), 1997-2004. doi:10.1002/jcc.23702

Yamashita, M., Yeung, P. S., Ing, C. E., McNally, B. A., Pomes, R., \& Prakriya, M. (2017). STIM1 activates CRAC channels through rotation of the pore helix to open a hydrophobic gate. Nat Commun, 8, 14512. doi:10.1038/ncomms14512

Yeung, P. S., Ing, C. E., Yamashita, M., Pomes, R., \& Prakriya, M. (2020). A sulfur-aromatic gate latch is essential for opening of the Orai1 channel pore. Elife, 9. doi:10.7554/eLife.60751

Yeung, P. S., Yamashita, M., Ing, C. E., Pomes, R., Freymann, D. M., \& Prakriya, M. (2018). Mapping the functional anatomy of Orai1 transmembrane domains for CRAC channel gating. Proc Natl Acad Sci U S A, 115(22), E5193-E5202. doi:10.1073/pnas.1718373115

Yeung, P. S., Yamashita, M., \& Prakriya, M. (2020). Molecular basis of allosteric Orai1 channel activation by STIM1. J Physiol, 598(9), 1707-1723. doi:10.1113/JP276550

Zheng, H., Zhou, M. H., Hu, C., Kuo, E., Peng, X., Hu, J., . . . Zhang, S. L. (2013). Differential roles of the C and $\mathrm{N}$ termini of Orai1 protein in interacting with stromal interaction molecule 1 (STIM1) for Ca2+ release- 
bioRxiv preprint doi: https://doi.org/10.1101/2021.10.26.465861; this version posted October 26, 2021. The copyright holder for this preprint (which was not certified by peer review) is the author/funder. All rights reserved. No reuse allowed without permission.

Augustynek et al.

Gating mechanisms of Orai calcium channels

activated Ca2+ (CRAC) channel activation. J Biol Chem, 288(16), 11263-11272. doi:10.1074/jbc.M113.450254

Zhou, L., Chi, X., Zhu, Y., Zhang, T., Liu, J., Ma, G., . . Wang, Y. (2018). Digitoxin Suppresses Store Operated Calcium Entry by Modulating Phosphorylation and the Pore Region of Orai1. Curr Mol Med, 18(6), 392399. doi:10.2174/1566524018666181113111316

Zhou, Y., Cai, X., Loktionova, N. A., Wang, X., Nwokonko, R. M., Wang, X., . . Gill, D. L. (2016). The STIM1binding site nexus remotely controls Orai1 channel gating. Nat Commun, 7, 13725. doi:10.1038/ncomms 13725

Zhou, Y., Nwokonko, R. M., Baraniak, J. H., Jr., Trebak, M., Lee, K. P. K., \& Gill, D. L. (2019). The remote allosteric control of Orai channel gating. PLoS Biol, 17(8), e3000413. doi:10.1371/journal.pbio.3000413 
bioRxiv preprint doi: https://doi.org/10.1101/2021.10.26.465861; this version posted October 26, 2021. The copyright holder for this preprint (which was not certified by peer review) is the author/funder. All rights reserved. No reuse allowed without permission.

Augustynek et al.

Gating mechanisms of Orai calcium channels

\section{Key resources table}

\begin{tabular}{|c|c|c|c|}
\hline $\begin{array}{l}\text { Reagent type } \\
\text { (species) or } \\
\text { resource }\end{array}$ & Designation & $\begin{array}{l}\text { Source or } \\
\text { reference }\end{array}$ & Additional information \\
\hline $\begin{array}{l}\text { Gene Drosophila } \\
\text { melanogaster }\end{array}$ & ORAI & $\mathrm{NCBI}$ & $\begin{array}{l}\text { Gene ID: } \\
37040\end{array}$ \\
\hline $\begin{array}{l}\text { Gene Xenopus } \\
\text { laevis }\end{array}$ & orai1 & $\mathrm{NCBI}$ & $\begin{array}{l}\text { Gene ID: } \\
403390\end{array}$ \\
\hline Gene (Mouse) & Orai1 & $\mathrm{NCBI}$ & $\begin{array}{l}\text { Gene ID: } \\
109305\end{array}$ \\
\hline Gene (Human) & ORAl1 & $\mathrm{NCBI}$ & $\begin{array}{l}\text { Gene ID: } \\
84876\end{array}$ \\
\hline Gene (Human) & ORAI2 & $\mathrm{NCBI}$ & $\begin{array}{l}\text { Gene ID: } \\
80228\end{array}$ \\
\hline Gene (Human) & ORAI3 & $\mathrm{NCBI}$ & $\begin{array}{l}\text { Gene ID: } \\
93129\end{array}$ \\
\hline Gene (Human) & STIM1 & $\mathrm{NCBI}$ & $\begin{array}{l}\text { Gene ID: } \\
6786\end{array}$ \\
\hline $\begin{array}{l}\text { Cell line } \\
\text { (Homo sapiens) }\end{array}$ & HEK-293 & ATCC & CRL-1573 \\
\hline $\begin{array}{l}\text { Cell line } \\
\text { (Homo sapiens) }\end{array}$ & HEK-293T & ATCC & CRL-3216 \\
\hline $\begin{array}{l}\text { Chemical } \\
\text { compound, drug }\end{array}$ & Opti-MEM & $\begin{array}{l}\text { Thermo Fisher } \\
\text { Scientific }\end{array}$ & $\begin{array}{l}\text { Cat\# } \\
11058021 \\
\end{array}$ \\
\hline $\begin{array}{l}\text { Chemical } \\
\text { compound, drug }\end{array}$ & $\begin{array}{l}\text { Lipofectamine } 2000 \\
\text { Transfection Reagent }\end{array}$ & $\begin{array}{l}\text { Thermo Fisher } \\
\text { Scientific }\end{array}$ & $\begin{array}{l}\text { Cat\# } \\
11668019\end{array}$ \\
\hline $\begin{array}{l}\text { Chemical } \\
\text { compound, drug }\end{array}$ & $\begin{array}{l}\text { Dulbecco's Modified } \\
\text { Eagle's Medium - high } \\
\text { glucose }\end{array}$ & $\begin{array}{l}\text { Thermo Fisher } \\
\text { Scientific }\end{array}$ & $\begin{array}{l}\text { Cat\# } \\
41965-039\end{array}$ \\
\hline $\begin{array}{l}\text { Chemical } \\
\text { compound, drug }\end{array}$ & $\begin{array}{l}\text { Dulbecco's Modified } \\
\text { Eagle's Medium - high } \\
\text { glucose, no calcium }\end{array}$ & $\begin{array}{l}\text { Thermo Fisher } \\
\text { Scientific }\end{array}$ & $\begin{array}{l}\text { Cat\# } \\
21068028\end{array}$ \\
\hline $\begin{array}{l}\text { Chemical } \\
\text { compound, drug }\end{array}$ & $\begin{array}{l}\text { Fetal Bovine Serum } \\
\text { (FBS) }\end{array}$ & Merck & $\begin{array}{l}\text { Cat\# } \\
\text { F7524 }\end{array}$ \\
\hline $\begin{array}{l}\text { Chemical } \\
\text { compound, drug }\end{array}$ & $\begin{array}{l}\text { MEM Non-essential } \\
\text { Amino Acid Solution } \\
(100 \mathrm{X})\end{array}$ & Merck & $\begin{array}{l}\text { Cat\# } \\
\text { M7145 }\end{array}$ \\
\hline $\begin{array}{l}\text { Chemical } \\
\text { compound, drug }\end{array}$ & $\begin{array}{l}\text { Poly-D-lysine } \\
\text { hydrobromide }\end{array}$ & Merck & $\begin{array}{l}\text { Cat\# } \\
\text { P6407 }\end{array}$ \\
\hline $\begin{array}{l}\text { Chemical } \\
\text { compound, drug }\end{array}$ & Penicillin-Streptomycin & Merck & $\begin{array}{l}\text { Cat\# } \\
\text { P4333 }\end{array}$ \\
\hline $\begin{array}{l}\text { Chemical } \\
\text { compound, drug }\end{array}$ & HEPES solution & Merck & $\begin{array}{l}\text { Cat\# } \\
\text { H0887 }\end{array}$ \\
\hline $\begin{array}{l}\text { Chemical } \\
\text { compound, drug }\end{array}$ & Ampicillin & AppliChem & $\begin{array}{l}\text { Cat\# } \\
\text { A0839 }\end{array}$ \\
\hline $\begin{array}{l}\text { Chemical } \\
\text { compound, drug }\end{array}$ & $\begin{array}{l}\text { D-myo-inositol 1, } \\
\text { 4,5-trisphosphate, } \\
\text { trisodium salt }\left(\mathrm{IP}_{3}\right)\end{array}$ & Calbiochem & $\begin{array}{l}\text { Cat\# } \\
407137\end{array}$ \\
\hline $\begin{array}{l}\text { Chemical } \\
\text { compound, drug }\end{array}$ & $\begin{array}{l}\text { Pfu Ultra High-fidelity } \\
\text { DNA Polymerase }\end{array}$ & $\begin{array}{l}\text { Agilent } \\
\text { Technologies }\end{array}$ & $\begin{array}{l}\text { Cat\# } \\
600380\end{array}$ \\
\hline $\begin{array}{l}\text { Chemical } \\
\text { compound, drug }\end{array}$ & $\begin{array}{l}\text { Bbsl-HF }{ }^{\circledR} \text { restriction } \\
\text { enzyme }\end{array}$ & $\begin{array}{l}\text { New England } \\
\text { BioLabs Inc. }\end{array}$ & $\begin{array}{l}\text { Cat\# } \\
\text { R3539s }\end{array}$ \\
\hline $\begin{array}{l}\text { Chemical } \\
\text { compound, drug }\end{array}$ & T4 DNA Ligase & $\begin{array}{l}\text { New England } \\
\text { BioLabs Inc. }\end{array}$ & $\begin{array}{l}\text { Cat\# } \\
\text { M0202S }\end{array}$ \\
\hline $\begin{array}{l}\text { Commercial assay } \\
\text { or kit }\end{array}$ & FLIPR Calcium 5 & $\begin{array}{l}\text { Molecular } \\
\text { Devices }\end{array}$ & $\begin{array}{l}\text { Cat\# } \\
\text { R8186 }\end{array}$ \\
\hline $\begin{array}{l}\text { Commercial assay } \\
\text { or kit }\end{array}$ & $\begin{array}{l}\text { Corning } 96 \text { Well Black } \\
\text { Polystyrene Microplate }\end{array}$ & Merck & $\begin{array}{l}\text { Cat\# } \\
\text { CLS3603 }\end{array}$ \\
\hline $\begin{array}{l}\text { Recombinant } \\
\text { DNA reagent }\end{array}$ & $\begin{array}{l}\text { pU6-(Bbsl)_CBh- } \\
\text { Cas9-T2A-mCherry } \\
\text { CRISPR plasmid }\end{array}$ & Addgene & $\begin{array}{l}\text { Plasmid } \\
\# 64324\end{array}$ \\
\hline
\end{tabular}


bioRxiv preprint doi: https://doi.org/10.1101/2021.10.26.465861; this version posted October 26, 2021. The copyright holder for this preprint (which was not certified by peer review) is the author/funder. All rights reserved. No reuse allowed without permission.

Augustynek et al.

Gating mechanisms of Orai calcium channels

\begin{tabular}{|c|c|c|c|c|}
\hline $\begin{array}{l}\text { Recombinant } \\
\text { DNA reagent }\end{array}$ & $\begin{array}{l}\text { pX330.pgkpuro } \\
\text { CRISPR plasmid }\end{array}$ & $\begin{array}{l}\text { (Harmsen et al., } \\
\text { 2018) }\end{array}$ & & \\
\hline $\begin{array}{l}\text { Recombinant } \\
\text { DNA reagent }\end{array}$ & Xenopus Orai 1 cDNA & $\begin{array}{l}\text { Horizon } \\
\text { Discovery Ltd. }\end{array}$ & $\begin{array}{l}\text { Clone ID: } \\
\text { 4633914, Cat\# } \\
\text { MXL1736- } \\
202771770 \\
\end{array}$ & \\
\hline $\begin{array}{l}\text { Transfection } \\
\text { construct }\end{array}$ & $\begin{array}{l}\text { mCherry- Xenopus } \\
\text { WT Orai } 1 \text { in } \\
\text { pmCherry-C1 }\end{array}$ & This study & & \\
\hline $\begin{array}{l}\text { Transfection } \\
\text { construct }\end{array}$ & $\begin{array}{l}\text { mCherry- Human WT } \\
\text { Orai1 in pmCherry-C1 }\end{array}$ & $\begin{array}{l}\text { Dr. Matthias } \\
\text { Seedorf, } \\
\text { Heidelberg } \\
\text { University, } \\
\text { Germany }\end{array}$ & & \\
\hline $\begin{array}{l}\text { Transfection } \\
\text { construct }\end{array}$ & $\begin{array}{l}\text { mCherry- Human WT } \\
\text { Orai3 in pmCherry-C1 }\end{array}$ & $\begin{array}{l}\text { Dr. Matthias } \\
\text { Seedorf, } \\
\text { Heidelberg } \\
\text { University, } \\
\text { Germany }\end{array}$ & & \\
\hline $\begin{array}{l}\text { Transfection } \\
\text { construct }\end{array}$ & $\begin{array}{l}\text { mCherry- Human } \\
\text { Orai1 P245L } \\
\text { pmCherry-C1 }\end{array}$ & $\begin{array}{l}\text { (Bulla et al., } \\
\text { 2019) }\end{array}$ & & \\
\hline $\begin{array}{l}\text { Transfection } \\
\text { construct }\end{array}$ & $\begin{array}{l}\text { mCherry- Human WT } \\
\text { Orai2 in pmCherry-C1 }\end{array}$ & This study & & \\
\hline $\begin{array}{l}\text { Transfection } \\
\text { construct }\end{array}$ & $\begin{array}{l}\text { Mouse WT Orai1- } \\
\text { eGFP in peGFP-N1 }\end{array}$ & $\begin{array}{l}\text { Prof. Francisco } \\
\text { Javier Martin- } \\
\text { Romero, } \\
\text { University of } \\
\text { Extremadura, } \\
\text { Spain }\end{array}$ & & \\
\hline $\begin{array}{l}\text { Transfection } \\
\text { construct }\end{array}$ & $\begin{array}{l}\text { 8X-His-mCherry- } \\
\text { Drosophila WT Orai1 } \\
\text { in pNmCherry }\end{array}$ & $\begin{array}{l}\text { (Hou et al., } \\
2018)\end{array}$ & & \\
\hline $\begin{array}{l}\text { Transfection } \\
\text { construct }\end{array}$ & CFP-NFATc1 & $\begin{array}{l}\text { (Schober et al., } \\
\text { 2019) }\end{array}$ & & \\
\hline $\begin{array}{l}\text { Transfection } \\
\text { construct }\end{array}$ & $\begin{array}{l}\text { Human STIM1 gRNA1 } \\
\text { in pX330.pgkpuro } \\
\text { CRISPR plasmid }\end{array}$ & This study & & \\
\hline $\begin{array}{l}\text { Transfection } \\
\text { construct }\end{array}$ & $\begin{array}{l}\text { Human STIM1 gRNA2 } \\
\text { in pX330.pgkpuro } \\
\text { CRISPR plasmid } \\
\end{array}$ & This study & & \\
\hline $\begin{array}{l}\text { Transfection } \\
\text { construct }\end{array}$ & $\begin{array}{l}\text { Human STIM2 gRNA1 } \\
\text { in pX330.pgkpuro } \\
\text { CRISPR plasmid }\end{array}$ & This study & & \\
\hline $\begin{array}{l}\text { Transfection } \\
\text { construct }\end{array}$ & $\begin{array}{l}\text { Human STIM2 gRNA2 } \\
\text { in pU6-(Bbsl)_CBh- } \\
\text { Cas9-T2A-mCherry } \\
\text { CRISPR plasmid }\end{array}$ & This study & & \\
\hline $\begin{array}{l}\text { Strain, strain } \\
\text { background } \\
\text { (Escherichia coli) }\end{array}$ & $\mathrm{DH} 5 \mathrm{a}$ & Invitrogen & $\begin{array}{l}\text { Cat \# } \\
18265-017\end{array}$ & \\
\hline $\begin{array}{l}\text { Sequenced- } \\
\text { based reagent }\end{array}$ & $\begin{array}{l}\text { H134A primers for } \\
\text { mutagenesis of } \\
\text { Human Orai1 }\end{array}$ & Microsynth AG & This study & $\begin{array}{l}\text { Forward primer: } \\
\text { 5'-CAC AGT GCT GGT GGC TGT GgC } \\
\text { CCT GTT TGC GCT CAT GAT C-3' }\end{array}$ \\
\hline $\begin{array}{l}\text { Sequenced- } \\
\text { based reagent }\end{array}$ & $\begin{array}{l}\text { H108A primers for } \\
\text { mutagenesis of } \\
\text { Human Orai2 }\end{array}$ & Microsynth AG & This study & $\begin{array}{l}\text { Forward primer: } \\
\text { 5'-CAC GGT GCT GGT GGC CGT GgC } \\
\text { CCT GTT CGC CCT CCT CAT C-3' }\end{array}$ \\
\hline $\begin{array}{l}\text { Sequenced- } \\
\text { based reagent }\end{array}$ & $\begin{array}{l}\text { H109A primers for } \\
\text { mutagenesis of } \\
\text { Human Orai3 }\end{array}$ & Microsynth AG & This study & $\begin{array}{l}\text { Forward primer: } \\
\text { 5'-CAC CGT GCT GGT GGC TGT GgC } \\
\text { CCT CTT TGC ACT CAT GGT C-3' }\end{array}$ \\
\hline
\end{tabular}


bioRxiv preprint doi: https://doi.org/10.1101/2021.10.26.465861; this version posted October 26, 2021. The copyright holder for this preprint (which was not certified by peer review) is the author/funder. All rights reserved. No reuse allowed without permission.

Augustynek et al.

Gating mechanisms of Orai calcium channels

\begin{tabular}{|c|c|c|c|c|}
\hline $\begin{array}{l}\text { Sequenced- } \\
\text { based reagent }\end{array}$ & $\begin{array}{l}\text { L261A-V262N-H264G- } \\
\text { K265A (ANSGA) } \\
\text { primers for } \\
\text { mutagenesis of } \\
\text { Human Orai1 }\end{array}$ & Microsynth AG & This study & $\begin{array}{l}\text { Forward primer: } \\
\text { 5'-GTC CAC TTC TAC CGC TCA gcG } \\
\text { aaT AGC ggT, gcG ACC GAC CGA } \\
\text { CAG TTC C-3' }\end{array}$ \\
\hline $\begin{array}{l}\text { Sequenced- } \\
\text { based reagent }\end{array}$ & $\begin{array}{l}\text { L222A-V223N-H225G- } \\
\text { K226A (ANRGA) } \\
\text { primers for } \\
\text { mutagenesis of } \\
\text { Human Orai2 }\end{array}$ & Microsynth AG & $\begin{array}{l}\text { This study } \\
\text { (used to } \\
\text { generate } \\
\text { Human Orai2- } \\
\text { ANSGA) }\end{array}$ & $\begin{array}{l}\text { Forward primer: } \\
\text { 5'-CAT CCA CTT CTA CCG CTC CgC } \\
\text { Gaa tCG Cgg Cgc AAC GGA GCG } \\
\text { CCA CAA CC-3' }\end{array}$ \\
\hline $\begin{array}{l}\text { Sequenced- } \\
\text { based reagent }\end{array}$ & $\begin{array}{l}\text { R224S (ANSGA) } \\
\text { primers for } \\
\text { mutagenesis of } \\
\text { Human Orai2-ANRGA }\end{array}$ & Microsynth AG & This study & $\begin{array}{l}\text { Forward primer: } \\
\text { 5'-CTA CCG CTC CGC GAA TaG CGG } \\
\text { CGC AAC GGA GCG-3' }\end{array}$ \\
\hline $\begin{array}{l}\text { Sequenced- } \\
\text { based reagent }\end{array}$ & $\begin{array}{l}\text { L270A-V271N-H273G- } \\
\text { K274A (ANAGA) } \\
\text { primers for } \\
\text { mutagenesis of } \\
\text { Human Orai3 }\end{array}$ & Microsynth AG & $\begin{array}{l}\text { This study } \\
\text { (used to } \\
\text { generate } \\
\text { Human Orai3- } \\
\text { ANSGA) } \\
\end{array}$ & $\begin{array}{l}\text { Forward primer: } \\
\text { 5'-CTG CAT TTC TAC CGC TCC gcG } \\
\text { aat GCA ggg gcG ACA GAC CGC TAC } \\
\text { AAG C-3' }\end{array}$ \\
\hline $\begin{array}{l}\text { Sequenced- } \\
\text { based reagent }\end{array}$ & $\begin{array}{l}\text { A272S (ANSGA) } \\
\text { primers } \\
\text { for mutagenesis of } \\
\text { Human Orai3-ANAGA }\end{array}$ & Microsynth AG & This study & $\begin{array}{l}\text { Forward primer: } \\
\text { 5'-CTA CCG CTC CGC GAA TtC AGG } \\
\text { GGC GAC AGA CCG-3' }\end{array}$ \\
\hline $\begin{array}{l}\text { Sequenced- } \\
\text { based reagent }\end{array}$ & $\begin{array}{l}\text { F187C primers for } \\
\text { mutagenesis of } \\
\text { Human Orai1 }\end{array}$ & Microsynth AG & This study & $\begin{array}{l}\text { Forward primer: } \\
\text { 5'-CAT TGG CAC GCT GCT CTg CCT } \\
\text { AGC TGA GGT GGT GC-3' }\end{array}$ \\
\hline $\begin{array}{l}\text { Sequenced- } \\
\text { based reagent }\end{array}$ & $\begin{array}{l}\text { F161C primers for } \\
\text { mutagenesis of } \\
\text { Human Orai2 }\end{array}$ & Microsynth AG & This study & $\begin{array}{l}\text { Forward primer: } \\
\text { 5'-CTT GGC ATC CTA CTC TgC CTG } \\
\text { GCC GAG GTG GTG-3' }\end{array}$ \\
\hline $\begin{array}{l}\text { Sequenced- } \\
\text { based reagent }\end{array}$ & $\begin{array}{l}\text { F162C primers for } \\
\text { mutagenesis of } \\
\text { Human Orai3 }\end{array}$ & Microsynth AG & This study & $\begin{array}{l}\text { Forward primer: } \\
\text { 5'-CTG GGC ACC TTT CTC TgC CTT } \\
\text { GCT GAA GTT GTC-3' }\end{array}$ \\
\hline $\begin{array}{l}\text { Sequenced- } \\
\text { based reagent }\end{array}$ & $\begin{array}{l}\text { ANSGA primers for } \\
\text { mutagenesis of } \\
\text { Xenopus Orai } 1\end{array}$ & Microsynth AG & This study & $\begin{array}{l}\text { Forward primer: } \\
\text { 5'-GTT CAC TTT TAC AGA TCA gCA } \\
\text { aaC AGC ggT gCA ACT GAC CGT } \\
\text { CAG TTC C-3' }\end{array}$ \\
\hline $\begin{array}{l}\text { Sequenced- } \\
\text { based reagent }\end{array}$ & $\begin{array}{l}\text { ANSGA primers for } \\
\text { mutagenesis of } \\
\text { Drosophila Orai }\end{array}$ & Microsynth AG & This study & $\begin{array}{l}\text { Forward primer: } \\
\text { 5'-CAT TCA CTT CTA TCG CTC CgC } \\
\text { Gaa tTC Ggg Cgc ATA TGA GGT GAC } \\
\text { AGT CTC G-3' }\end{array}$ \\
\hline $\begin{array}{l}\text { Sequenced- } \\
\text { based reagent }\end{array}$ & $\begin{array}{l}\text { V76A primers for } \\
\text { mutagenesis of } \\
\text { Human Orai2-ANSGA }\end{array}$ & Microsynth AG & This study & $\begin{array}{l}\text { Forward primer: } \\
\text { 5'-CTC TCC GGC TTT GCC ATG GcG } \\
\text { GCC ATG GTG GAG GTG CAG-3' }\end{array}$ \\
\hline $\begin{array}{l}\text { Sequenced- } \\
\text { based reagent }\end{array}$ & $\begin{array}{l}\text { V77A primers for } \\
\text { mutagenesis of } \\
\text { Human Orai3-ANSGA }\end{array}$ & Microsynth AG & This study & $\begin{array}{l}\text { Forward primer: } \\
\text { 5'-CTC TCG GGC TTC GCC ATG GcG } \\
\text { GCC ATG GTG GAG GTG CAG-3' }\end{array}$ \\
\hline $\begin{array}{l}\text { Sequenced- } \\
\text { based reagent }\end{array}$ & $\begin{array}{l}\text { V174A primers for } \\
\text { mutagenesis of } \\
\text { Drosophila Orai- } \\
\text { ANSGA }\end{array}$ & Microsynth AG & This study & $\begin{array}{l}\text { Forward primer: } \\
\text { 5'-CGG ATT CGC CAT GGc cGC GAT } \\
\text { GGT GGA GG-3' }\end{array}$ \\
\hline $\begin{array}{l}\text { Sequenced- } \\
\text { based reagent }\end{array}$ & $\begin{array}{l}\text { H171Y primers for } \\
\text { mutagenesis of } \\
\text { Human Orai1/ } \\
\text { ANSGA/ V102C/ } \\
\text { H134A/ P245L/ F250C }\end{array}$ & Microsynth AG & This study & $\begin{array}{l}\text { Forward primer: } \\
\text { 5'-GAG CGC ATG CAC CGC tAC ATC } \\
\text { GAG CTG GCC TG-3' }\end{array}$ \\
\hline $\begin{array}{l}\text { Sequenced- } \\
\text { based reagent }\end{array}$ & $\begin{array}{l}\mathrm{H} 171 \mathrm{~A} \text { primers for } \\
\text { mutagenesis of } \\
\text { Human Orai1-ANSGA }\end{array}$ & Microsynth AG & This study & $\begin{array}{l}\text { Forward primer: } \\
\text { 5'-CAT GAG CGC ATG CAC CGC gcC } \\
\text { ATC GAG CTG GCC TGG GC-3' }\end{array}$ \\
\hline $\begin{array}{l}\text { Sequenced- } \\
\text { based reagent }\end{array}$ & $\begin{array}{l}\text { H171F primers for } \\
\text { mutagenesis of } \\
\text { Human Orai1-ANSGA }\end{array}$ & Microsynth AG & This study & $\begin{array}{l}\text { Forward primer: } \\
\text { 5'-CAT GAG CGC ATG CAC CGC ttC } \\
\text { ATC GAG CTG GCC TGG GC-3' }\end{array}$ \\
\hline
\end{tabular}


bioRxiv preprint doi: https://doi.org/10.1101/2021.10.26.465861; this version posted October 26, 2021. The copyright holder for this preprint (which was not certified by peer review) is the author/funder. All rights reserved. No reuse allowed without permission.

Augustynek et al.

Gating mechanisms of Orai calcium channels

\begin{tabular}{|c|c|c|c|c|}
\hline $\begin{array}{l}\text { Sequenced- } \\
\text { based reagent }\end{array}$ & $\begin{array}{l}\text { V102C primers for } \\
\text { mutagenesis of } \\
\text { Human Orai1/ H171Y- } \\
\text { ANSGA }\end{array}$ & Microsynth AG & This study & $\begin{array}{l}\text { Forward primer: } \\
\text { 5'-CTC CGG CTT CGC CAT Gtg cGC } \\
\text { AAT GGT GGA GGT GC-3' }\end{array}$ \\
\hline $\begin{array}{l}\text { Sequenced- } \\
\text { based reagent }\end{array}$ & $\begin{array}{l}\text { A } 175 \mathrm{C} \text { primers for } \\
\text { mutagenesis of } \\
\text { Human Orai1-ANSGA }\end{array}$ & Microsynth AG & This study & $\begin{array}{l}\text { Forward primer: } \\
\text { 5'-CAC CGC CAC ATC GAG CTG tgC } \\
\text { TGG GCC TTC TCC ACC G-3' }\end{array}$ \\
\hline $\begin{array}{l}\text { Sequenced- } \\
\text { based reagent }\end{array}$ & $\begin{array}{l}\text { Y258C primers for } \\
\text { mutagenesis of } \\
\text { Human Orai1-ANSGA/ } \\
\text { A175C-ANSGA }\end{array}$ & Microsynth AG & This study & $\begin{array}{l}\text { Forward primer: } \\
\text { 5'-CTT CGC CGT CCA CTT CTg CCG } \\
\text { CTC AGC GAA TAG CG-3' }\end{array}$ \\
\hline $\begin{array}{l}\text { Sequenced- } \\
\text { based reagent }\end{array}$ & $\begin{array}{l}\text { L174D primers for } \\
\text { mutagenesis of } \\
\text { Human Orai1-ANSGA }\end{array}$ & Microsynth AG & This study & $\begin{array}{l}\text { Forward primer: } \\
\text { 5'-CAC CGC CAC ATC GAG gac GCC } \\
\text { TGG GCC TTC TCC-3' }\end{array}$ \\
\hline $\begin{array}{l}\text { Sequenced- } \\
\text { based reagent }\end{array}$ & $\begin{array}{l}\text { F178A primers for } \\
\text { mutagenesis of } \\
\text { Human Orai1-ANSGA }\end{array}$ & Microsynth AG & This study & $\begin{array}{l}\text { Forward primer: } \\
\text { 5'-GAG CTG GCC TGG GCC gcC TCC } \\
\text { ACC GTC ATT GG-3' }\end{array}$ \\
\hline $\begin{array}{l}\text { Sequenced- } \\
\text { based reagent }\end{array}$ & $\begin{array}{l}\text { F257A primers for } \\
\text { mutagenesis of } \\
\text { Human Orai1-ANSGA }\end{array}$ & Microsynth AG & This study & $\begin{array}{l}\text { Forward primer: } \\
\text { 5'-CGT CTT CGC CGT CCA Cgc CTA } \\
\text { CCG CTC AGC GAA TAG-3' }\end{array}$ \\
\hline $\begin{array}{l}\text { Sequenced- } \\
\text { based reagent }\end{array}$ & $\begin{array}{l}\text { F250C primers for } \\
\text { mutagenesis of } \\
\text { Human Orai1 }\end{array}$ & Microsynth AG & This study & $\begin{array}{l}\text { Forward primer: } \\
\text { 5'-CCC TTC GGC CTG ATC TgT ATC } \\
\text { GTC TTC GCC GTC-3' }\end{array}$ \\
\hline $\begin{array}{l}\text { Sequenced- } \\
\text { based reagent }\end{array}$ & $\begin{array}{l}\text { P144R-Y145H primers } \\
\text { for mutagenesis of } \\
\text { Human Orai2-ANSGA }\end{array}$ & Microsynth AG & This study & $\begin{array}{l}\text { Forward primer: } \\
\text { 5'-CGC ATG AGC GCA TGC ATC gCc } \\
\text { ACA TCG AGC TGG CCT GGG-3' }\end{array}$ \\
\hline $\begin{array}{l}\text { Sequenced- } \\
\text { based reagent }\end{array}$ & $\begin{array}{l}\text { Y146H primers for } \\
\text { mutagenesis of } \\
\text { Human Orai3-ANSGA }\end{array}$ & Microsynth AG & This study & $\begin{array}{l}\text { Forward primer: } \\
\text { 5'-CCA GAG ACT GCA CCG CCA CGT } \\
\text { GGA GCT GGC CTG-3' }\end{array}$ \\
\hline $\begin{array}{l}\text { Sequenced- } \\
\text { based reagent }\end{array}$ & $\begin{array}{l}\text { T266stop }(\Delta \mathrm{CT}) \\
\text { primers for } \\
\text { mutagenesis of } \\
\text { Human Orai1-ANSGA/ } \\
\text { H171Y-ANSGA }\end{array}$ & Microsynth AG & This study & $\begin{array}{l}\text { Forward primer: } \\
\text { 5'-CAG CGA ATA GCG GTG CGt gaG } \\
\text { ACC GAC AGT TCC AGG AG-3' }\end{array}$ \\
\hline $\begin{array}{l}\text { Sequenced- } \\
\text { based reagent }\end{array}$ & $\begin{array}{l}\text { I251V primers for } \\
\text { mutagenesis of } \\
\text { Human Orai1-ANSGA }\end{array}$ & Microsynth AG & This study & $\begin{array}{l}\text { Forward primer: } \\
\text { 5'-CCC TTC GGC CTG ATC TTT gTC } \\
\text { GTC TTC GCC GTC CAC-3' }\end{array}$ \\
\hline $\begin{array}{l}\text { Sequenced- } \\
\text { based reagent }\end{array}$ & $\begin{array}{l}\text { F246V primers for } \\
\text { mutagenesis of } \\
\text { Human Orai1/ } \\
\text { ANSGA/ V102C/ } \\
\text { H134A/ ANSGA- } \triangle C T\end{array}$ & Microsynth AG & This study & $\begin{array}{l}\text { Forward primer: } \\
\text { 5'-CCA CCA TCA TGG TGC CCg TCG } \\
\text { GCC TGA TCT TTA TC-3' }\end{array}$ \\
\hline $\begin{array}{l}\text { Sequenced- } \\
\text { based reagent }\end{array}$ & $\begin{array}{l}\text { F246C primers for } \\
\text { mutagenesis of } \\
\text { Human Orai1-ANSGA }\end{array}$ & Microsynth AG & This study & $\begin{array}{l}\text { Forward primer: } \\
\text { 5'-CAC CAT CAT GGT GCC CTg CGG } \\
\text { CCT GAT CTT TAT CG-3' }\end{array}$ \\
\hline $\begin{array}{l}\text { Sequenced- } \\
\text { based reagent }\end{array}$ & $\begin{array}{l}\text { F246Y primers for } \\
\text { mutagenesis of } \\
\text { Human Orai1-ANSGA }\end{array}$ & Microsynth AG & This study & $\begin{array}{l}\text { Forward primer: } \\
\text { 5'-CCA CCA TCA TGG TGC CCT aCG } \\
\text { GCC TGA TCT TTA TCG TC-3' }\end{array}$ \\
\hline $\begin{array}{l}\text { Sequenced- } \\
\text { based reagent }\end{array}$ & $\begin{array}{l}\text { A254T-V255I primers } \\
\text { for mutagenesis of } \\
\text { Human Orai1-ANSGA }\end{array}$ & Microsynth AG & This study & $\begin{array}{l}\text { Forward primer: } \\
\text { 5'-GCC TGA TCT TTA TCG TCT TCa } \\
\text { CCa TCC ACT TCT ACC GCT CAG } \\
\text { CG-3' }\end{array}$ \\
\hline $\begin{array}{l}\text { Sequenced- } \\
\text { based reagent }\end{array}$ & $\begin{array}{l}\text { F246V-I251V primers } \\
\text { for mutagenesis of } \\
\text { Human Orai1-ANSGA }\end{array}$ & Microsynth AG & $\begin{array}{l}\text { This study } \\
\text { (used to } \\
\text { generate } \\
\text { Human Orai1- } \\
\text { F246V-I251V- } \\
\text { A254T-V255I- } \\
\text { ANSGA) }\end{array}$ & $\begin{array}{l}\text { Forward primer: } \\
\text { 5'-CAC CAT CAT GGT GCC CgT CGG } \\
\text { CCT GAT CTT TgT CGT CTT CGC } \\
\text { CGT CCA C-3' }\end{array}$ \\
\hline
\end{tabular}


bioRxiv preprint doi: https://doi.org/10.1101/2021.10.26.465861; this version posted October 26, 2021. The copyright holder for this preprint (which was not certified by peer review) is the author/funder. All rights reserved. No reuse allowed without permission.

Augustynek et al.

Gating mechanisms of Orai calcium channels

\begin{tabular}{|c|c|c|c|c|}
\hline $\begin{array}{l}\text { Sequenced- } \\
\text { based reagent }\end{array}$ & $\begin{array}{l}\text { A254T-V255I primers } \\
\text { for mutagenesis of } \\
\text { Human Orai1-F246V- } \\
\text { I251V-ANSGA }\end{array}$ & Microsynth AG & This study & $\begin{array}{l}\text { Forward primer: } \\
\text { 5'-GCC TGA TCT TTG TCG TCT TCa } \\
\text { CCa TCC ACT TCT ACC GCT CAG } \\
\text { CG-3' }\end{array}$ \\
\hline $\begin{array}{l}\text { Sequenced- } \\
\text { based reagent }\end{array}$ & $\begin{array}{l}\text { I249V-V252A-V255L } \\
\text { primers for } \\
\text { mutagenesis of } \\
\text { Human Orai1-ANSGA }\end{array}$ & Microsynth AG & This study & $\begin{array}{l}\text { Forward primer: } \\
\text { 5'-GGT GCC CTT CGG CCT GgT CTT } \\
\text { TAT CGC CTT CGC CCT CCA CTT } \\
\text { CTA CCG CTC-3' }\end{array}$ \\
\hline $\begin{array}{l}\text { Sequenced- } \\
\text { based reagent }\end{array}$ & $\begin{array}{l}\text { F246V-I251V primers } \\
\text { for mutagenesis of } \\
\text { Human Orai1-I249V- } \\
\text { V252A-V255L-ANSGA }\end{array}$ & Microsynth AG & This study & $\begin{array}{l}\text { Forward primer: } \\
\text { 5'-CAC CAT CAT GGT GCC CgT CGG } \\
\text { CCT GGT CTT TgT CGC CTT CGC } \\
\text { CCT CCA C-3' }\end{array}$ \\
\hline $\begin{array}{l}\text { Sequenced- } \\
\text { based reagent }\end{array}$ & $\begin{array}{l}1249 \mathrm{~V} \text { primers for } \\
\text { mutagenesis of } \\
\text { Human Orai1-ANSGA }\end{array}$ & Microsynth AG & This study & $\begin{array}{l}\text { Forward primer: } \\
\text { 5'-CAT GGT GCC CTT CGG CCT GgT } \\
\text { CTT TAT CGT CTT CGC CGT C-3' }\end{array}$ \\
\hline $\begin{array}{l}\text { Sequenced- } \\
\text { based reagent }\end{array}$ & $\begin{array}{l}\text { V252A primers for } \\
\text { mutagenesis of } \\
\text { Human Orai1-ANSGA }\end{array}$ & Microsynth AG & This study & $\begin{array}{l}\text { Forward primer: } \\
\text { 5'-CGG CCT GAT CTT TAT CGc CTT } \\
\text { CGC CGT CCA CTT C-3' }\end{array}$ \\
\hline $\begin{array}{l}\text { Sequenced- } \\
\text { based reagent }\end{array}$ & $\begin{array}{l}\text { V255L primers for } \\
\text { mutagenesis of } \\
\text { Human Orai1-ANSGA }\end{array}$ & Microsynth AG & This study & $\begin{array}{l}\text { Forward primer: } \\
\text { 5'-GAT CTT TAT CGT CTT CGC CcT } \\
\text { CCA CTT CTA CCG CTC AGC-3' }\end{array}$ \\
\hline $\begin{array}{l}\text { Sequenced- } \\
\text { based reagent }\end{array}$ & $\begin{array}{l}\text { V255L primers for } \\
\text { mutagenesis of } \\
\text { Human Orai1-I249V- } \\
\text { ANSGA }\end{array}$ & Microsynth AG & This study & $\begin{array}{l}\text { Forward primer: } \\
\text { 5'-CGG CCT GGT CTT TAT CGT CTT } \\
\text { CGC CCT CCA CTT C-3' }\end{array}$ \\
\hline $\begin{array}{l}\text { Sequenced- } \\
\text { based reagent }\end{array}$ & $\begin{array}{l}\text { P206C primers for } \\
\text { mutagenesis of } \\
\text { Human Orai2 }\end{array}$ & Microsynth AG & This study & $\begin{array}{l}\text { Forward primer: } \\
\text { 5'-CCA CCA TCA TCA TGG TGt gCG } \\
\text { TGG GCC TCA TCT TCG-3' }\end{array}$ \\
\hline $\begin{array}{l}\text { Sequenced- } \\
\text { based reagent }\end{array}$ & $\begin{array}{l}\text { F211C primers for } \\
\text { mutagenesis of } \\
\text { Human Orai2 }\end{array}$ & Microsynth AG & This study & $\begin{array}{l}\text { Forward primer: } \\
\text { 5'-CCC GTG GGC CTC ATC TgC GTG } \\
\text { GTC TTC ACC ATC-3' }\end{array}$ \\
\hline $\begin{array}{l}\text { Sequenced- } \\
\text { based reagent }\end{array}$ & $\begin{array}{l}\text { P254C primers for } \\
\text { mutagenesis of } \\
\text { Human Orai3 }\end{array}$ & Microsynth AG & This study & $\begin{array}{l}\text { Forward primer: } \\
\text { 5'-CCA CAG CCA TCA TGG Tat gCG } \\
\text { TGG GGC TCG TGT TTG-3' }\end{array}$ \\
\hline $\begin{array}{l}\text { Sequenced- } \\
\text { based reagent }\end{array}$ & $\begin{array}{l}\text { F259C primers for } \\
\text { mutagenesis of } \\
\text { Human Orai3 }\end{array}$ & Microsynth AG & This study & $\begin{array}{l}\text { Forward primer: } \\
\text { 5'-CCC GTG GGG CTC GTG TgT GTG } \\
\text { GCC TTT GCC CTG-3' }\end{array}$ \\
\hline $\begin{array}{l}\text { Sequenced- } \\
\text { based reagent }\end{array}$ & $\begin{array}{l}\text { F246V primers for } \\
\text { mutagenesis of } \\
\text { Human Orai1-P245L }\end{array}$ & Microsynth AG & This study & $\begin{array}{l}\text { Forward primer: } \\
\text { 5'-CCA CCA TCA TGG TGC TCg TCG } \\
\text { GCC TGA TCT TTA TC-3' }\end{array}$ \\
\hline $\begin{array}{l}\text { Sequenced- } \\
\text { based reagent }\end{array}$ & $\begin{array}{l}\text { F246V primers for } \\
\text { mutagenesis of } \\
\text { Human Orai1-F250C }\end{array}$ & Microsynth AG & This study & $\begin{array}{l}\text { Forward primer: } \\
\text { 5'-CCA CCA TCA TGG TGC CCg TCG } \\
\text { GCC TGA TCT GTA TC-3' }\end{array}$ \\
\hline $\begin{array}{l}\text { Sequenced- } \\
\text { based reagent }\end{array}$ & $\begin{array}{l}\text { V207F-V212I primers } \\
\text { for mutagenesis of } \\
\text { Human Orai2-P144R- } \\
\text { Y145H-ANSGA }\end{array}$ & Microsynth AG & This study & $\begin{array}{l}\text { Forward primer: } \\
\text { 5'-CCA TCA TCA TGG TGC CCt TCG } \\
\text { GCC TCA TCT TCa TCG TCT TCA } \\
\text { CCA TCC ACT TC-3' }\end{array}$ \\
\hline $\begin{array}{l}\text { Sequenced- } \\
\text { based reagent }\end{array}$ & $\begin{array}{l}\text { V255F-V260I-A261V } \\
\text { primers for } \\
\text { mutagenesis of } \\
\text { Human Orai3-Y146H- } \\
\text { ANSGA }\end{array}$ & Microsynth AG & This study & $\begin{array}{l}\text { Forward primer: } \\
\text { 5'-CAG CCA TCA TGG TAC CCt TCG } \\
\text { GGC TCG TGT TTa TCG tCT TTG } \\
\text { CCC TGC ATT TCT-3' }\end{array}$ \\
\hline $\begin{array}{l}\text { Sequenced- } \\
\text { based reagent }\end{array}$ & $\begin{array}{l}\text { Primers to remove C- } \\
\text { terminal eGFP tag } \\
\text { from Mouse WT Orai1 } \\
\text { in peGFP-N1 }\end{array}$ & Microsynth AG & This study & $\begin{array}{l}\text { Forward primer: } \\
\text { 5'-GGG CAC CCA CTA TGC Cta GGA } \\
\text { TCC ACC GGT CGC C-3' }\end{array}$ \\
\hline $\begin{array}{l}\text { Sequenced- } \\
\text { based reagent }\end{array}$ & $\begin{array}{l}\text { L264A-V265N-H267G- } \\
\text { K268A (ANSGA) } \\
\text { primers for } \\
\text { mutagenesis of Mouse } \\
\text { Orai1 }\end{array}$ & Microsynth AG & This study & $\begin{array}{l}\text { Forward primer: } \\
\text { 5'-GTT CAC TTC TAC CGt TCC gcG } \\
\text { aat AGC ggT gcG ACG GAC CGG } \\
\text { CAG TTC CAG-3' }\end{array}$ \\
\hline
\end{tabular}


bioRxiv preprint doi: https://doi.org/10.1101/2021.10.26.465861; this version posted October 26, 2021. The copyright holder for this preprint (which was not certified by peer review) is the author/funder. All rights reserved. No reuse allowed without permission.

Augustynek et al.

Gating mechanisms of Orai calcium channels

\begin{tabular}{|c|c|c|c|c|}
\hline $\begin{array}{l}\text { Sequenced- } \\
\text { based reagent }\end{array}$ & $\begin{array}{l}\text { C249F primers for } \\
\text { mutagenesis of mouse } \\
\text { Orai1 WT and ANSGA }\end{array}$ & Microsynth AG & This study & $\begin{array}{l}\text { Forward primer: } \\
\text { 5'-CCG CCA TCA TGG TTC CCT tTG } \\
\text { GCC TGG TTT TTA TCG TC-3' }\end{array}$ \\
\hline $\begin{array}{l}\text { Sequenced- } \\
\text { based reagent }\end{array}$ & $\begin{array}{l}\text { Human STIM1 gRNA1 } \\
\text { primer for cloning in } \\
\text { CRISPR plasmid }\end{array}$ & Microsynth AG & This study & $\begin{array}{l}\text { Forward primer: } \\
\text { 5'-CAC CGT TCT GTG CCC GCG } \\
\text { GAG ACT C-3' }\end{array}$ \\
\hline $\begin{array}{l}\text { Sequenced- } \\
\text { based reagent }\end{array}$ & $\begin{array}{l}\text { Human STIM1 gRNA1 } \\
\text { primer for cloning in } \\
\text { CRISPR plasmid }\end{array}$ & Microsynth AG & This study & $\begin{array}{l}\text { Reverse primer: } \\
\text { 5'-AAA CGA GTC TCC GCG GGC } \\
\text { ACA GAA C-3' }\end{array}$ \\
\hline $\begin{array}{l}\text { Sequenced- } \\
\text { based reagent }\end{array}$ & $\begin{array}{l}\text { Human STIM1 gRNA2 } \\
\text { primer for cloning in } \\
\text { CRISPR plasmid }\end{array}$ & Microsynth AG & This study & $\begin{array}{l}\text { Forward primer: } \\
\text { 5'-CAC CGT ATG CGT CCG TCT TGC } \\
\text { CCT G-3' }\end{array}$ \\
\hline $\begin{array}{l}\text { Sequenced- } \\
\text { based reagent }\end{array}$ & $\begin{array}{l}\text { Human STIM1 gRNA2 } \\
\text { primer for cloning in } \\
\text { CRISPR plasmid }\end{array}$ & Microsynth AG & This study & $\begin{array}{l}\text { Reverse primer: } \\
\text { 5'-AAA CCA GGG CAA GAC GGA } \\
\text { CGC ATA C-3' }\end{array}$ \\
\hline $\begin{array}{l}\text { Sequenced- } \\
\text { based reagent }\end{array}$ & $\begin{array}{l}\text { Human STIM2 gRNA1 } \\
\text { primer for cloning in } \\
\text { CRISPR plasmid }\end{array}$ & Microsynth AG & This study & $\begin{array}{l}\text { Forward primer: } \\
\text { 5'-CAC CGC GGA ACC AAT GAA } \\
\text { CGC AGC C-3' }\end{array}$ \\
\hline $\begin{array}{l}\text { Sequenced- } \\
\text { based reagent }\end{array}$ & $\begin{array}{l}\text { Human STIM2 gRNA1 } \\
\text { primer for cloning in } \\
\text { CRISPR plasmid }\end{array}$ & Microsynth AG & This study & $\begin{array}{l}\text { Reverse primer: } \\
\text { 5'-AAA CGG CTG CGT TCA TTG GTT } \\
\text { CCG C-3' }\end{array}$ \\
\hline $\begin{array}{l}\text { Sequenced- } \\
\text { based reagent }\end{array}$ & $\begin{array}{l}\text { Human STIM2 gRNA2 } \\
\text { primer for cloning in } \\
\text { CRISPR plasmid }\end{array}$ & Microsynth AG & This study & $\begin{array}{l}\text { Forward primer: } \\
\text { 5'-CAC CGC TGG TAG CCG GAG } \\
\text { CGG CGG A-3' }\end{array}$ \\
\hline $\begin{array}{l}\text { Sequenced- } \\
\text { based reagent }\end{array}$ & $\begin{array}{l}\text { Human STIM2 gRNA2 } \\
\text { primer for cloning in } \\
\text { CRISPR plasmid }\end{array}$ & Microsynth AG & This study & $\begin{array}{l}\text { Reverse primer: } \\
\text { 5'-AAA CTC CGC CGC TCC GGC TAC } \\
\text { CAG C-3' }\end{array}$ \\
\hline Antibody & $\begin{array}{l}\text { Guinea pig polyclonal } \\
\text { for Human STIM1 }\end{array}$ & $\begin{array}{l}\text { (Ercan et al., } \\
\text { 2012) }\end{array}$ & & \\
\hline Antibody & $\begin{array}{l}\text { Goat anti-guinea pig } \\
\text { secondary antibody }\end{array}$ & $\begin{array}{l}\text { Jackson } \\
\text { Immuno } \\
\text { Research }\end{array}$ & $\begin{array}{l}\text { Cat\# 106-035- } \\
003\end{array}$ & \\
\hline Antibody & $\begin{array}{l}\text { Rabbit polyclonal for } \\
\text { Human STIM2 }\end{array}$ & Cell Signaling & Cat\# 4917S & \\
\hline Antibody & $\begin{array}{l}\text { Goat anti-rabbit } \\
\text { secondary }\end{array}$ & Promega & Cat\# W401B & \\
\hline Antibody & $\begin{array}{l}\text { Mouse monoclonal for } \\
\text { Tubulin }\end{array}$ & Sigma-Aldrich & Cat\# T9028 & \\
\hline Antibody & $\begin{array}{l}\text { Goat anti-mouse } \\
\text { secondary }\end{array}$ & Bio-Rad & Cat\# 172-1011 & \\
\hline $\begin{array}{l}\text { Software, } \\
\text { algorithm }\end{array}$ & ScreenWorks 3.1.1.8 & $\begin{array}{l}\text { Molecular } \\
\text { Devices, LLC }\end{array}$ & & \\
\hline $\begin{array}{l}\text { Software, } \\
\text { algorithm }\end{array}$ & GraphPad Prism 9.1.0 & $\begin{array}{l}\text { GraphPad } \\
\text { Software, LLC }\end{array}$ & & \\
\hline $\begin{array}{l}\text { Software, } \\
\text { algorithm }\end{array}$ & PyMol & Schrödinger & & \\
\hline $\begin{array}{l}\text { Software, } \\
\text { algorithm }\end{array}$ & PatchMaster & HEKA Elektronik & & \\
\hline
\end{tabular}

